\title{
Label-Free Biosensing Using Gold Coated Polysilicon Gate BioFETs
}

\author{
by \\ Ehsan Ghias-Begloo, B. Eng.
}

\author{
A Thesis Submitted to \\ the Faculty of Graduate Studies and Research \\ in Partial Fulfillment of the Requirements for the Degree of \\ Master of Applied Science \\ in Electrical Engineering \\ Ottawa-Carleton Institute for \\ Electrical and Computer Engineering
}

Department of Electronics

Carleton University

Ottawa, Ontario, Canada

May 2011

Copyright (C) 2011

Ehsan Ghias-Begloo 
Library and Archives

Canada

Published Heritage Branch

395 Wellington Street Ottawa ON K1A ON4 Canada
Bibliothèque et

Archives Canada

Direction du

Patrimoine de l'édition

395, rue Wellington

Ottawa ON K1A ON4

Canada
Your file Votre référence

ISBN: 978-0-494-81678-3

Our file Notre référence

ISBN: 978-0-494-81678-3
NOTICE:

The author has granted a nonexclusive license allowing Library and Archives Canada to reproduce, publish, archive, preserve, conserve, communicate to the public by telecommunication or on the Internet, loan, distribute and sell theses worldwide, for commercial or noncommercial purposes, in microform, paper, electronic and/or any other formats.

The author retains copyright ownership and moral rights in this thesis. Neither the thesis nor substantial extracts from it may be printed or otherwise reproduced without the author's permission.
AVIS:

L'auteur a accordé une licence non exclusive permettant à la Bibliothèque et Archives Canada de reproduire, publier, archiver, sauvegarder, conserver, transmettre au public par télécommunication ou par l'Internet, prêter, distribuer et vendre des thèses partout dans le monde, à des fins commerciales ou autres, sur support microforme, papier, électronique et/ou autres formats.

L'auteur conserve la propriété du droit d'auteur et des droits moraux qui protège cette thèse. $\mathrm{Ni}$ la thèse ni des extraits substantiels de celle-ci ne doivent être imprimés ou autrement reproduits sans son autorisation.
In compliance with the Canadian Privacy Act some supporting forms may have been removed from this thesis.

While these forms may be included in the document page count, their removal does not represent any loss of content from the thesis.
Conformément à la loi canadienne sur la protection de la vie privée, quelques formulaires secondaires ont été enlevés de cette thèse.

Bien que ces formulaires aient inclus dans la pagination, il n'y aura aucun contenu manquant.

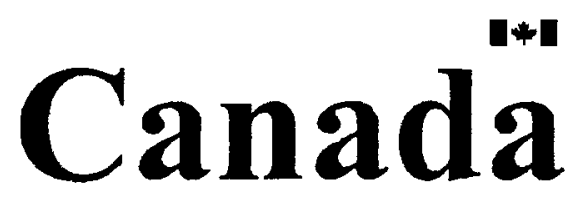


The undersigned recommend to the Faculty of Graduate and Postdoctoral Affairs acceptance of the thesis

\title{
Label-Free Biosensing Using Gold Coated Polysilicon Gate BioFETs
}

\author{
Submitted by \\ Ehsan Ghıs-Begloo, B Eng
}

In partial fulfillment of the requirements for the degree of

Master's of Applied Science

in Electrical Engineerıng

Professor Garry Tarr, Co-Supervisor

Professor Niall Tait, Co-Supervisor

Adjunct Professor Dolf Landheer, Co-Supervisor

\author{
Professor Q1-Jun Zhang \\ Chair, Department of Electronics
}

Ottawa-Carleton Institute for Electrıcal and Computer Engıneerıng

Carleton University

Department of Electronics

2011 


\section{Abstract}

Label-free electronic detection of thiol-terminated DNA molecules is reported, employing an array of chromium-gold coated bioFETs fabricated based on silicon $<111>$ crystal wafers. BioFET gates with polysilicon as an intermediate layer between the chromium-gold surface and silicon dioxide dielectric were compared to etched polysilicon gates. Enhanced electrochemical properties of gold surfaces are expected to support higher sensitivity than previously reported bioFETs. Functionalization and hybridization of thiol-terminated DNA strands were effectively performed and successful detection was observed on multiple devices. Although similar behaviour was observed in most devices in each chip, non-uniformity in the post-processed devices resulted in inconsistent results. Improvements in post-processing steps, surface chemistry, and electrolyte are expected to enhance the sensitivity, consistency, selectivity, and stability of detection. 


\section{Acknowledgments}

I would like to exclusively thank:

Dr. Garry Tarr and Dr. Niall Tait for their patience, motivation and enlightening guidance throughout my academic career.

Rob Vandusen and Carol Adams for their time and expertise.

Dr. Dolf Landheer for his supervision and guidance for the measurements.

Dr. Weihong Jiang for her efforts on the post-processing steps.

Dr. Maria DeRosa and Tariq Francis for providing the DNA solutions.

David Blair for his assistance in performing the measurements.

My especial gratitude to my parents who have supported me and been my greatest encouragement to succeed throughout my prolonged academic career. 


\section{Table of Contents}

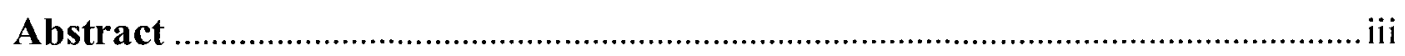

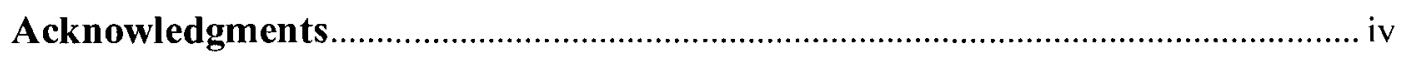

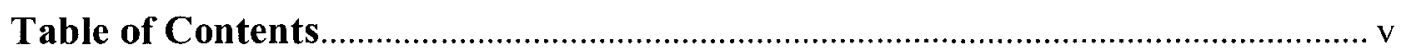

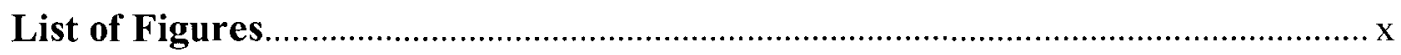

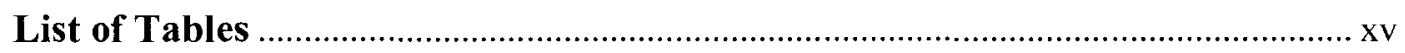

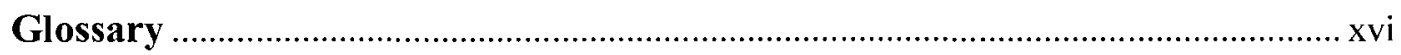

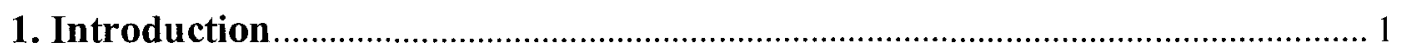

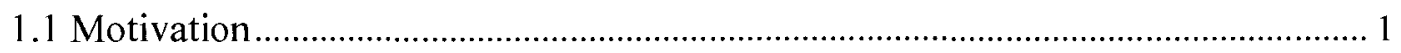

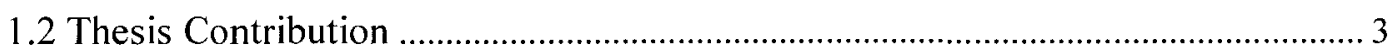

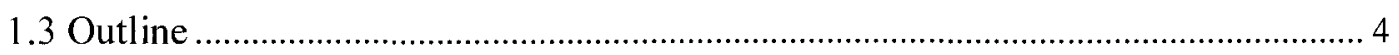

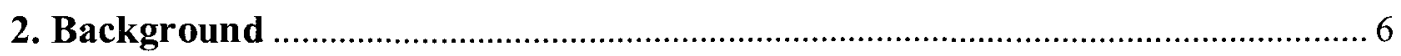

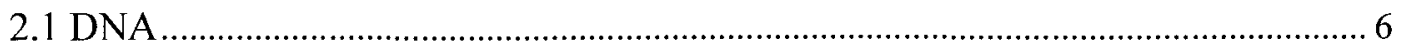

2.1.1 Bases of Nucleic Acids .....................................................................

2.1.2 Sugars Present at Nucleic Acids ...................................................... 


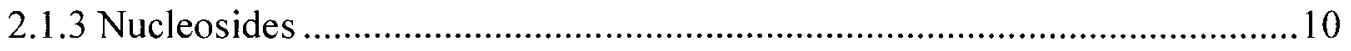

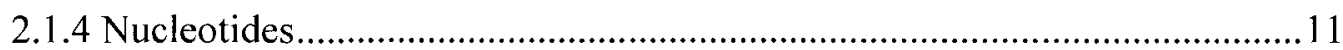

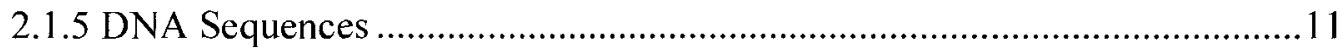

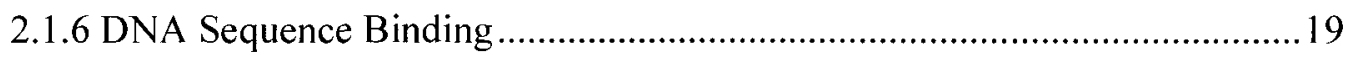

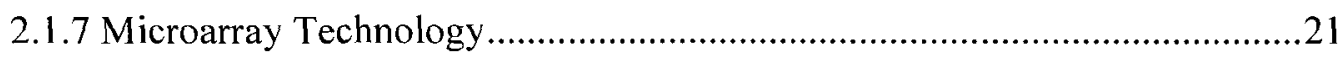

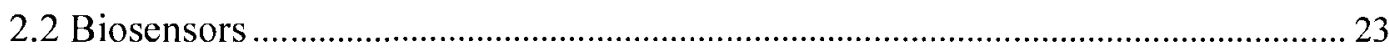

2.2.1 Label-based Sensing Methods...............................................................24

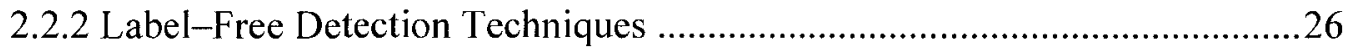

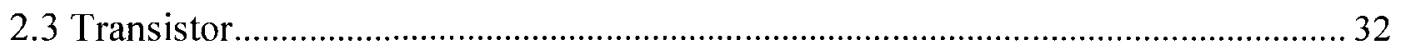

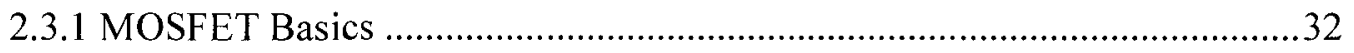

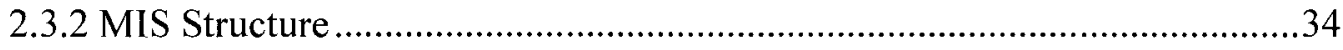

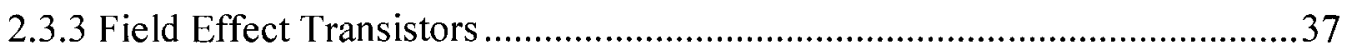

2.4 Ion Sensitive Field Effect Transistors ............................................................... 43

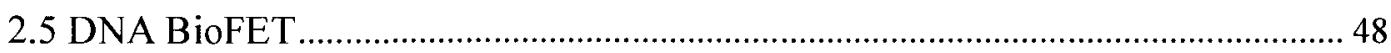

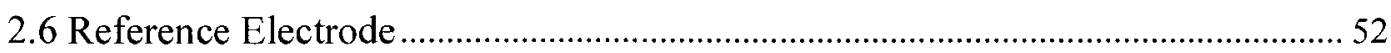




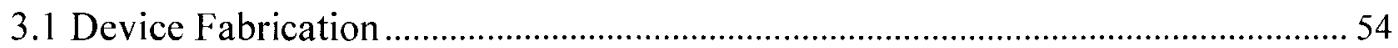

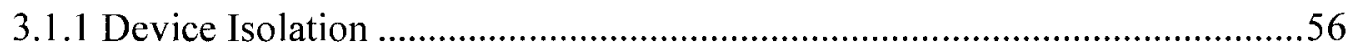

3.1.2 Gate and Junctions Formation................................................................5

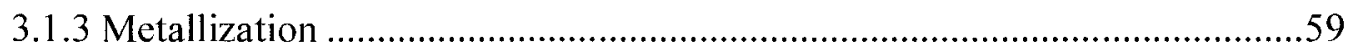

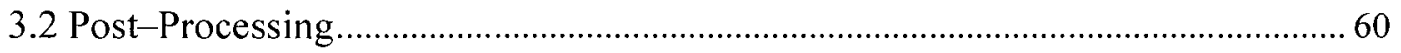

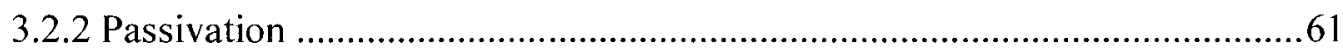

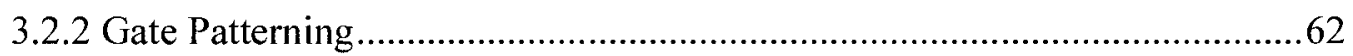

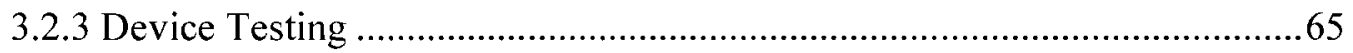

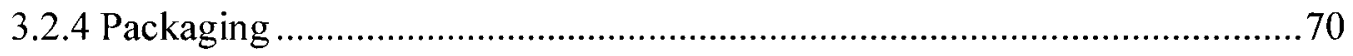

4. Measurement Setup and Testing.................................................................................. 72

4.1 Measurement Setup .........................................................................................

4.1.1 Electrical Readings ...................................................................................

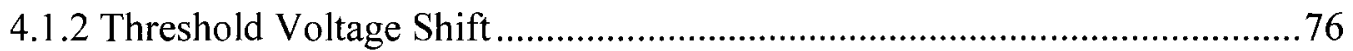

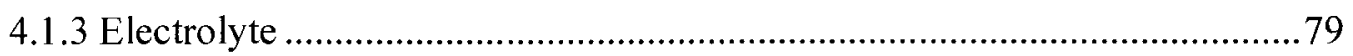

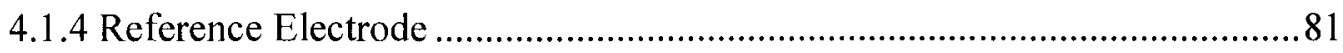




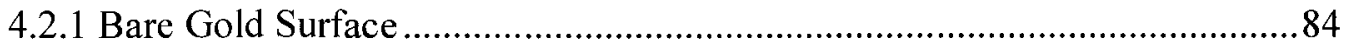

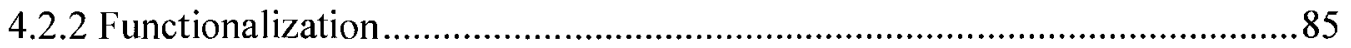

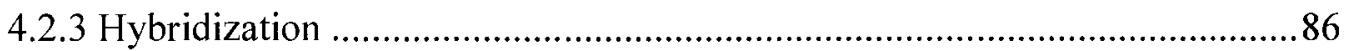

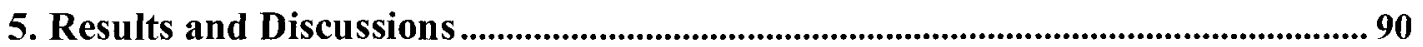

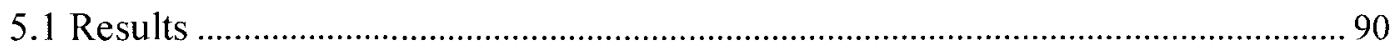

5.1.1 Gold-Chromium-Oxide Gate Devices ..............................................90

5.1.2 Gold-Chromium-Poly-Oxide Gate Devices........................................ 100

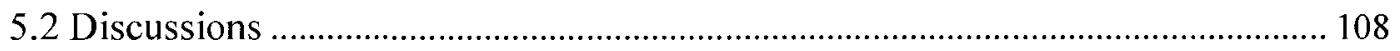

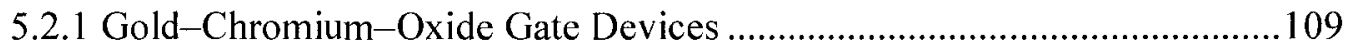

5.2.2 Gold-Chromium-Poly-Oxide Gate Devices....................................112

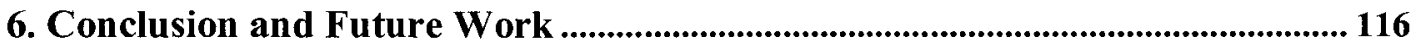

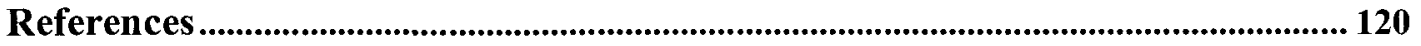

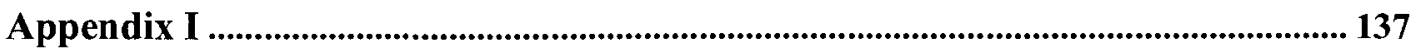

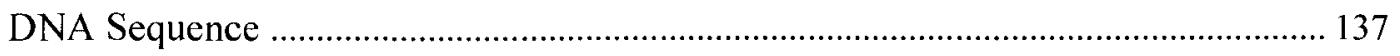

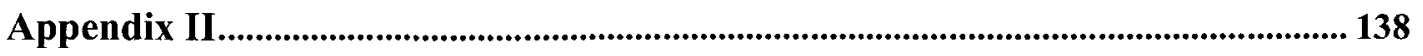




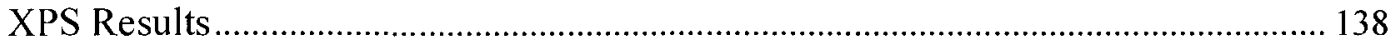




\section{List of Figures}

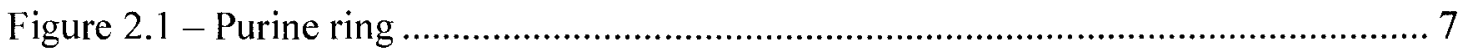

Figure 2.2 - Purine bases: (a) Adenine; and (b) Guanine........................................ 8

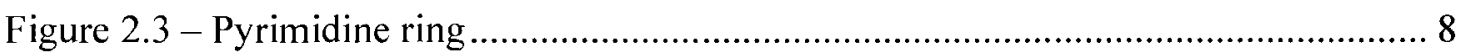

Figure 2.4 - Pyrimidines bases: (a) Cytosine; (b) Thymine; and (c) Uracil.................... 9

Figure 2.5 - (a) $\beta$-D-ribose; and (b) $\beta-D-2-$ deoxyribose, sugars present at nucleic acids.

Figure 2.6 - Nucleosides: Deoxyadenosine; (b) Deoxyguanosine; (c) Deoxycytidine; and

(d) Deoxythymidine.

Figure 2.7 - Nucleotides: Phosphate deoxyadenosine; (b) Phosphate deoxyguanosine; (c) Phosphate deoxycytidine; and (d) Phosphate deoxythymidine. 11

Figure 2.8 - A Simple polynucleotide present in DNA ............................................ 13

Figure 2.9 - Watson-Crick base pairs: (a) Adenine - Thymine pair; and (b) Guanine Cytosine pair. 14

Figure 2.10 - A 4-mer Double-Stranded DNA sequence (ACGT).......................... 15

Figure 2.11 - A 15-mer Double-Stranded DNA sequence (GTAGTCGTCTAGGCA). 16 Figure 2.12 - A simple four terminal MOSFET. 33

Figure 2.13 - Charge distribution in the conducting channel region of a MOSFET ....... 34

Figure 2.14 - One dimensional view of a MIS structure ............................................ 35 
Figure 2.15 - The Energy bands of a p-type MIS structure as a function of applied voltage, $\mathrm{V}_{\mathrm{GB}}$, in (a) Equilibrium; (b) Accumulation; (c) Depletion; (d) Deep Depletion;

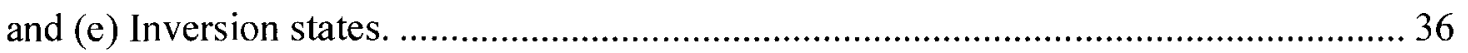
Figure 2.16 - A p-FET in different states: (a) Sub-Threshold, $V_{\mathrm{GS}}<\mathrm{V}_{\mathrm{T}}$; (b) $\mathrm{V}_{\mathrm{GS}}>\mathrm{V}_{\mathrm{T}}$, $\mathrm{V}_{\mathrm{DS}}<\mathrm{V}_{\mathrm{T}}$; (c) $\mathrm{V}_{\mathrm{GS}}>\mathrm{V}_{\mathrm{T}}, \mathrm{V}_{\mathrm{DS}}<\mathrm{V}_{\mathrm{GS}}-\mathrm{V}_{\mathrm{T}}$; (d) $\mathrm{V}_{\mathrm{GS}}>\mathrm{V}_{\mathrm{T}}, \mathrm{V}_{\mathrm{DS}}>\mathrm{V}_{\mathrm{GS}}-\mathrm{V}_{\mathrm{T}}, \mathrm{V}_{\mathrm{DS}}>\mathrm{V}_{\mathrm{DSsat}} .38$

Figure 2.17 - A 3-D view of a FET. 41

Figure 2.18 - The $\mathrm{I}_{\mathrm{DS}} v s . \mathrm{V}_{\mathrm{DS}}$ plot showing the unsaturated and saturated regions for different values of $V_{G S}$ and $\alpha=1$

Figure 2.19 - A general schematic of an ISFET 44

Figure $2.20-\mathrm{I}_{\mathrm{DS}}$ current $v s . \mathrm{V}_{\mathrm{DS}}$ in a MOSFET (solid) compared to the $\mathrm{I}_{\mathrm{DS}}$ of an ISFET with $1 \mathrm{mM}$ (dashed) and $0.1 \mathrm{M}$ (long dashed) electrolytes. $\mathrm{V}_{\mathrm{GS}}$ in the ISFET is applied through a reference electrode. Each colour represent a unique $V_{G S}$ 47

Figure 2.21 - DNA functionalization and hybridization on a BioFET 49

Figure 3.1 - Layout design of the bioFET devices. Devices under test are shown in the middle arrays on both sides of the chip. 55

Figure 3.2 - Process flow diagram of the bioFET fabrication process..........................56 Figure 3.3 - Cross section of typical LOCOS profile fabricated at Carleton University Fabrication Laboratory; The active region is visible in the middle, and the field oxide areas surrounding it. 57

Figure 3.4 - Process flow diagram of the bioFET post-processing steps. 61

Figure 3.5 - SEM cross section of the gate and adjacent area after the wet etch of polysilicon. 64 
Figure 3.6 - Drain to Substrate diode characteristics of several bioFET sensors.

Figure $3.7-\mathrm{C}-\mathrm{V}$ characteristics of a fully processed device with a gold-chromiumpoly-oxide gate. The channel region operation can be verified as it passes through accumulation, depletion, and inversion modes of operation.

Figure 3.8 - Sub-threshold plot of both types of the fully processed devices.

Figure $3.9-\mathrm{I}-\mathrm{V}$ characteristic plots of various fully post-processed devices with and without poly layer on their gates 70

Figure 3.10 - Top view of the fabricated and post-processed bioChip. The devices under test are visible in the middle rows. All the devices to be measured are exposed to the electrolyte solution inside the glass ring. 71

Figure 4.1 - Overview of the experimental setup. 73

Figure 4.2 - Schematic of the measurement system.

Figure 4.3 - Threshold voltage shift extraction from the shift in $\sqrt{\mathrm{I}_{\mathrm{DS}}}$ vs. $\mathrm{V}_{\mathrm{GS}}$ curve for a fully processed bioFET device. 78

Figure 4.4 - Reference electrode stabilizing over time with respect to a standard electrode. Each curve on the plots shows the test at the beginning of each measurement day. 82

Figure 4.5 - Comparison of the reference electrode stabilization behaviour (with respect to a standard electrode) in two solutions with different ionic strengths measured on the same date.

Figure 4.6 - An illustration of the bioFET functionalization through thiol linkers, passivating the $-\mathrm{OH}$ groups at the sensor surface 86 
Figure 4.7 - An illustration of the bioFET hybridization at the surface of the sensor .... 87 Figure 5.1 - (a) Measurement of the output current, $\mathrm{I}_{\mathrm{DS}}$, over time before functionalization for all 4 devices. The points where the transitions were made can be seen by the wine and olive pointers on the plot for the $2 \mathrm{X}$ to $0.1 \mathrm{X}$ switch, and $0.1 \mathrm{X}$ to $2 \mathrm{X}$ switch, respectively; (b) Threshold voltage change vs. time before functionalization. The Solid lines show the calculated threshold voltage changes based on the behaviour of the devices in $2 \mathrm{X}$ solution, while the dashed lines are based on their behaviour in $0.1 \mathrm{X}$ solution. 93

Figure 5.2 - (a) Output current, I $\mathrm{DS}_{\mathrm{DS}}$ s. time after functionalization; (b) Threshold voltage $v s$. time after functionalization. 96 Figure 5.3 - (a) Output current, IDS vs. time after hybridization; (b) Threshold voltage vs. time after hybridization 98

Figure 5.4 - (a) Output current, IDS vs. time before functionalization; (b) Threshold voltage $v s$. time before functionalization. 102

Figure 5.5 - (a) Output current, I $\mathrm{DS}$ vs. time after functionalization; (b) Threshold voltage $v s$. time after functionalization 104 Figure 5.6 - (a) The output current, IDS vs. time after hybridization; (b) Threshold voltage drift $v s$. time after hybridization. 107

Figure 5.7 - The summary of the measurement process in bioFETs without poly, based on the calculated $\Delta V_{T}$ for each device 110

Figure 5.8 - The summary of the measurement process in gold coated poly gate bioFETs, based on the calculated $\Delta \mathrm{V}_{\mathrm{T}}$ for each device 113 


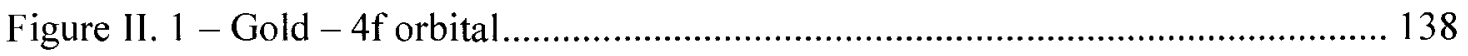

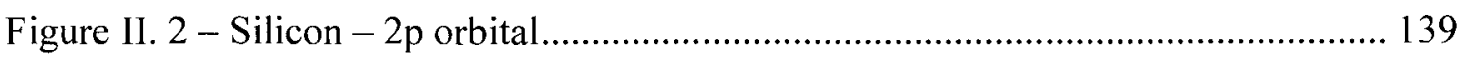

Figure II. 3 - Carbon - 1s orbital............................................................................ 139

Figure II. 4 - Oxygen - 1s orbital..................................................................... 140

Figure II. 5 - Phosphorus - 2s orbital....................................................................... 140

Figure II. 6 - Phosphorus - 2p orbital ............................................................... 141

Figure II. 7 - Nitrogen - 1s orbital _....................................................................... 141

Figure II. 8 - Sulphur - 2p orbital .................................................................... 142 


\section{List of Tables}

Table 5.1 - Calculated values of $\beta$ for each device in $0.1 \mathrm{X}$ and $2 \mathrm{X}$ buffered solutions on bare gold surfaces

Table 5.2 - Summary of threshold voltage shifts before functionalization. 94

Table 5.3 - Calculated values of $\beta$ for each device in $0.1 \mathrm{X}$ and $2 \mathrm{X}$ buffered solutions after functionalization.

Table 5.4 - Summary of threshold voltage shifts after functionalization. 97

Table 5.5 - Calculated values of $\beta$ for each device in $0.1 \mathrm{X}$ and $2 \mathrm{X}$ buffered solutions after hybridization. 97

Table 5.6 - Summary of threshold voltage shifts after hybridization 99

Table 5.7 - Calculated values of $\beta$ for each device in $0.1 \mathrm{X}$ and $2 \mathrm{X}$ buffered solutions before functionalization. 100

Table 5.8 - Summary of threshold voltage shifts before functionalization. 101

Table 5.9 - Calculated values of $\beta$ for each device in $0.1 \mathrm{X}$ and $2 \mathrm{X}$ buffered solutions after functionalization. 103

Table 5.10 - Summary of threshold voltage shifts after functionalization 105

Table 5.11 - Calculated values of $\beta$ for each device in $0.1 \mathrm{X}$ and $2 \mathrm{X}$ buffered solutions after hybridization. 106

Table 5.12 - Summary of threshold voltage shifts after hybridization. 108 


\section{Glossary}

ssDNA

dsDNA

DNA Denaturation

DNA Functionalization

DNA Hybridization

Miss-match

Thiol group

Linker group

Nernstian behaviour
Single-stranded DNA sequence

Double-stranded DNA sequence

Breaking the hydrogen bonds of a dsDNA molecule, resulting in separation of the two ssDNA sequence

Immobilizing a ssDNA sequence on a surface

Bonding two ssDNA sequences together through their hydrogen bonds, i.e. the reverse denaturation process

DNA hybridization involving two non-complementary ssDNA sequences

An organic molecule consisting of an alcohol whose oxygen atom has been changed with a sulphur

A molecule (normally not charged) which allows attachment of DNA sequences into a surface

The equilibrium state of an electrode when it follows the Nerst equation, while the concentration of the ions involved the reaction change. 


\section{Chapter 1}

\section{Introduction}

\subsection{Motivation}

Most recently, there has been a peak of interest in the field of biomedical sensors to detect biological species, particularly pathogens, such as bacteria and viruses in order to facilitate the detection of diseases. Such methods make use of detecting the samples' biological molecules such as proteins and Deoxyribonucleic acid (DNA) strands. Popular existing methods of detection and analysis, such as optical laser DNA microarrays and chemical testing using various reagents require labelling with labels such as fluorescent dyes. Although these methods prove to produce reliable and accurate results, they are rather expensive, often requiring experienced technicians to operate complex machinery. Therefore, biosensing methods relying on biological or chemical interaction with an electrical interface have been found to be attractive substitutes, owing to their simplicity, disposability, and low cost. They also remove the requirement for labelling.

There exist a large number of label-free biosensors for specific applications. A promising type of biosensors based on electronic detection of charged biological molecules, such as DNA strands, is the use of metal oxide semiconductor field-effect transistors (MOSFETs). MOSFETs are used in countless applications in electronics, using conventional complementary metal oxide (CMOS) compatible fabrication 
processes. MOSFETs utilize a metal gate on an insulator layer, normally silicon dioxide, to modulate the surface charge of the underlying semiconductor, typically silicon. Changes in the charges at the surface of the device result in changes in the conductivity through electric fields penetrating into the semiconductor. Through coupling the gate area to an electrolyte solution, the FET structure can be transformed into an ion-selective FET (ISFET), which can detect changes of the ions or charged molecules in the electrolyte. ISFETs have been under study for several decades, and can be fabricated simply in a microarray.

An ISFET whose surface has been populated with charged biological molecules, such as proteins or DNA strands, is a sensor and called a bioFET. Sensing is possible through immobilizing DNA, ribonucleic acid (RNA), or peptide nucleic acid (PNA) molecules, and hybridizing with complementary DNA molecules. The bioFET sensors are compatible with conventional processes of microelectronics fabrication and postprocessing. So far, most of the research has concentrated on sensing on the oxide or gold interface of the $<100>$ silicon crystal based bioFET. Such sensors are practical and show reasonable sensitivity; however, there has been research on alternative sensors to achieve larger hybridization signals.

A promising alternative to the conventional $<100>$ silicon crystal based sensors is direct immobilization of probe molecules on the semiconductor, particularly, $<111>$ silicon crystal. Owing to its atomic structure, $<111>$ orientation allows creation of a larger number of binding sites available at the surface of the semiconductor for DNA immobilization. Through direct immobilization of probe molecules on the semiconductor, 
$<111>$ silicon based bioFETs are expected to provide superior sensitivity to changes in the surface charges. Superiority of the $<111>$ orientation is also expected when used in a silicon on insulator (SOI) structure. However, if the silicon dioxide insulator layer is left in place in either orientation, a metal surface such as gold also offers a density of binding sites.

Due to lack of self aligned properties, FETs fabricated with metal gates result in lower performance. To eliminate this issue is the metal deposition as a post-processing step. One of the conventional methods is using polysilicon (a self aligned material) during the fabrication process and removing this layer right before metal deposition process. However, the poly removal step, usually a wet etch process, normally causes minor damage to the gate structure which can reduce the sensitivity of the bioFET sensor. Therefore, if poly layer is kept in place and the exposed metal layer is deposited on top of it, damage due to the wet etch process is eliminated, and the device performance, i.e. sensor sensitivity, is not compromised.

This research focuses on the fabrication and testing of silicon $<111>$ orientation based bioFETs with gold surfaces deposited on the polysilicon gates, and comparing them to bioFETs with the removed polysilicon layer.

\subsection{Thesis Contribution}

In the past few decades various methods have been employed to detect biological samples. Different in sensing mechanism, all these methods have common objectives: faster, more sensitive, and more reliable low cost sensors, which can detect targets in real 
time. Although portability has not been a concern so far, future needs will require transportable sensors as well. A more thorough discussion of the various methods of DNA detection is elaborated in chapter 2. The biosensor presented in this work employs detection methodology using field-effect transistors, which can be integrated with signal processing circuitry to produce a low cost portable sensor.

The research on bioFETs at Carleton University has been in coordination with the National Research Council of Canada. The research has been based on the bioFET detection methodology modeled at National Research Council of Canada. Dr. Dolf Landheer, and Dr. Ross McKinnon completed much of the development of the CarletonNRC bioFETs [1-4]. Earlier research was performed by Alasdair Rankin [5]. The work presented in this thesis employs the previous work using the bioFETs fabricated and post-processed at Carleton University and the National Research Council of Canada based on the layout design of Ryan Griffin. Additionally, this work illustrates testing DNA functionalization and hybridization processes on gold surfaces of silicon $<111>$ based bioFETs where the polysilicon layers of the gates were not removed, and compares them to the bioFETs with no polysilicon layer. The team lead by Dr. Maria DeRosa of the Department of Chemistry at Carleton University kindly provided the functionalization and hybridization methodology based on the thiol-gold binding at the sensor surface.

\subsection{Outline}

Chapter 2 provides background information about DNA structure, DNA binding, and current methods of DNA sensing. Beginning with an elaboration on the basic 
semiconductor physics, a MIS structure is outlined; based on which MOSFET and ISFET fundamentals are outlined. Ultimately, the DNA bioFET is introduced. A brief review of surface chemistry and a review of reference electrodes are also included.

Chapter 3 describes the fabrication and post-processing procedures, at Carleton University and National Research Council of Canada, respectively. This chapter illustrates how the bioFET devices are produced using conventional Complementary MOS (CMOS) process, and converted into ISFETs well-suited to detect biological species. Preliminary device testing and packaging is also described.

Chapter 4 illustrates how the bioFET measurement setup has been prepared and how the experimental procedure is carried out. Difficulties resulting in reduction of the device sensitivity and increase in the noise are discussed, and it is explained that how they have been solved.

Chapter 5 provides a summary of the measurements and the results, along with a discussion on the results. It also includes a comparison between the two type of bioFETs fabricated, and measured.

Chapter 6 presents the conclusion of this thesis, as well as suggestions for the future research in bioFET based sensors. 


\section{Chapter 2}

\section{Background}

\subsection{DNA}

Deoxyribonucleic acid, DNA, is the key molecule that stores the information for the biological functions of all known living organisms. DNA determines the information to produce the structural and regulatory proteins necessary for tissue and cellular architecture and performance [6]. What we know today of the DNA molecule is based on the preliminary work of James D. Watson, and Francis Crick. Such a structure is a straight, slender, right handed double helix which is composed of two strands held together through a complementary base pairing, called Watson-Crick base pairing [7].

The unique genetic information accumulated in this structure is stored in a linear polymer formation composed of subunits called nucleotides. Each nucleotide consists of a backbone made from sugar and phosphate groups, along with a distinct base, exclusively patterned. This pattern allows creation of diverse series of information stored in a DNA sequence. The number of bases in a DNA molecule is typically in the range of millions. The ability to read this information sequence is helpful to scientists in various fields such as molecular biology, medicine, biochemistry, agriculture, etc. 


\subsubsection{Bases of Nucleic Acids}

DNA bases are categorized as purines and pyrimidines, based on the number of their heterocyclic rings.

\subsubsection{Purines}

Purines are heterocyclic aromatic bases with a purine ring, shown in Figure 2.1, composed of two 6 member and 5 member nitrogen-containing carbonic rings attached at position 4 and 5 of the 5 member ring. The two most common purines are Adenine and Guanine, which exist in any nucleic acids (DNA, RNA, and PNA). Other purines include caffeine, hypoxanthine, isoguanine, theobromine, uric acid, and xanthine.

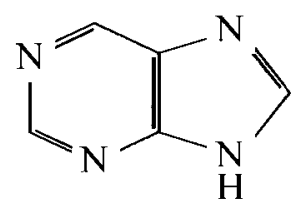

Figure 2.1 - Purine ring

As seen in Figure 2.2 adenine has an amino group $\left(-\mathrm{NH}_{2}\right)$ on $\mathrm{C} 6$ (carbon atom at position 6) of the purine ring, whereas guanine has an amino group at the C2 position, and a carbonyl group $(=\mathrm{O})$ at the $\mathrm{C} 6$ position of the purine ring. 
<smiles>NC1=NCNC2NCNC12</smiles>

(a)<smiles>NC1NC(=O)C2NCNC2N1</smiles>

(b)

Figure 2.2 - Purine bases: (a) Adenine; and (b) Guanine.

\subsubsection{Pyrimidines}

Pyrimidines, shown in Figure 2.3 and similar to purines, are heterocyclic aromatic bases which have two nitrogen atoms at position 1 and 3 of the carbonic ring. Cytosine, Thymine, and Uracil are the three nucleobases pyrimidine derivatives found in nucleic acids. While cytosine is present in many nucleic acids, DNA sequences usually do not contain any uracil, but have thymine instead.

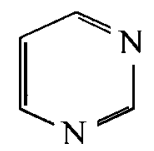

Figure 2.3 - Pyrimidine ring

Cytosine is a pyrimidine derivative with a carbonyl group at $\mathrm{C} 2$, and an amino group at $\mathrm{C} 4$ position. Thymine is also another nucleobase found in DNA sequences, with two carbonyl groups at $\mathrm{C} 2$ and $\mathrm{C} 4$ position, as well as a methyl group at $\mathrm{C} 5$ position. Uracil has a similar structure to thymine, except it does not contain the methyl group. As

mentioned earlier, uracil is not usually found in DNA, and is instead a component of RNA where it usually replaces thymine. The three types of pyrimidines are presented in Figure 2.4. 
<smiles>Nc1cc[nH]c(=O)n1</smiles>

(a)<smiles>Cc1c[nH]c(=O)[nH]c1=O</smiles>

(b)<smiles></smiles>

(c)

Figure 2.4 - Pyrimidines bases: (a) Cytosine; (b) Thymine; and (c) Uracil.

The location of the hydrogen bond donor and acceptor groups shapes the purines and pyrimidines molecules in such an exclusive way that each molecule can store distinctive information in the cell. Since both the purines and pyrimidines molecules are composed of flat rings, they can stacked on top of each other, providing a firm base.

\subsubsection{Sugars Present at Nucleic Acids}

Ribose and Deoxyribose, shown in Figure 2.5, are the source of sugar in biochemical synthesis of purines and pyrimidines. They are both blended based on the $\beta-$ D-ribose rings. A $\beta$-D-ribose ring is an aldopentose (5-carbon sugar) with a hydroxyl group $(-\mathrm{OH})$ on each carbon. Deoxyribose has the same structure as ribose, except the hydroxyl group at the $\mathrm{C} 2$ position is replaced by a hydrogen atom $(-\mathrm{H})$. While ribose is found in RNA sequences, the DNA sequences are composed of deoxyribose. 


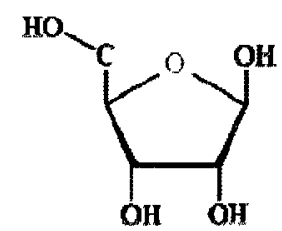

(a)

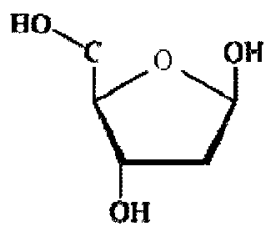

(b)

Figure 2.5 - (a) $\beta$-D-ribose; and (b) $\beta$-D-2-deoxyribose, sugars present at nucleic acids.

\subsubsection{Nucleosides}

The combination of the base pairs with sugars creates nucleosides, adenosine, guanosine, cytidine, and thymidine. In DNA sequences, the pairing of the base pairs with a $\beta$-D-2-deoxyribose sugar produce deoxyadenosine, deoxyguanosine, deoxycytidine, and deoxyadenosine respectively, as presented in Figure 2.6.

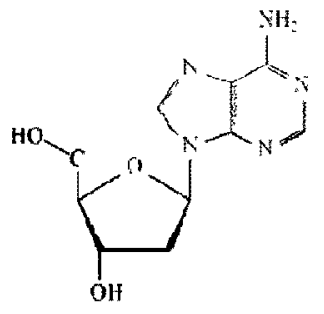

(a)

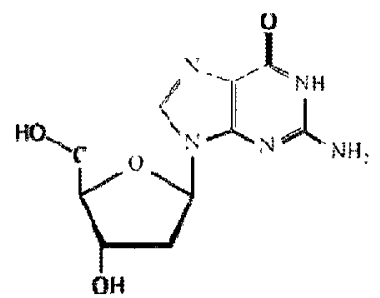

(b)

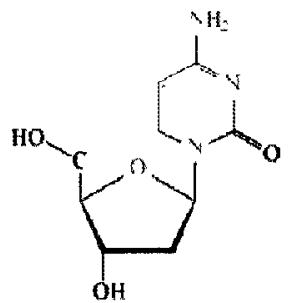

(c)<smiles>CC(CN(CO)C1CC(O)C2OCC1O2)C(N)=O</smiles>

(d)

Figure 2.6 - Nucleosides: Deoxyadenosine; (b) Deoxyguanosine; (c) Deoxycytidine; and (d) Deoxythymidine. 


\subsubsection{Nucleotides}

Nucleotides, shown in Figure 2.7, are fundamentally products of coupling nucleosides with a phosphate group and are found in RNA and DNA sequences. The nucleotide can have one, two, or three phosphate groups designated $\alpha, \beta, \gamma$. DNA sequences mostly consist of monophosphate ribonucleosides, whereas in RNA triphosphate (converted from monophosphate to diphosphate and subsequently triphosphate) groups exist.

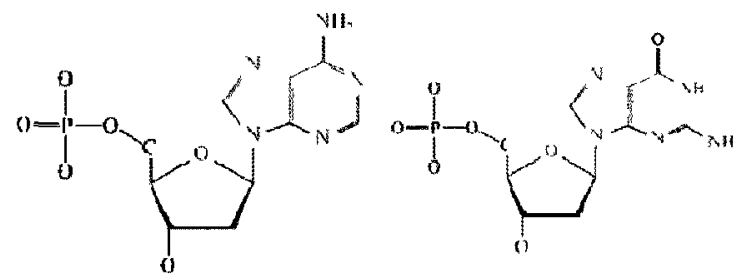

(a)

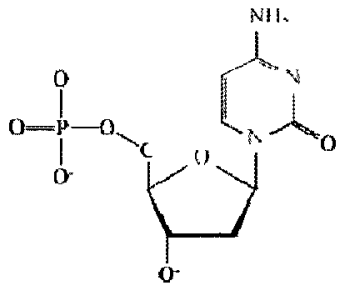

(c)

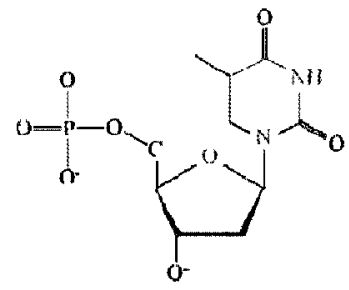

(d)

Figure 2.7 - Nucleotides: Phosphate deoxyadenosine; (b) Phosphate deoxyguanosine;

(c) Phosphate deoxycytidine; and (d) Phosphate deoxythymidine.

\subsubsection{DNA Sequences}

\subsubsection{Single-Stranded DNA}

The nucleotides can be polymerized together through the phosphate bonds to form a nucleic acid. Such bonding is called a phosphodiester bond and is formed by coupling of a phosphate at $\mathrm{C} 5$ of a nucleotide to the hydroxyl group of $\mathrm{C} 3$ at the neighbouring nucleotide to form an ester bond (-CO-O-), eliminating a water molecule. The formed 
structure, which exists in all DNA (and RNA) sequences is a polynucleotide that has a position C5 free phosphate (tri-phosphate in RNA) group on one end, called the 5 ' end, and a position $\mathrm{C} 3$ free hydroxyl group $(-\mathrm{OH})$ on the other end, called the 3 ' end. A polynucleotide sequence creates a Single Strand DNA (ssDNA). A DNA sequence with $n$ number of nucleotides is called an n-mer DNA sequence. A DNA sequence is conventionally written in the 5' to $3^{\prime}$ direction.

As it can be seen from the Figure 2.8 , the polynucleotide molecule has a unique structure, which makes it polar. This is due to the presence of the phosphate groups on the backbone of the polymer, which on the whole makes the backbone have negative polarity in an ambient $\mathrm{pH}$ value of approximately 7 . The acid ionization constant, $\mathrm{pK}_{\mathrm{a}}$, of the phosphate group is very close to one which makes it negatively charged in aqueous solution. The opposite side of the phosphate groups are hydrogen rich groups that are prepared to donate or accept electrons and bond in an aqueous environment.

\subsubsection{Watson-Crick Base Pairing}

Adenine can only be coupled with thymine, whereas guanine can only be couple with cytosine. Such attachments are called base pairings. Looking at the chemical structure of the purines and pyrimidines it can be seen that all bases have both nitrogen containing groups, or keto oxygen (-CO-). If a purine and a pyrimidine are brought close enough together in an aqueous solution, nitrogen containing groups in both base bases can act like donors, whereas heterocyclic nitrogen or the keto oxygen can act like acceptors. The result is a hydrogen bonding between the purine and pyrimidine bases, which is stronger than van der Waals forces, but weaker than ionic or covalent bonds. 


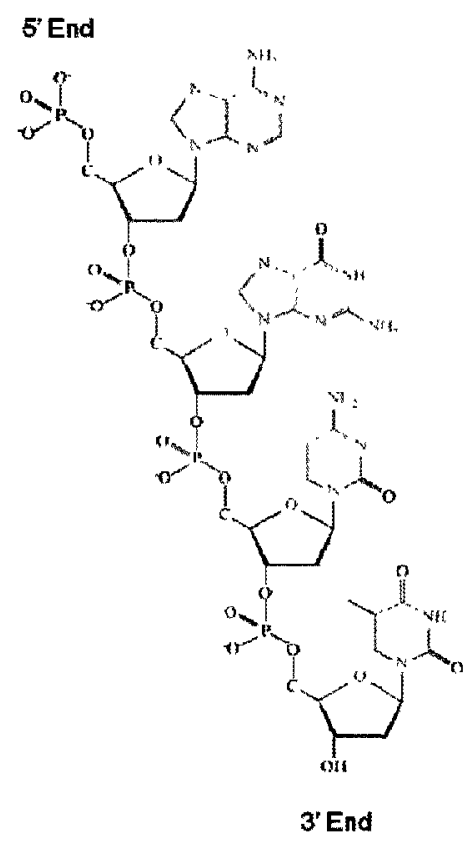

Figure 2.8 - A Simple polynucleotide present in DNA.

Adenine and thymine each have only two "free" hydrogen atoms ready to be bonded to the neighbouring nucleoside, whereas guanine and cytosine have three. Hence, there is a strict match between the number of bonds available, and due to this match, the adenine - thymine base pair (AT) and guanine - cytosine base pair (GC) are matched (as shown in Figure 2.9), whereas AC or GT are not. Therefore, AT and GC pairs are more stable than AC and GT pairs [8-9].

Since the GC base pair has more hydrogen bonds than the AT pair, the GC pair is stronger and more stable. As a result, a DNA sequence with more GC pairs than AT pairs (high GC) is inclined to be more stable, and the hydrogen bonds will break slower. Despite the difference in the hydrogen bonds, AT and GC pairs have similar dimensions. 


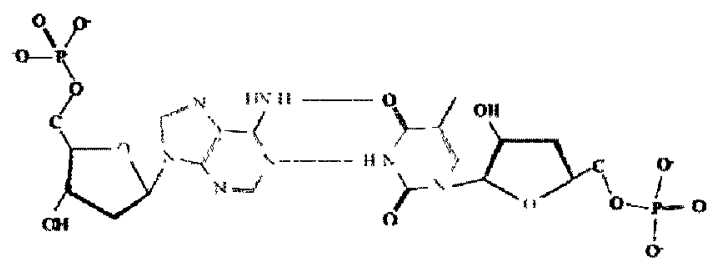

(a)

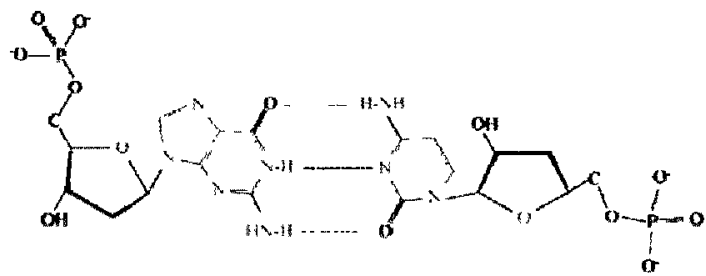

(b)

Figure 2.9 - Watson-Crick base pairs: (a) Adenine - Thymine pair; and (b) Guanine - Cytosine pair.

\subsubsection{Double-Stranded DNA}

If a sequence of nucleotides stacked on each other, such as a ssDNA sequence, is approached to another sequence with matching bases aligned in an anti parallel trend (assuming conditions are all met), the hydrogen bonds between the matching bases lead the sequences to bond to and wind around each other [10]. The bonding creates a right handed cylindrical helix structure, which is a Double-Stranded DNA sequence, which was discovered by Watson and Crick in 1952 [7], [11-12]. An example of this bonding is shown in Figure 2.10.

As mentioned earlier, both the bases and the base-pairs are planar, and thus stacking the pairs on top of each other contributes significantly to the overall stability of the double helix. The pitch of the helix is $3.4 \mathrm{~nm}$, and there are about 10 pairs per turn, which gives an average $36^{\circ}$ angles between consecutive base pairs. Since the strands have one to one matching bases; i.e. they completely match each other by pairing, they are 
complementary strands. For any distinctive single strand there is one and only one complementary strand running in the opposite direction. Moreover, a miss-pairing between two non-complementary ssDNAs is possible. However, this pairing is not stable, and the hydrogen bonds will eventually break.

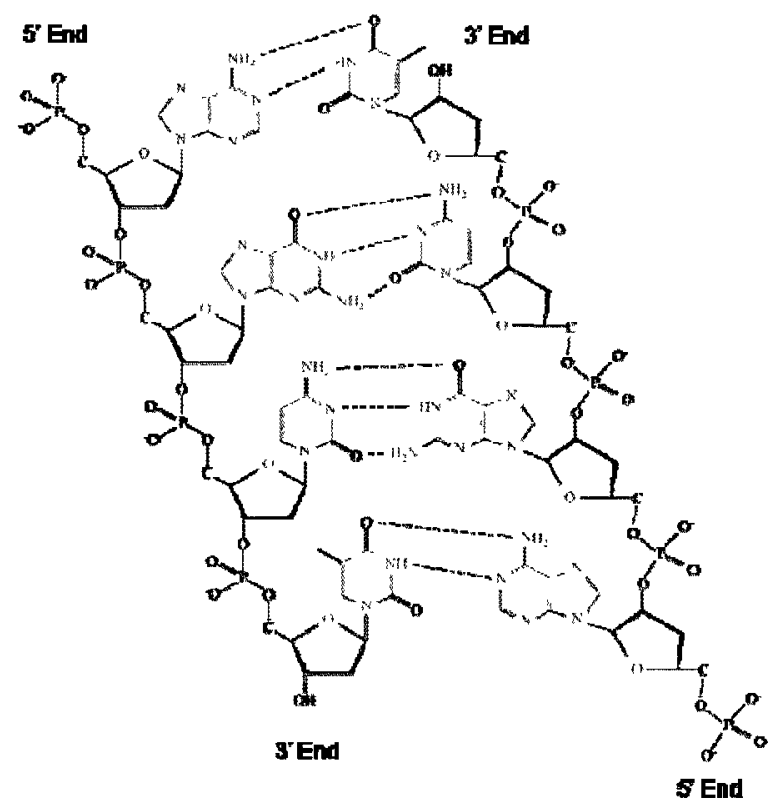

Figure 2.10 - A 4-mer Double-Stranded DNA sequence (ACGT).

In the double helix formation the nucleotides are clustered close to the centre of the structure. This creates a strong backbone holding the single strands together by noncovalent hydrogen bonds. As mentioned above, the richer the sequence is in GC base pairs, the stronger the backbone (hence, the formation) will be. The backbone is enclosed by the phosphate groups surrounding the whole formation. Not only do they help the sequence stay stable, but they also form a cloud of negative electric charge around the 
whole structure, when ionized in an aqueous solution. This effect is illustrated in Figures 2.10 and 2.11 .

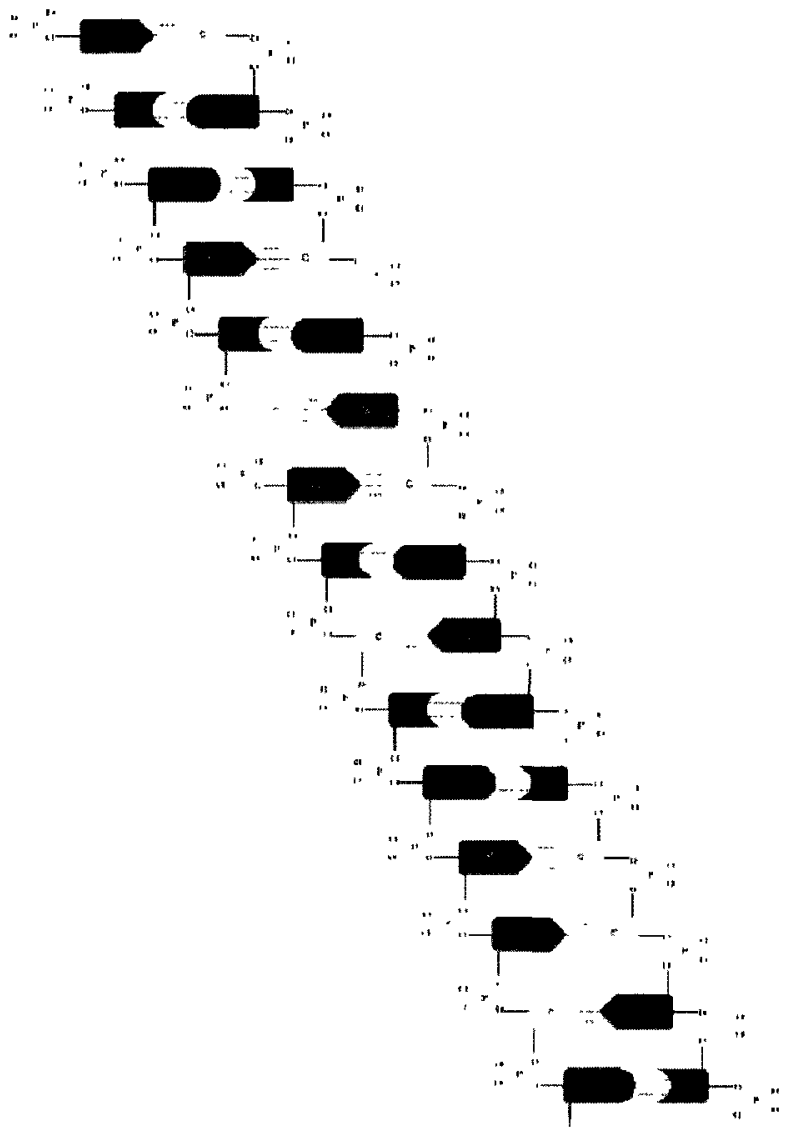

Figure 2.11 - A 15-mer Double-Stranded DNA sequence (GTAGTCGTCTAGGCA).

\subsubsection{Stability of a Double Stranded DNA Sequence}

A dsDNA molecule is held together at its backbone through the non-covalent hydrogen bonds. The hydrogen bonds are stronger than van der Waals forces, but weaker than ionic or covalent bonds. Since the hydrogen bonds are not very strong, they are easily broken. Breaking the hydrogen bonds in one base can result in separation of 
nucleosides in that sole pair; conversely, it might influence the hydrogen bonds of the neighbouring pairs to loosen, resulting in a local denaturation.

The two single stranded molecules formed from the denaturation process are typically stable, even if the denaturing conditions are eliminated. The single strands commonly do not renature back into the original double-stranded molecule, instead they form tangled balls. However, by adjusting the denaturing conditions, the two complementary strands can again be forced to hybridize or renature and form a double helix. The renaturation property is used as the foundation of the DNA microarray technology [13]. The following conditions influence the stability of a dsDNA sequence:

\section{a) Temperature Effects}

The ambient temperature influences the hydrogen bonds and increasing the temperature causes the hydrogen bonds to gradually loosen and ultimately break. The detachment of the dsDNA into two ssDNA strands due to the ambient heat is called melting, and is described by the value of $T_{m}\left({ }^{\circ} \mathrm{C}\right)[14]$. In nature at $T_{m}$, half of the population of the nucleic acid, called oligonucleotide, is denatured into the single stranded form and the other half is still in the double stranded shape. However, by convention, it is considered that the denaturation starts at $T_{m}[15]$.

The $T_{m}$ value of a sequence varies based on the sequence and the ambient electrolyte's salt concentration in the solution. The higher the sequence concentration $\left(C_{t}\right)$ is, the higher duplex formation will be and therefore the higher $T_{m}$ will be. Salt concentration in the solution affects the ionic strength of the solution. As it will be 
explained shortly, the salt stabilizes the double strand formation. Furthermore, the more $\mathrm{GC}$ pairs that are present in the sequence, the stronger the base pairs, are bonded together, and thus the higher the $T_{m}$ value is. However, at extreme temperatures above $T_{m}$, the covalent bonds stacking the bases also break [16].

\section{b) Hydrogen Bonds}

While the primary structure of the DNA is determined by the covalent bonds between the neighbouring nucleosides, the hydrogen bonds in the DNA backbone outline the three-dimensional shape of the sequence. A higher number of bonds present in the DNA structure, and also stronger bonds, result in more stability of the entire DNA formation.

\section{c) pH Effects}

At extreme $\mathrm{pH}$ values, above $\mathrm{pH} 10$ or below $\mathrm{pH} 3$, the hydrogen bonds as well as the ionic bonds between the bases, phosphate groups, and ribose sugars tend to break. Not only is the dsDNA sequence denatured at such $\mathrm{pH}$ values, but the nucleoside sequences also deform and nucleosides begin to divide.

\section{d) Ionic Strength Effects}

The reassociation rate increases as the ionic strength (particularly concentration of sodium and potassium ions) in a hybridization reaction is increased. The ions shield the negative charges of the phosphate backbone, and screen the electrostatic repulsion between the two backbones [17-18]. 


\section{e) Hydrophobic Effects}

Purine and pyrimidine bases are hydrophobic molecules and their polarity is generally influenced in aqueous environment. Since they are enclosed inside the cylindrical helix by the ribose sugars and phosphate groups, they are well protected from the hydrophobic effects on them, increasing the stability of the sequence.

\subsubsection{DNA Sequence Binding}

\subsubsection{DNA Denaturation}

The effects mentioned above control the stability of the dsDNA sequence. If the DNA molecule instability exceeds a level, the sequence will denature. For example if the temperature increases above $\mathrm{T}_{\mathrm{m}}$, the hydrogen bonds weaken and the two strands eventually separate, resulting in a total denaturation [15].

Other factors also affect the $T_{m}$. For instance, if in the solution where the DNA is dissolved the ionic strength is relatively high, the salt guards the electrostatic interferences and provides higher stability to the helix. Conversely, if the ionic strength is reduced, the negative charges on the phosphate groups repel each other. This lowers the helix's stability. Hence, a lower energy level (i.e. $T_{m}$ ) is required to separate the strands and melt the DNA molecule.

In the same fashion, increasing $\mathrm{pH}$ to an extremely high level (above $\mathrm{pH} 12$ ), or reducing it to increasing $\mathrm{pH}$ to an extremely low level (below $\mathrm{pH}$ 2) effects the cloud of negative charges surrounding the helix, as well as the covalent bonds stacking the bases. Such weak bonds cannot shield the DNA backbone and the hydrogen bonds also weaken 
by being ionized. This leads to destabilizing the base pairs, their separation, and ultimately helix denaturation.

\subsubsection{DNA Immobilization}

The technique of attaching a dsDNA onto a solid substrate using surface chemical reactions is called immobilization. Among metal surfaces, a gold substrate is the most favourable one, using a thiol group as an interface for immobilizing a sequence. In printing microarrays schemes such as photolithography, ink-jet printing, electro-printing, and laser writing are commonly used. In addition to chemical immobilization, physical immobilization, such as physical adsorption, entrapment in polymeric gels, and containment within semi-permeable membranes are widely used for immobilization of optical biosensors [19].

In DNA immobilization, a self-assembled monolayer (SAM) can operate as an interface between the sensor and the sample's receptor molecules as it covalently bound to both of them [21]. The SAM can be made such that one of its ends reacts covalently with the biosensor surface, and the other end has a reactive group which can bond to the sample sequence. Using different SAMs permits simultaneous detection measurements on different sequences. Moreover, the depth of a SAM interface controls the distance between the biosensor surface and the sample sequence [22].

\subsubsection{DNA Hybridization}

Forming any dsDNA molecule by attaching two ssDNA sequences together is called DNA hybridization. In the other words, DNA hybridization is the process of 
reversing denaturation procedure of any dsDNA molecules. If the single-strand sequences are complementary, after bonding together, they will form a double helical structured dsDNA sequence [23-28].

For more information about principles of DNA structure and the hybridization process, the reader is referred to [6] and [29].

\subsubsection{Microarray Technology}

Recently, researchers have been able to immobilize (or spot) thousands of different ssDNA molecules, called probe molecules, in a grid shaped pattern on a flat surface, constructing a DNA microarray. Each grid location, a spot, is composed of various identical replicas of a particular specific DNA sequence. The objective of the microarray technology is to identify and calculate the concentration of unknown sequences in a sample analyte solution [30-37].

The microarray is introduced to a sample of different ssDNA sequences, targets, and incubated for a period of time. Through the incubation, target molecules floating in the solution, are exposed to the probes and are capable of binding onto a complementary probe on the spot. If a target binds to a complementary probe, it will hybridize with it, and will form a dsDNA sequence. If a target and probe pair share a matching sequence, but do not have the same length, they will still bond [38].

A higher number of complementary probes present at the spots results in a higher number of target molecules that will hybridize to them [39]. However, during incubation a small fraction of the targets loosely bind to probes with different sequences, non- 
complementary probes. Such bindings are non-specific bindings. They are the result of cross-hybridization and are fairly unstable. After incubation, the microarray is washed with a detergent buffer, and all the unbound target molecules are swept away. The buffer also causes most of the non-specific bindings to denature and the targets are carried away as well. However, a very small number of cross-hybridized target-probe bonds (with the highest ratio of matching pairs to non-matching) remain. But they will unbond over a short period of time if they are left in a buffered solution [40-41].

In order to determine the concentration and type of each specific target sequence in the sample, the targets could be attached to labels, predominantly fluorescent dyes [4243] or radio isotopes [44], before the incubation. Upon washing, the microarray is scanned for the fluorescent [45-47] or radioactive [44] signals. Since the probe sequence on each spot in the array is known, the presence of labels determines the concentration, as well as the type of the target sequence. The sensitivity of a microarray or the smallest accurately detectable target concentration is called the limit of detection [48-50]. Incorporating the benefits of photolithography techniques, the spotting can be very dense; hence, a higher number of target/probes can be investigated in a single measurement, resulting in a better limit of detection [51-55]. Furthermore, the ability of a microarray to distinguish among various DNA target sequences is called specificity [56-57]. A very small number of non-specific bindings results in a higher microarray specificity. Assuming all other conditions (initial concentration of targets, incubation temperature, washing steps, etc.) flawlessly met, close length matching of probes and targets immensely improves the microarray's limit of detection and specificity [58]. 


\subsection{Biosensors}

As defined by the International Union of Pure Applied Chemistry (IUPAC) in 1992, "a biosensor is a device that uses specific biochemical reactions mediated by isolated enzymes, immunosystems, tissues, organelles, or whole cells to detect chemical compounds usually by electrical, thermal or optical signals" [59]. Biosensors are fundamentally analytical devices translating the biological information into a quantitatively measurable signal [60]. The generated signal can be in the forms of acoustic, optical, or electrical responses [61-62]. The process of responding to the biological information is carried out by chemical or biological reactions through bioreceptors, sensitive materials constituted in biosensors to recognize the reaction event [63]. A biosensor system or device consists of two discrete parts: a biological recognition component such as antibodies, enzymes, proteins, oligonucleotides and cells, and a signal transduction building block connected to a data acquisition and processing system.

Studies on the utilization of biosensors for DNA detection are relatively recent but the research is increasing swiftly. Particularly during the last decade, there has been a significant amount of interest and development in the area of DNA molecular engineering, i.e. DNA molecular synthesis technology, owing to its practical applications in many regions of the nanotechnology and biotechnology fields. Biosensors are employed in a wide range of areas including environmental monitoring [64-65], molecular exchange screening for drug discovery [66-67], clinical diagnosis [68], molecular biology [69-70], and genetics [71-72]. New biosensor technologies are holding a promising future for low cost, one step biological information detection. Stability, 
sensitivity, specificity, and ability of real-time measurements are the main factors of superiority for future commercial biosensors [73].

In general, biosensing techniques can be mostly categorized into label-based and label-free techniques. Each of these methods is sub-classified based on its underlying physics. Selectivity and label tuning abilities allow label-based techniques to demonstrate more sensitivity than the label-free methods. However, they generally involve a costly, labour intensive and time consuming labelling step. There is also a large possibility that during the sample labelling, its binding properties are severely modified, which makes such labels unreliable. In contrast, the label-free techniques do not require any labelling; therefore, detection can be performed in a real time approach, which is comparatively quicker. Absence of labels also reduces the chances of contamination or cross-hybridization. Additionally, the stability of the label-free techniques is on average higher than the label-based detection methods [74-75].

\subsubsection{Label-based Sensing Methods}

Label-based methods use labels or tags in order to sense a particular analyte present in an ambient background of other materials. Because of the tags' selective property, measurements in high noise background are achievable. The long-established practice of biological sensing area is based on employing optical (primarily radioactivity and fluorescence) measurements. However, chemiluminescence is widely used as well. The most commonly used techniques are: 


\section{a) Radioactivity}

Probe molecules are attached onto a firm base surface by a covalent bond, either directly or using a cross-linker. Then probe molecules are targeted by the targets in an aqueous solution (or in gaseous state) which themselves are attached to radioactive isotope labels. The targets (such as antigens) bond to the probe molecules (such as antibodies) and hybridize. The hybridization is detectable using radioactive scanning of the labels [44].

Although using the radioactive isotopes (radioimmunoassay, RIA) is extremely sensitive, due to the safety concern it is not widely publicly accepted. A need for a long exposure time reduces the number of samples which could be read. On the other hand, since the bonds are not reversible, the sample cannot be reused. This makes the samples practical only for a single measurement, which increases the cost.

\section{b) Fluorescence}

Fluorescence based detection is very comparable to the radioactive method, apart from the use of fluorophores dyes instead of radioactive isotopes [76]. Because of its stability and sensitivity, and also the availability of a large variety of fluorescent dyes, this technique is by far the most widely common practice comparing to other label-based detection methods [34]. Furthermore, with the development of microarray techniques in the DNA analysis, fluorescence has gained more popularity [77]. On the other hand, most fluorophores react rapidly when exposed to light and ambient conditions, such as the $\mathrm{pH}$ 
value of the solution alters their sensitivity [42]. Moreover, the labels might modify the interaction affinities of the macromolecules to which they are attached [43].

\section{c) Chemiluminescence}

Enzyme-linked immunosorbent assay (ELISA), is a common technique in antigen detection. Unlabeled antigen is first captured by the immobilized monoclonal primary antibody through specific binding. A secondary detection antibody, linked to an enzyme, is then added to bind to the antigen, followed by the addition of a substrate which is converted by the enzyme into a coloured product. The rate of colour formation is proportional to the amount of the antigen present in the sample [78-79]. If antigens are attached directly onto the sensing surface, they can detect antibodies.

Since in the ELISA detection method a secondary pair of antibody and enzymesubstrate is employed, the detected signal can be boosted by the pair. Amplified detection results in a considerably higher sensitivity. The very high sensitivity of the ELISA technique is well employed in microarray-based biosensors [69].

\subsubsection{Label-Free Detection Techniques}

A label-free biosensor is essentially a biosensor which does not require use of any label molecules such as radioactive or fluorescent dyes to expose the characteristics of the target molecule. For this reason, in the label-free biosensing techniques the time, labour, and money consuming steps of labelling can be avoided. This results in more time and cost efficient measurements, which can lessen the experimental discrepancies due to operator error [80]. 


\section{a) Surface Plasmon Resonance}

Although Surface Plasmon Resonance (SPR) optical sensing technique was introduced in 1982 [81-82], it was not commercially available until the 1990's [83]. Until then the label-based detection methods were most practical and popular (and they still are) [50], [84]. Such methods required the use of antibody vs. antigen bonds for reading biological information. In the SPR technique, a laser beam is pointed toward the sample, and the critical angle of the reflected light changes as the sample presence changes the polarization of the incoming light. Based on the conditions of the surface, the intensity of light during reflection is diminished [84-86]. The hybridization of target molecules onto their complementary probes modifies the surface's refractive index properties and the angle of light absorption shows a different change than before hybridization [87-90]. Since in SPR the composition of the target is irrelevant, this technique enabled researchers to investigate a wide range of biological compositions [91-92]. On the other hand, if dealing with low molecular weight analytes, less than a thousand Daltons, the sensitivity of the detection is reduced [93].

\section{b) Mass Spectrometry}

Mass Spectrometry (MS) is an excellent technique to accurately recognize and measure the elemental composition of very complex nucleic acids. If MS is used along with electrophoresis, it can precisely identify analytes in any given sample [94]. However, since the apparatus utilized for mass spectrometry is very large, this technique is not practical for commercial and portable use. Nonetheless, the use of MS in research laboratories is very popular. 


\section{c) Acoustic Wave Sensors}

Acoustic wave sensing takes advantage of the transduction mechanism based on the visco-elasticity of the surface. Presence of the sample at the sensor surface influences the velocity of the acoustic waves traveling at and near the surface of the sensor, and cause additional attenuation in the acoustic wave. Since the thickness shear mode devices are extensively commercially available, most acoustic wave sensors have been designed based on such devices. Detection based on the acoustic wave is significantly reduced in liquid environment. Any deformation in the surface of the sensor also diminishes the detection sensitivity [95].

\section{d) Surface Stress Sensors}

Similar to the acoustic wave detectors, there have been microcantilever sensors made using measurements of surface stress to detect biological reactions [96-99]. Surface stress sensors are suitable for detecting base pair mismatches in the DNA double helix [100]. Detection could be done by various methods using piezoresistive transducers [101104], optical deflection readings [96], or capacitive detections [105]. Due their small size and ease-of-use, surface stress biodetectors can be used to make microarray structures [32]. However, these sensors are yet to be commercially available, and more research needs to be done on them [106-114].

\section{e) Electrochemical Techniques}

Electrochemical techniques make use of monitoring the reduction or oxidation reactions of the analyte of interest attached to one or more working or counter electrodes 
[115-116]. There are different methods of electrochemical measurements based on the electrodes used. Using potential, resistance, current or capacitance readings, electrochemical measurements can be done using potentiometric [60], [117-118], conductometric [119], amperometric [60], [120], or faradaic [121-125] techniques. The change in the potential between a reference electrode and a working or counter electrode, on which the binding reactions occur, can be measured using the potentiometric sensing technique. A conductometric sensor measures the conduction based on sensing the impedance, potential charges or current, or field effect transduction. In an amperometric sensor a reference electrode is used and is sustained at a fixed electrical potential. Reduction or oxidation of redox groups at the electrode surface creates a transient current flow that has a linear relationship with the concentration of the redox groups. Measuring this current can determine the electrical conductivity of the target molecule. A Faradaic impedance measurement process monitors the electric charges transferred across the reduction-oxidation reaction by the binding of the biomolecules at the electrolyteelectrode interface.

Practically, based on the type of the reference electrode, electrochemical biosensors could carry long term stable measurements, resulting in more accurate readings and enhanced reliability [126-129]. However, the sensitivity of the electrodes also has a significant effect on the sensitivity of the readings. Any presence of impurities in the liquid can also alter the measurements. Moreover, redox markers in the solution are required to be present for any electrochemical measurements [130-132]. 


\section{f) Electronic Detection}

The biosensors designed based on the electronic detection methods are in general a close relative of electrochemical biosensors. They both make use of the electrical properties of DNA sequences. A DNA structure can effectively transfer electric charges through itself with a reasonable current, which is much lower compared to metals [133]. This property makes a DNA sequence suitable for electron-hole transfer, similar to that in semiconductors. However, longer DNA sequences demonstrate slower charge transfer rates.

\section{g) Nanotechnology Based Sensors}

Advances in nanotechnology have assisted researchers to create very small size biosensors which are capable of highly sensitive label-free real time biological measurements [134-136]. A very popular area of research for nanoscale biosensing is biotin modified silicon nanowires (SiNWs) [137-142] to detect sreptavidin at concentrations as low as picomolar. A high surface to volume ratio and very high conductivity enable nanowires and nanotubes to reach ultra high reading sensitivity [143146]. However, the production of an array of nanowires or nanotubes is currently very expensive and involves a complicated fabrication process.

\section{h) Microring Resonators}

A recent area of research in the chemical sensing, and in particular biosensing is the use of optical microring resonators. The analytes floating in the aqueous solution adjacent to the sensor are attracted to the antibodies attached to the sensor surface. The 
microring's evanescent field interacts with the analytes near the sensing surface. The wavelength of the light passing through the microring shifts as it is coupled by the ring resonator. Examining the shift in the light's wavelength can determine the presence of the analytes [147-148]. Different biological molecules cause different resonant responses. Therefore, by using a non-exposed microring as a reference a microarray of rings can detect a wide range of analytes, since each ring would resonate uniquely to the analyte being sensed [149]. Owing to their high sensitivity and selectivity, and also relatively simple fabrication, optical microring resonators are gaining growing popularity among researchers.

\section{i) Field Effect Detection}

As it was pointed out earlier, a DNA sequence has a negative electric charge on its backbone due to the presence of the phosphate groups in aqueous environment. Sensing the electric field generated by the DNA sequence's negatively charged backbone can spot the existence of the DNA molecule. An ion sensitive field effect transistor (ISFET) is an ideal biosensor for detection of DNA sequences taking advantage of their ionizability property.

An ISFET is fundamentally a metal oxide semiconductor field effect transistor (MOSFET) whose metal gate has been replaced by an electrochemical reference electrode in contact with an electrolyte solution, in which the target molecules to be detected are dissolved. The electric field generated by the DNA molecule's ionization modulates the source to drain current in the ISFET, which in turn can be simply measured [150-159]. A more detailed review of the ISFET technology will be explained in Section 
2.4. The superior sensitivity, accuracy, ease of fabrication, and very small size of the ISFET based sensors make them ideal for biosensing, as they have been already used in design of $\mathrm{pH}$ meters.

Each of the detection methods explained above - both label-based and label-free techniques - is employed in difference applications. Although their sensitivity and selectivity are main factors for their use; the higher reliability and reproducibility the techniques have, the more practical biosensors they will be.

\subsection{Transistor}

\subsubsection{MOSFET Basics}

Transistors on the whole are categorized into bipolar and field effect devices. In the context of this thesis only field effect transistors are being reviewed. A simple field effect transistor, most remarkably a MOSFET, is a three terminal device. The terminals are the device's external connection. In this case, they gate, source, and drain which are modified, or doped, regions of the silicon crystal wafer, namely bulk or substrate. Source and drain regions are separated by channel, which in turn is neighbouring the gate. The channel is situated within the substrate.

In order for a MOSFET to operate, there should be a current from drain to source through the channel. The gate terminal is isolated from the channel, i.e. the whole device, through an insulating layer, normally silicon oxide. Applying any charges on the gate polarizes the silicon oxide region, and induces the charge distribution on the surface of 
the semiconductor device. Therefore any changes in the charge distribution at the gate cause the drain to source current, IDS, to change. As it can be seen, a MOSFET is used to convert a small input signal, such as a change in the charge distribution, to a large output signal, current flow.

In the process explained above, it is assumed that the substrate is absolutely isolated electrically from the external area and there is no previous charge distribution in the substrate. Any ambient interference on substrate will hamper the carrier transfer in the channel, within the substrate. In order to avoid such interference, most MOSFETs have a fourth external connection, which is the substrate connection; making them four terminal devices. A simple model of a four terminal MOSFET is presented in Figure 2.12. The effect of presence of charges at the gate on the carrier charge distribution of the channel can be seen in Figure 2.13.

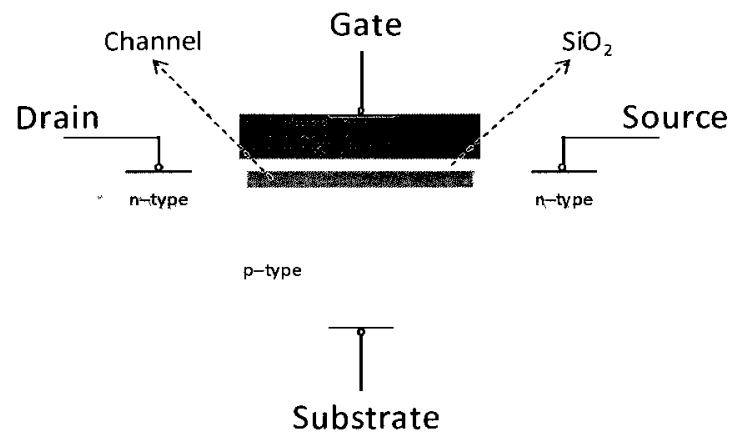

Figure 2.12 - A simple four terminal MOSFET. 


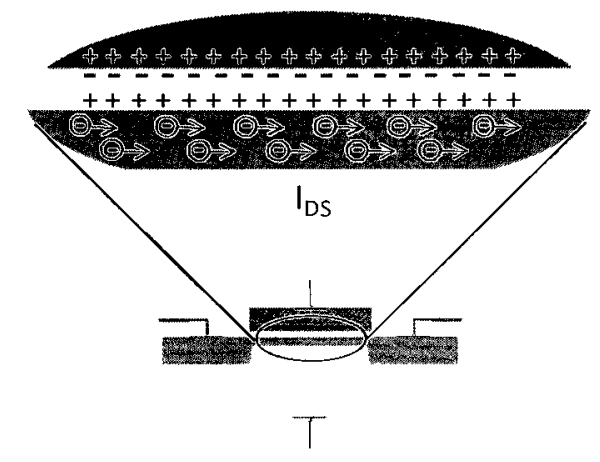

Figure 2.13 - Charge distribution in the conducting channel region of a MOSFET.

\subsubsection{MIS Structure}

The heart of a MOSFET is its gate, and in particular its oxide insulator layer. A metal gate separated from the semiconductor region with an oxide insulator layer is a metal insulator semiconductor (MIS) structure. Properties of a MIS structure, shown in Figure 2.14, will be discussed in this section.

Assuming ideal conditions, the work function (minimum required energy to remove an electron in vacuum from its initial Fermi level) in the metal layer is equivalent to that of the semiconductor layer. However, in the real circumstances, these work functions will not be equal, and a net voltage potential called flatband voltage, $V_{F B}$, is required to apply to the system to create the equilibrium state. If there is no voltage applied across the system, the entire structure is in equilibrium and the Fermi level across the metal and semiconductor are equal. 


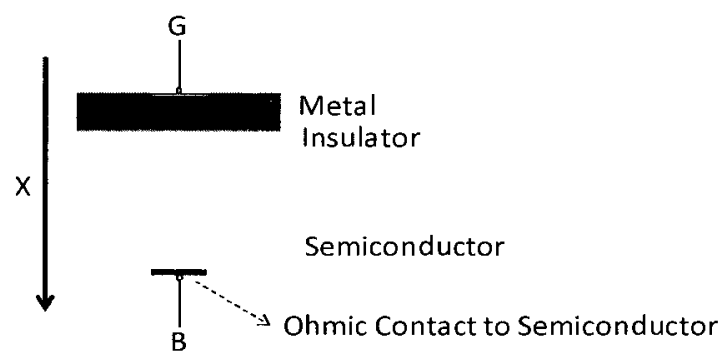

Figure 2.14 - One dimensional view of a MIS structure.

Assuming the semiconductor is set to $0 \mathrm{~V}$, if a voltage potential is applied to the metal with respect to the semiconductor, the equilibrium state is disrupted and the Fermi levels of both materials alter by the same amount, equal to the applied potential difference, but in the opposite direction as shown in Figure 2.15.a). The structure now acts like a capacitor, and the insulator as the dielectric, with charges appearing in the semiconductor-insulator and metal-insulator interfaces.

Assuming the semiconductor is $\mathrm{p}$-type silicon, if a negative voltage with respect to the semiconductor is applied to the metal, the created electric field accumulates the electrons in the metal-insulator interface, leaving behind the holes in the semiconductorinsulator interface. There is no excess charge in the surface of metal or semiconductor, given that the structure is a closed loop system. Since the density of the majority carriers (holes) at the semiconductor-insulator interface increases, the Fermi level of the semiconductor shifts closer to the valence band. The MIS is namely in accumulation state, as seen in Figure 2.15.b). 


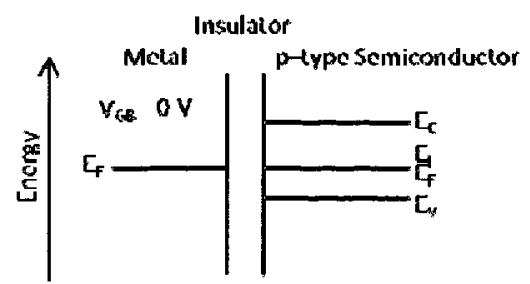

(a)

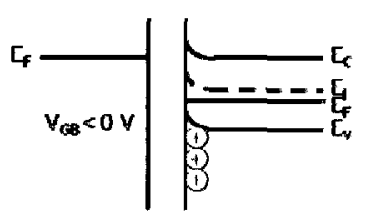

(b)

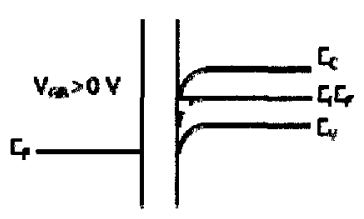

(d)

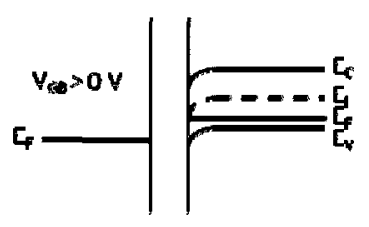

(c)

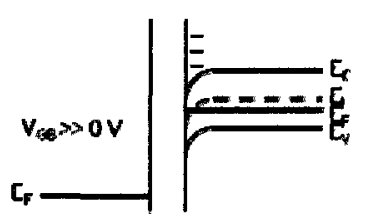

(e)

Figure 2.15 - The Energy bands of a p-type MIS structure as a function of applied voltage, $\mathrm{V}_{\mathrm{GB}}$, in (a) Equilibrium; (b) Accumulation; (c) Depletion; (d) Deep Depletion; and (e) Inversion states.

Likewise, applying a positive voltage potential with respect to the semiconductor onto the metal the majority carriers, electrons, are pushed towards the semiconductorinsulator interface. The Fermi level of the semiconductor at very close to the interface at this phase, depletion, moves closer to the conduction band (Figure 2.15.c)). Additional 
increase of the applied positive voltage potential will create a state in which the concentrations of electrons and holes close to the interface equal each other. This situation shifts the Fermi level back to the middle of the conduction band and valence band, same point where it was before any voltage was applied as shown in Figure 2.15.d). If the positive voltage potential is increase further, a surplus density of electrons will be created in the semiconductor-insulator interface. This situation will transform the lattice in the region very close to the interface into n-type. The MIS will in the inversion state, (Figure 2.15.e)).

The number of created charges, $Q_{s}$, has a direct relationship with the surface potential, $\psi_{s}$, and can be described by Gauss' Law and Poisson's equation:

$$
\frac{\partial^{2} \psi}{\partial x^{2}}=-\frac{q}{\varepsilon_{S}}\left(N_{A}-N_{D}+n-p\right)
$$

where $N_{A}, N_{D}, n$, and $p$ are respectively the concentrations of donor atoms, acceptor atoms, holes, and free electrons, and $\varepsilon_{s}$ is the permittivity of silicon.

\subsubsection{Field Effect Transistors}

As Figure 2.16 illustrates, a field effect transistor (FET) is in principle a MIS capacitor with two regions surrounding the inversion region and doped with a doping opposite to that of the semiconductor. For instance, in a p-type semiconductor FET, the neighbouring regions, Source and Drain, are n-type. An FET with a p-type substrate is called an $n-F E T$, and an FET with an n-type substrate is called a $p-F E T$. 


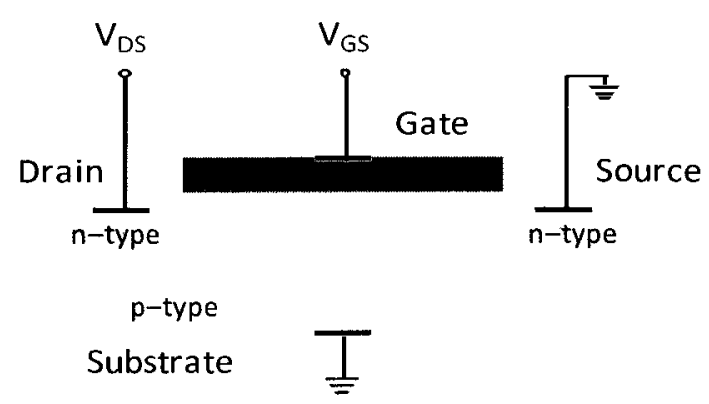

(a)
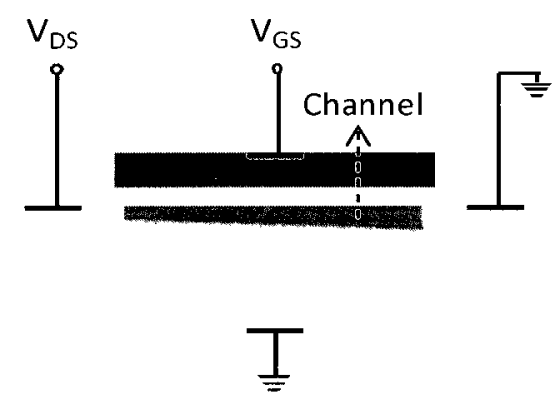

(c)
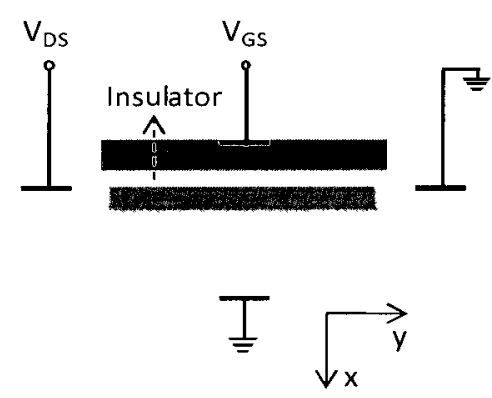

(b)
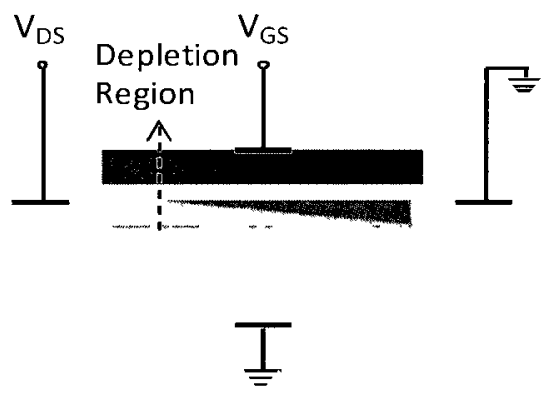

(d)

Figure 2.16 - A p-FET in different states: (a) Sub-Threshold, $V_{G S}<V_{T}$; (b) $V_{G S}>V_{T}$, $\mathrm{V}_{\mathrm{DS}}<\mathrm{V}_{\mathrm{T}}$; (c) $\mathrm{V}_{\mathrm{GS}}>\mathrm{V}_{\mathrm{T}}, \mathrm{V}_{\mathrm{DS}}<\mathrm{V}_{\mathrm{GS}}-\mathrm{V}_{\mathrm{T}}$; (d) $\mathrm{V}_{\mathrm{GS}}>\mathrm{V}_{\mathrm{T}}, \mathrm{V}_{\mathrm{DS}}>\mathrm{V}_{\mathrm{GS}}-\mathrm{V}_{\mathrm{T}}, \mathrm{V}_{\mathrm{DS}}>\mathrm{V}_{\mathrm{DSsat}}$

In typical FET operation, source and substrate are maintained at $0 \mathrm{~V}$, while drain is kept at a fixed voltage of $V_{D S}$, with respect to the source. Assuming all ideal conditions, similar to a MIS, there are no excess minority carriers in the channel region between source and drain. In non-ideal case, similar to the MIS structure, due to various factors such as the gate material, substrate doping, the semiconductor, fixed charged density, 
there is a net charge present within the insulator or in the insulator-semiconductor interface. In order to remove the effect of these charges (i.e. there is no band bending in the semiconductor), a net voltage, flatband voltage, is required across the device.

Applying a negative voltage on the gate with respect to the substrate, $\mathrm{V}_{\mathrm{GS}}$, boosts the density of the majority carriers (holes) in the substrate-insulator interface, creating an accumulation state. In the same fashion, applying a positive $V_{G S}$ pushes the region into depletion. Further increase in positivite $V_{G S}$ drives the interface into deep deletion and ultimately inversion states. As the interface enters inversion, the p-type regions very close to the interface commence to invert into n-type. Formation of an n-type inversion layer right adjacent to the substrate-insulator interface and between source and drain makes an n-type conducting channel linking source and drain together, since all three regions are in fact from the same type. Yet again, a depletion region alongside the channel-substrate interface is being formed, which grows in depth by the increase in $V_{D S}$. The potential at which the FET enters inversion and the conducting channel begins to form is called the threshold voltage, $V_{T}$. The threshold voltage is highly dependent on flatband voltage of the device. The region of operation of the FET below the threshold voltage is called the sub--threshold region.

An FET's generated current, IDS, is dependent on various parameters, such as: $V_{T}$, $V_{D S}, V_{G S}$, and the electrical resistance of the conducting channel. In order to understand this relationship, one must look into the charges at the interface inversion layer, $Q_{n}$, first. $Q_{n}$ is the charge generated the insulator layer, which acts like a capacitor dielectric. 
Assuming the insulator is silicon oxide, when enough voltage, $V_{G S}-V_{T}$, is applied across the insulator layer $Q_{o x}$ is generated:

$$
Q_{o x}=-C_{o x}\left(V_{G S}-V_{T}\right) W L
$$

where in a sample device such as the one presented in Figure 2.17,W and $L$ are, the effective width and length of the channel region (not those of the insulator layer, but the inversion layer), respectively. Also $C_{o x}$, the insulator's capacitance per unit area is:

$$
C_{o x}=\frac{\varepsilon_{o x}}{t_{o x}}
$$

where $t_{o x}$ is the thickness of the silicon oxide insulator layer, and $\varepsilon_{o x}$ is its permittivity. The voltage difference between source and drain create an electric field which the majority carriers, electrons, with a mobility $\mu_{n}$ have to flow through the inversion layer with a length $L$. The channel act as a resistance and the current $I_{D S}$ is generated by such a flow that is linearly proportional to $V_{D S}$ :

$$
I_{D S}=\frac{-\mu_{n} Q_{n}}{L} \frac{V_{D S}}{L}
$$

which gives:

$$
I_{D S}=\mu_{n} C_{o x} \frac{W}{L}\left(V_{G S}-V_{T}\right) V_{D S}
$$




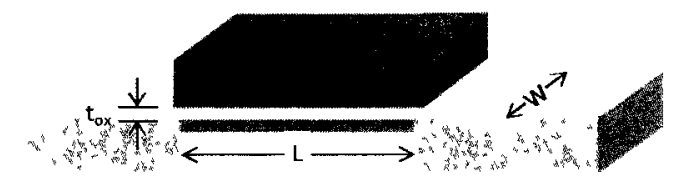

Figure 2.17 - A 3-D view of a FET.

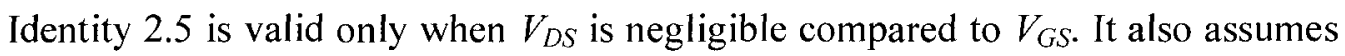
the depletion charge is negligible. When $V_{D S}$ is increased, the depletion region around the drain is enlarged; thus the voltage difference between gate and the depletion region neighbouring drain and the channel is reduced. Therefore, the thickness of the inversion layer in this region is reduced. A non uniform inverse layer means $V_{D S}$ distributes homogeneously in order to drive $\mathrm{I}_{\mathrm{DS}}$ constant in steady state. If factor $\alpha$ represents the quantity of depletion charge (which is normally equal to one), presuming the voltage at point $y$ anywhere along the channel is half of $V_{D S}$ times $\alpha, I_{D S}$ will be:

$$
I_{D S}=\mu_{n} C_{o x} \frac{W}{L}\left(V_{G S}-V_{T}-\frac{\alpha V_{D S}}{2}\right) V_{D S}
$$

Identity 2.6 holds as long as $V_{D S}$ is smaller than the initial voltage difference across the insulator layer, $V_{G S}-V_{T}$, considering $\alpha$ factor. When $V_{D S}$ exceeds this limit, there will be a point where the electric field between drain and source is not enough to expand the depletion region. The reduction of the inversion layer discontinues, or in other words. Hence, increasing $V_{D S}$ can no longer affect $I_{D S}$ and it saturates. $I_{D S}$ for $V_{D S}$ at or above saturation, $V_{D S \text { sat }}$, is: 


$$
I_{D S}=\mu_{n} C_{o x} \frac{W}{L}\left(V_{G S}-V_{T}-\frac{\alpha V_{D S s a t}}{2}\right) V_{D S ~ s a t}
$$

where

$$
V_{D S \text { sat }}=\frac{V_{G S}-V_{T}}{\alpha}
$$

therefore:

$$
I_{D S}=\mu_{n} C_{o x} \frac{W}{L} \frac{\left(V_{G S}-V_{T}\right)^{2}}{\alpha 2}
$$

Figure 2.18 shows the unsaturated (linear) and saturated regions of $V_{D S}$ as a function of the applied gate voltage, $V_{G S}$.

The above conditions are met only if it is assumed:

1. There are no interface traps, fixed oxide charge, or work function difference

2. The inversion layer is doped homogeneously.

3. Carrier mobility along the channel is constant.

4. The electric field across the inversion layer is negligible compared to the electric field along it.

5. Diffusion and leakage current are negligible. 


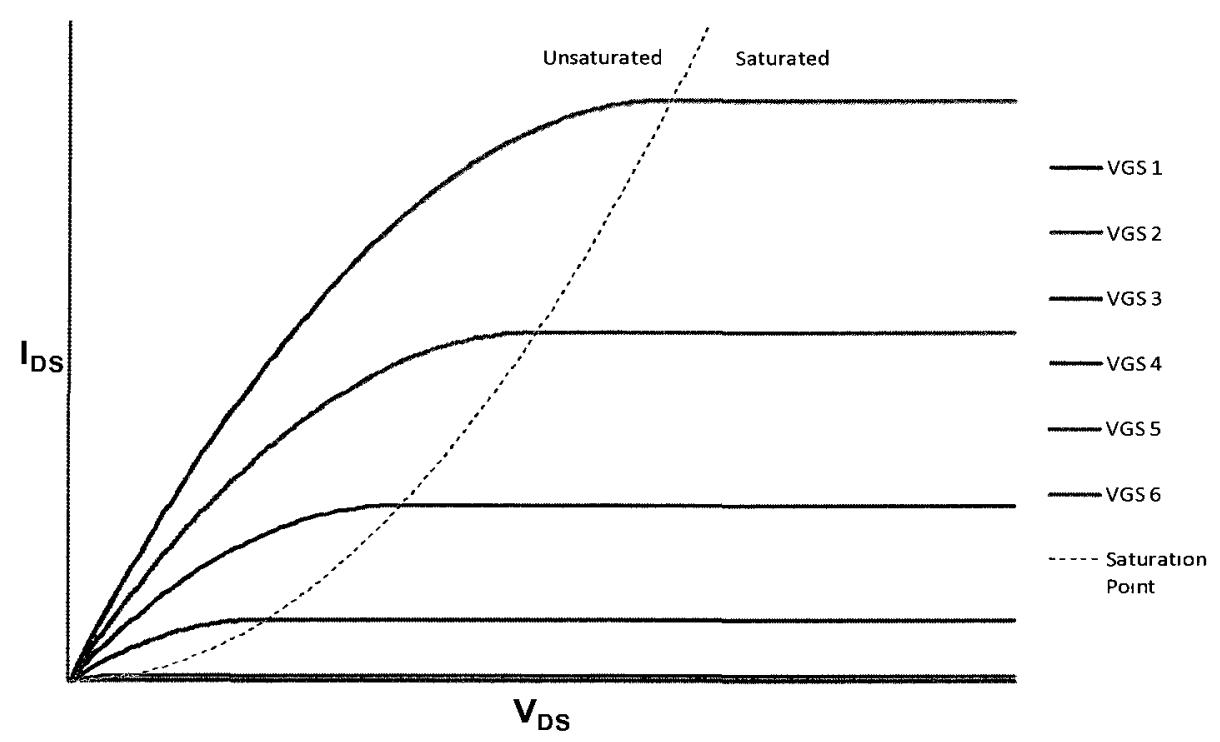

Figure 2.18 - The $I_{D S} v s . V_{D S}$ plot showing the unsaturated and saturated regions for different values of $\mathrm{V}_{\mathrm{GS}}$ and $\alpha=1$.

For more details about the semiconductor's physics, MIS structure and FET operation, the reader is encouraged to refer to [160-161].

\subsection{Ion Sensitive Field Effect Transistors}

A FET can detect any small voltage or charge changes on its gate and convert them into measureable current changes on $I_{D S}$. The charge change can be generated by chemical reactions in an aqueous solution, electrolyte, with direct contact to the surface of a catalytic metal gate. Biasing the gate, through the electrolyte, is possible by using a reference electrode placed in the solution.

If the metal gate is replaced with a chemically sensitive layer, whose charge distribution attributes change when placed in contact with the electrolyte, then a voltage 
potential of $V_{G S}$ is established across the insulator with respect to the reference electrode. The generation of negative and positive ions in the solution create enough charges which in turn can modify the charge distribution in the gate surface, and consequently the properties of inversion layer of the FET. Such a device is an ion-sensitive field effect transistor (ISFET), and highly sensitive to the $\mathrm{pH}$ changes in the solution. This property has made electronic $\mathrm{pH}$ sensors possible.

Figure 2.19 shows an ISFET in contact with an electrolyte solution. For the purpose of encapsulating the other surfaces of the sensor excluding the measurement site silicon nitride, $\mathrm{Si}_{3} \mathrm{~N}_{4}$, is an ideal insulator since unlike silicon dioxide, it does not have open ring structure, and is less permeable. Moreover, silicon nitride has a denser structure than silicon dioxide.
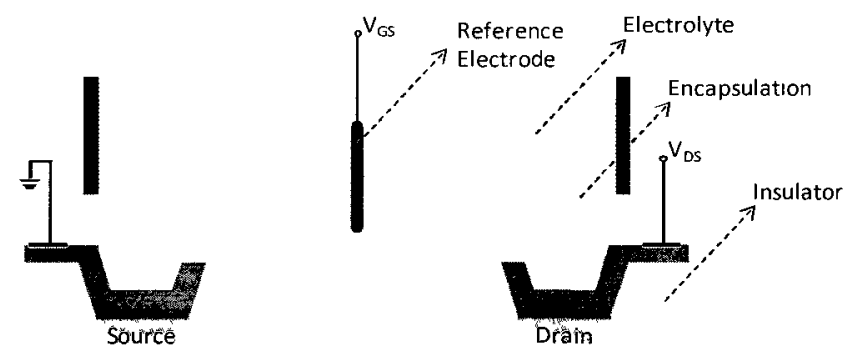

Substrate

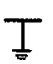

Figure 2.19 - A general schematic of an ISFET.

The exposed insulator layer of the gate can also sense charge in the electrolyte; however, the insulation characteristic of the layer is reduced because of the hydration of the ISFET's insulator, normally a layer of thermally grown $\mathrm{SiO}_{2}$. At the surface of the insulator, the functional $-\mathrm{OH}$ groups react with the hydrogen ions of the electrolyte 
solution. Both groups are able to accept or donate a charge. The following reactions are the outcomes:

$$
\begin{aligned}
& \mathrm{Si}-\mathrm{OH}_{2}^{+} \leftrightharpoons \mathrm{Si}-\mathrm{OH}+\mathrm{H}^{+} \quad \ldots \quad K_{1}=\frac{[\mathrm{Si}-\mathrm{OH}]\left[\mathrm{H}^{+}\right]}{\left[\mathrm{Si}-\mathrm{OH}_{2}^{+}\right]} \\
& \mathrm{Si}-\mathrm{OH} \leftrightharpoons \mathrm{Si}-\mathrm{O}^{-}+\mathrm{H}^{+} \quad \ldots \quad K_{2}=\frac{\left[\mathrm{Si}-\mathrm{O}^{-}\right]\left[\mathrm{H}^{+}\right]}{[\mathrm{Si}-\mathrm{OH}]}
\end{aligned}
$$

These reversible reactions occur until a stable equilibrium is established. For low values the equilibrium constant, of $\mathrm{K}_{1}$ and $\mathrm{K}_{2}$, the surface will not behave in a Nernstian manner towards the hydrogen ions. The reactions 2.10 and 2.11 indicate a change in charge distribution of the insulator surface due to the presence of the electrolyte solution. The change in the charge distribution of the insulator surface in return causes a shift in the flatband voltage of the ISFET, resulting in a change in the threshold voltage of the device and the current output of the transistor. For a p-type ISFET the higher the number of hydrogen ions are present, i.e. the smaller the $\mathrm{pH}$, the more the charge distribution creates positive charge at the surface, which reduces the transistor's output current. However, in an n-type transistor, a larger population of hydrogen atoms denotes a more negative charge distribution at the insulator surface, resulting in a larger output current.

An ISFET biosensor measures the threshold voltage before and after a chemical or biological reaction take place in the measurement region, and compares the two. The threshold voltage difference is interpretable from the change in the transistor's current due to the chemical reaction. Taking the derivative of equation 2.9 and rearranging it will result in: 


$$
\Delta V_{T}=-\frac{\left(V_{G S}-V_{T}\right)}{2} \frac{\Delta I_{D S}}{I_{D S}}
$$

The electrolyte plays a major role in establishing charges in an ISFET. In general, an electrolyte is a buffer solution in which both positive and negative mobile ions are present in nearly equal numbers. Therefore, an electrolyte behaves similarly to a semiconductor when it is under the effect of an electric field, it creates its own polarized regions. The electrical properties of an electrolyte are described by its Debye length. Debye length is the distance in the electrolyte from a reference, working or counter electrode, beyond which the electric field of a charged particle, ion, is shielded by particles with opposite sign charges. Thus, the influence of a reference, working or counter electrode in the electrolyte extends approximately a Debye length into the solution.

A higher concentration of ions in the electrolyte gives a larger voltage potential drop at an electrolyte surface. However, the electrolyte's Debye length in higher concentration is shorter. Therefore, if an electrolyte is gating an ISFET (i.e. biasing the transistor's gate) due to the effects of Debye length there is a voltage drop on the regions close to the transistor's gate, reducing the effective $V_{G S}$. In turn, an electrolyte with lower concentration of ions results in a smaller transistor output current. Nevertheless, at the high concentrations of electrolyte, a higher local charge distribution is generated at the surface of the gate, compared to low concentrations. This means the effective $V_{G S}$ of the ISFET is larger at high electrolyte concentrations in contrast to low concentrations, producing a larger $I_{D S}$. This phenomenon is illustrated in Figure 2.20. 


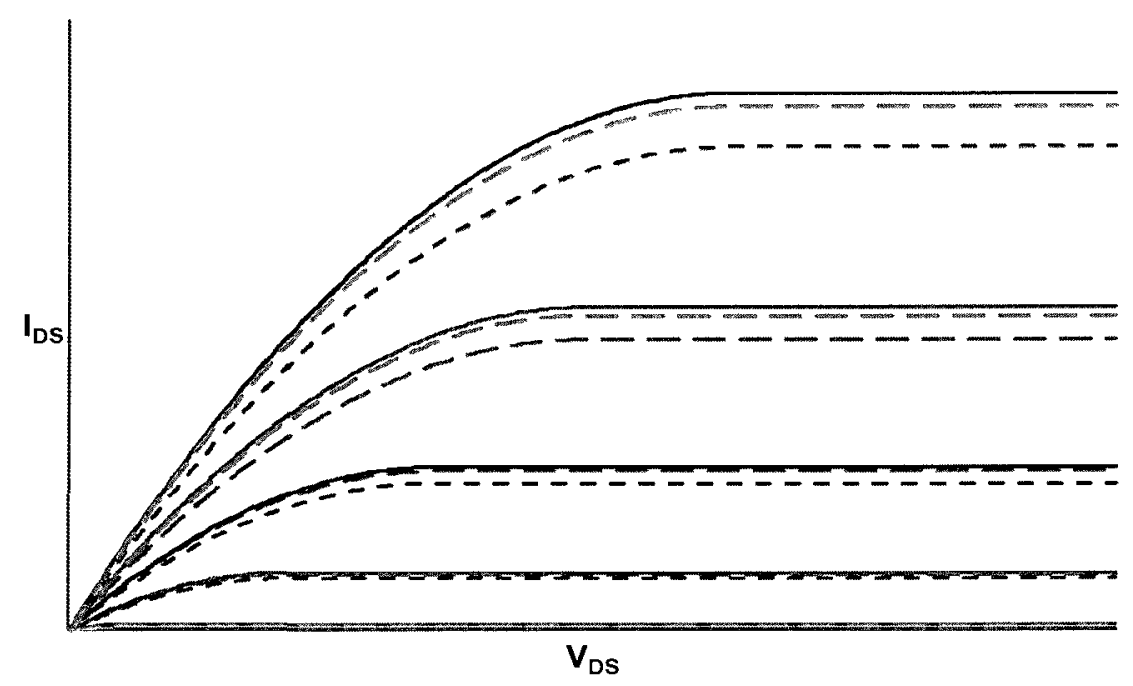

Figure 2.20 - $\mathrm{I}_{\mathrm{DS}}$ current $v s . \mathrm{V}_{\mathrm{DS}}$ in a MOSFET (solid) compared to the $\mathrm{I}_{\mathrm{DS}}$ of an ISFET with $1 \mathrm{mM}$ (dashed) and $0.1 \mathrm{M}$ (long dashed) electrolytes. $\mathrm{V}_{\mathrm{GS}}$ in the ISFET is applied through a reference electrode. Each colour represent a unique $\mathrm{V}_{\mathrm{GS}}$.

It is assumed that the concentrations of negative and positive ions of the electrolyte are at a steady equilibrium at all times. Any small change in the concentration equilibrium impacts the concentration of the hydrogen ions at the insulator surface, which in turn creates a very large change in the voltage of the ISFET, and consequently the output current of the transistor. This reduces the stability, accuracy, and selectivity of the ISFET based biosensor.

More information about the ISFETs operation and their surface chemistry can be found at [162-168]. 


\subsection{DNA BioFET}

An ISFET can be a DNA sequence detector, or a DNA bioFET, if the sequence is immobilized at the surface of the gate insulator, or the surface is functionalized. Placing the DNA molecule at the insulator surface, introduces negative charges to the insulator, and changes the charge distribution. Assuming DNA molecules are immobilized homogeneously, a monolayer of DNA sequence molecules can be considered as a uniformly charged membrane, where the thickness of the membrane is the length of the DNA molecule, normally approximately $10 \mathrm{~nm}$, or about 30 base pairs.

If the immobilized molecules are identical ssDNA sequences, introducing their non-complementary sequences into the electrolyte will not change the charge distribution of the DNA membrane, or the insulator, since the two strands will not react or hybridize. Conversely, the complementary sequences hybridize and the resulting dsDNA sequences posses more negative charges, which modifies the insulator's charge distribution. As a result, if the bioFET is a p-Type transistor, introducing ssDNA sequences, and then hybridizing them increase the bioFET's output current at both stages, whereas no change will be seen in the bioFET output current when non-complementary sequences are introduced. This effect is shown in Figure 2.21.

A ssDNA sequence or a hybridized dsDNA sequence tends to have locations that can ionize and produce $-\mathrm{OH}$ groups. Such locations are called binding sites. The $-\mathrm{OH}$ groups created through binding have a tendency to temporarily react with the positively charged ions in the electrolyte. Not only do they slightly increase the $\mathrm{pH}$ of the solution, 
but also they change the charge distribution at the insulator surface, increasing the flatband voltage of the transistor, and thus noticeably affecting the sensitivity of the bioFET. For this reason, the lower the density of binding sites present at the DNA sequence is, the higher the sensitivity of the bioFET will be [38], [169-170].

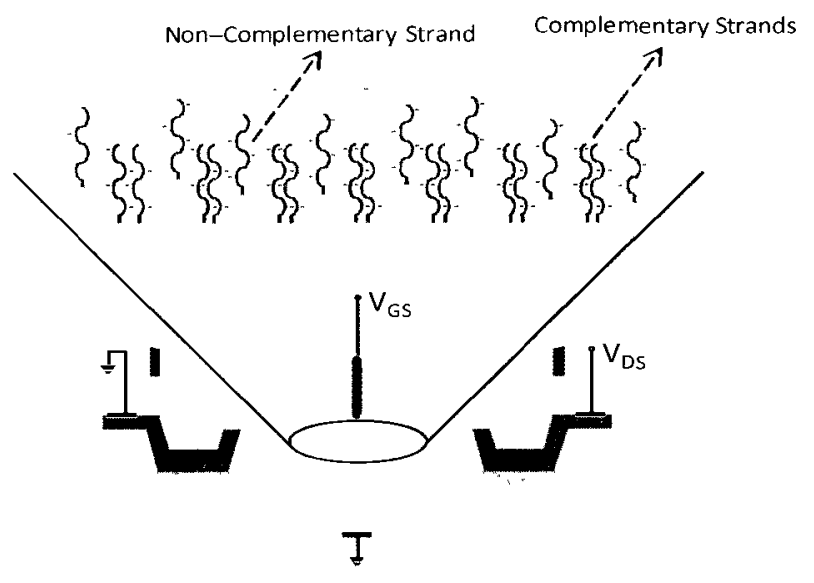

Figure 2.21 - DNA functionalization and hybridization on a BioFET.

When the ssDNA molecules are immobilized at the sensor surface, they ionize at the electrolyte. These negatively charged DNA molecules generate an electric field around them. Although the electric field is stronger at the electrolyte regions close to the DNA molecule, it is dominated by the channel. Hence, it effects the charge distribution at the interface between the device channel and the insulator through the gate. The presence of the ssDNA effectively results in an increase in the charge distribution at such interface, resulting in larger threshold voltage and output current of the bioFET sensor. The immobilized molecules do not cover all the available binding sites at the sensor surface. Therefore, the device is still sensitive to the changes in the concentration of the ions in 
the electrolyte through the remaining available binding sites. If one monitors the threshold voltage changes of the devices because of the changes in the electrolyte concentration both before and after immobilization, it can be concluded that such threshold voltage changes would increase because of the DNA immobilization process.

In the same fashion, the DNA hybridization increases the charge negativity of the entire molecule, and therefore the charge density at the channel-insulator interface. This is expected to cause a further increase in the threshold voltage, i.e. the output current of the device. However, since the entire hybridized molecule has a larger negative charge than before hybridization, the electric field due to this charge increases the charge screening by the electrolyte. Therefore, the increase in the threshold voltage of the device in response to the hybridization process is not as large as the increase due to the immobilization process. This effect is more noticeable when one considers the changes in the threshold voltage in response to the changes in the electrolyte concentration, rather than monitoring the absolute changes of the threshold voltage [1-4].

DNA hybridization at high electrolyte concentration is generally quicker and more effective than at lower concentrations. However, as mentioned earlier, at very high electrolyte concentrations, the Debye length is much shorter. This effect reduces the sensitivity of the bioFET. On the other hand, at very low concentrations the solution's $\mathrm{pH}$ is unstable. Therefore the sensing operation is advantageous at an optimum concentration, neither very high, nor very low.

Increasing the sensitivity of the bioFET through increasing the number of binding sites is feasible by various methods such as using hafnium oxide or hafnium silicate 
insulator instead of silicon oxide [5]. While the techniques involving immobilization on an insulator layer are more popular, there is a possibility of increasing the number of binding sites at the sensor surface by DNA immobilization directly on the semiconductor channel. Particularly, if the bioFET is based on a $<111>$ silicon crystal substrate. At the surface of $\mathrm{a}<111>$ silicon lattice the silicon atoms are positioned closer to each other than for the $<100>$ lattice. Therefore, a silicon $<111>$ surface allows creation of more binding sites per unit ear, which can result in a denser DNA immobilization, and in turn higher detection sensitivity [171]. Although research has been done to immobilize DNA molecules onto the silicon $<111>$ crystal directly [172], efforts of DNA immobilization on an intermediate layer have been more successful. There have also been efforts to include a secondary gate, a suspended gate to increase the charge distribution at the sensing surface of the ISFET [173].

Another method to enhance the bioFET sensitivity is coating the silicon oxide insulator layer of the gate with a highly conductive metal, such as gold [142]. A gold coated gate considerably reduces the density of binding sites and allows DNA immobilization through thiol linkers. The gold-thiol bond is very stable and it creates an alkanethiol SAM. The thiolated DNA molecule is essentially a thiol group attached to an oligomer through a 6 -carbon spacer. However, a small number of ssDNA sequences are able attach to the gold surface directly without the thiol linker, since the DNA sequence is a charged molecule and the gold surface adjacent to an ion-rich electrolyte is loaded with surface charges. Given that the DNA-gold bond is unstable in buffered electrolyte solutions (especially in higher concentrations), the directly attached DNA sequences 
separate over time and result in a distortion in the measured signal [174]. Therefore, normally the gold surface of the sensor is washed with high concentration buffer solutions shortly after functionalization and hybridization [175-176].

\subsection{Reference Electrode}

Maintaining a steady equilibrium of positive and negative ions in an electrolyte solution is conceivable only if a stable constant voltage potential is established in the electrolyte. Therefore maintaining such a stable voltage potential by a reference electrode is very important in the stability of the biosensor. Some of the most stable reference electrodes are silver - silver chloride electrodes, which perform by the half cell oxidation - reduction reaction at the surface of the electrode. A customary reaction of a reference electrode is:

$$
\mathrm{AgCl}+\mathrm{e}^{-} \leftrightharpoons \mathrm{Ag}+\mathrm{Cl}^{-}
$$

which produces or consumes chloride ions of the electrolyte.

The concentration of chloride ions controls the ionization of the other ions in the electrolyte [177]. Therefore, the reference electrode itself introduces an additional work function to the bioFET sensing system, which should be taken into account when determining the threshold voltage of the transistor. The work function of a typical commercially available $\mathrm{Ag}-\mathrm{AgCl}$ reference electrode can be verified by the Nernst equation of chloride ion at room temperature: 


$$
E=E^{0}-0.05916 \log \left(\frac{1}{\left[C l^{-}\right]}\right)
$$

where $E^{0}$, the standard cell potential, at room temperature for a $1 \mathrm{M}$ concentration of chloride ions is $222.34 \mathrm{mV}$. Most of the commercially available $\mathrm{Ag}-\mathrm{AgCl}$ reference electrodes have very low work function at ionization equilibrium [178]. However, all reference electrodes are inclined to drift slowly over time. This drift alters the work function of the electrode and reduces the sensitivity of the bioFET sensor. 


\section{Chapter 3}

\section{Fabrication}

The bioFET sensors were made through CMOS compatible fabrication and postprocessing steps, respectively at Carleton University's Microelectronics Fabrication Laboratory and National Research Council of Canada's Nanofabrication Centre at Institute of Microstructural Sciences. The layout design of the devices (produced by Ryan Griffin) is shown in Figure 3.1.

\subsection{Device Fabrication}

The bioFET sensors are no more than ion sensitive field effect transistors. In order to build a bioFET sensor, one must fabricate a field effect transistor and subsequently post-process it into an ISFET. The bioFETs used in this project were essentially $50 \mu \mathrm{m} \times$ $50 \mu \mathrm{m}$ long channel P-FET devices fabricated on approximately $5 \Omega$-cm bulk $<111>$ silicon crystal wafers. The choice of silicon $<111>$ based crystals over conventional $<100>$ was since overlapping projects, such as direct silicon immobilized bioFETs, required $<111>$ substrate. $<111>$ structure offers a higher density of silicon atoms at the surface of the crystal, which creates a higher number of bonding sites available to attract more DNA molecules. Therefore, a higher surface sensitivity in a $<111>$ crystal is expected than a $<100>$ lattice. Although this feature is not available to the devices used in this work, the overlapping projects made use of this feature. And since such projects 
shared the common fabrication process, the bioFET sensors of this work were also fabricated on $<111>$ silicon wafers.

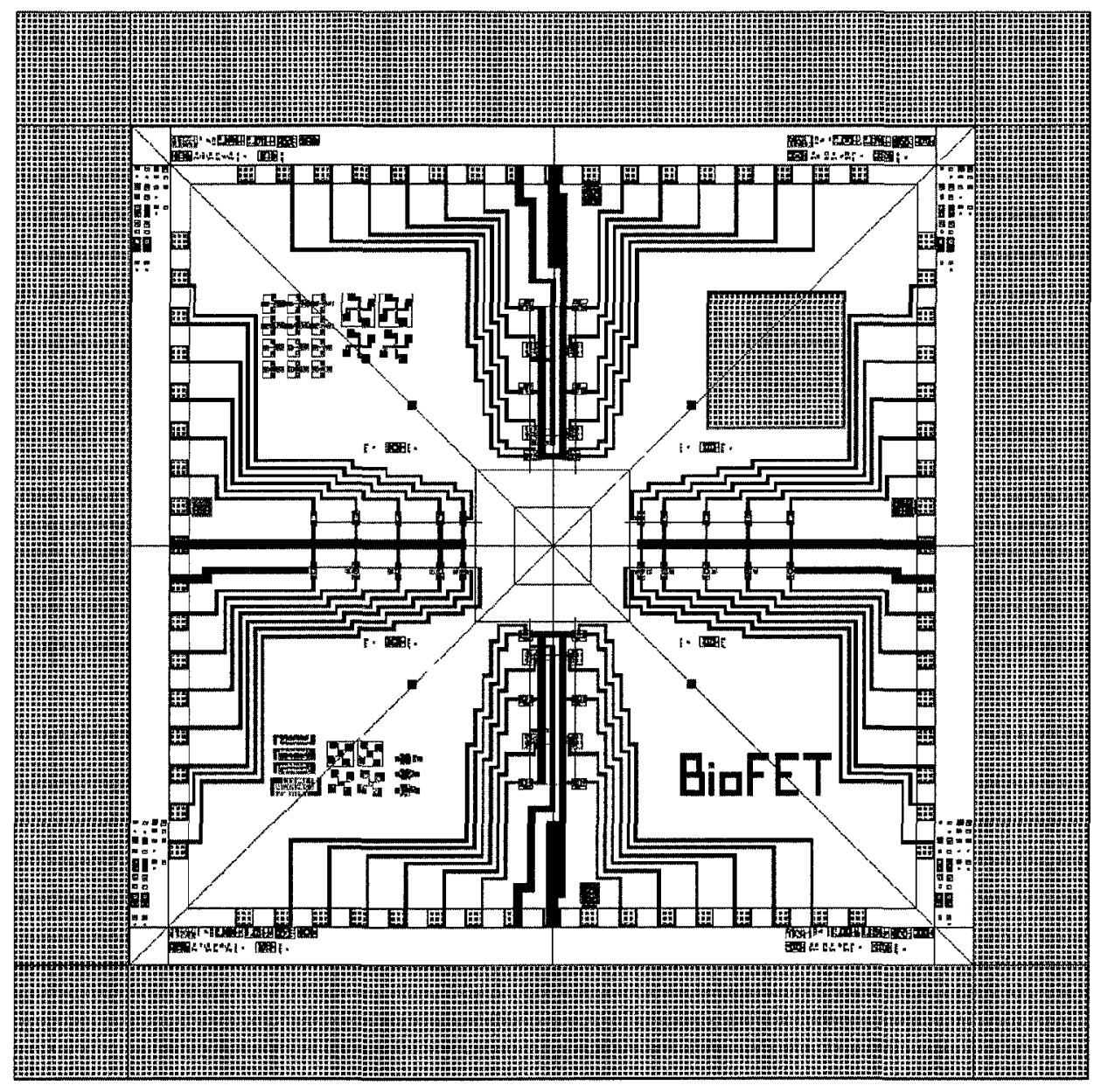

Figure 3.1 - Layout design of the bioFET devices. Devices under test are shown in the middle arrays on both sides of the chip.

The fabrication process (performed by Rob Vandusen and Carol Adams) presented in Figure 3.2 consisted of device isolation, gate and source and drain junctions formation, and ultimately metallization. 


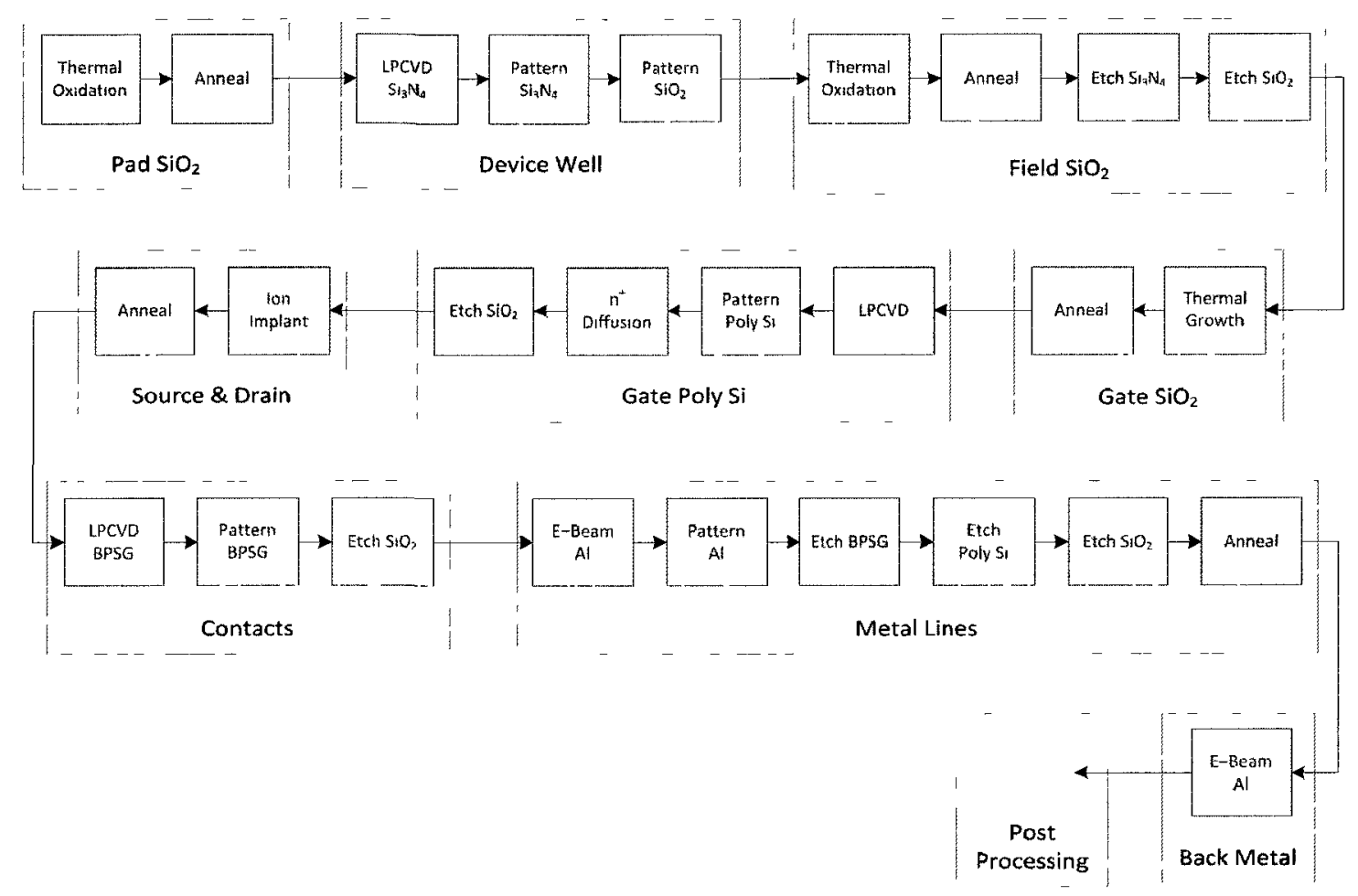

Figure 3.2 - Process flow diagram of the bioFET fabrication process.

\subsubsection{Device Isolation}

Interference from the nearby devices in an array can significantly degrade the performance of a bioFET. In particular, a leakage (through an electric field or a charge transfer) between the active regions of neighbouring FETs at the silicon substrate can considerably affect the characteristics of each bioFET sensor. Therefore, a well suited isolation is needed to cut off any interactions between adjacent devices. A popular isolation technique in device fabrication shown in Figure 3.3, which is also widely used at Carleton University's Microelectronics Fabrication Laboratory, is local oxidation of silicon (LOCOS) [179]. 


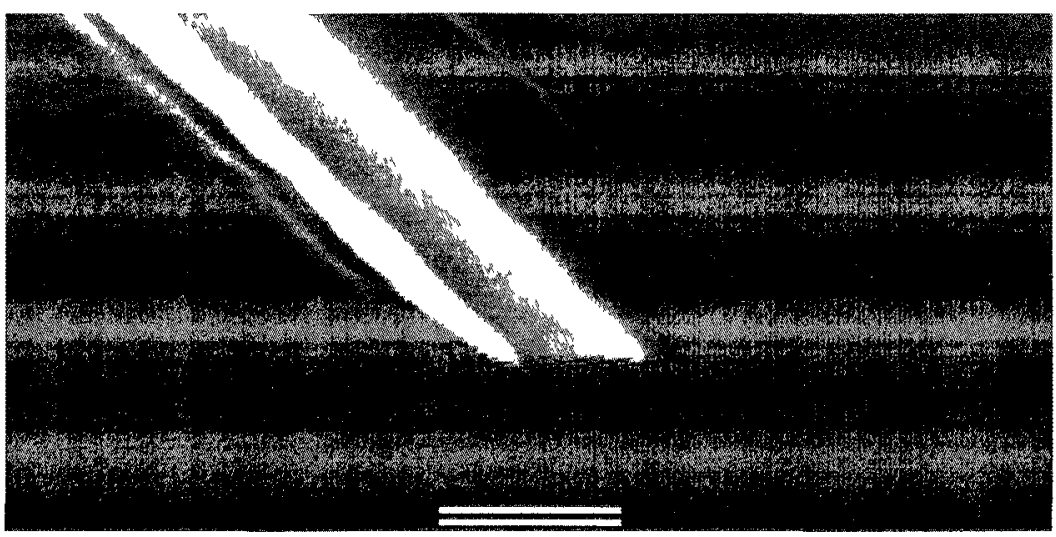

Figure 3.3 - Cross section of typical LOCOS profile fabricated at Carleton University Fabrication Laboratory; The active region is visible in the middle, and the field oxide areas surrounding it.

LOCOS isolation method involves forming a thick layer of silicon oxide, called field oxlde, between the adjacent devices in a way that the active region of each device is bounded by the field oxide. The fabrication process began with growing a thin layer (585 $\AA$ thick) of pad silicon oxide by thermal oxidation in dry oxygen at $1,100^{\circ} \mathrm{C}$ for 11 minutes. A $676 \AA$ thick layer of silicon nitride was then deposited using low pressure chemical vapour deposition (LPCVD) at $820^{\circ} \mathrm{C}$ for 11 minutes, and annealing in forming gas for 20 minutes. The FETs active regions or the device wells were defined by patterning the nitride and oxide layers using plasma etch for 3 minutes, and buffered HF oxide etch (BOE) for 1 minute and 45 seconds, respectively.

The regions covered by the nitride represent the device wells. Conversely, in the exposed areas, a $1.1 \mu \mathrm{m}$ thick field silicon oxide layer was formed by thermal growth in steam ambient at $1,100^{\circ} \mathrm{C}$ for 180 minutes and 40 minutes of anneal in forming gas. To 
expose the active device regions, the nitride and pad oxide layers were then etched in $\mathrm{HF}$ for 2 minutes and 20 seconds, and 1 minute, respectively.

\subsubsection{Gate and Junctions Formation}

The device gate's insulation layer was formed by thermally growing a thin silicon oxide layer with thickness of $543 \AA$ at $1,100^{\circ} \mathrm{C}$ for 11 minutes in dry oxygen, and 40 minutes annealing in forming gas. Since the active device regions' resistance was sufficient for an acceptable threshold voltage, no ion implant process, a common processing step, was required to adjust the threshold.

The polysilicon layer of the gate terminal was fabricated on top of the gate oxide using a self-aligned process through depositing and patterning a $3,770 \AA$ thick layer of polysilicon by LPCVD from silane at $625^{\circ} \mathrm{C}$ for 25 minutes. The patterned polysilicon region was chosen to be larger than the gate area just to ensure it covers the entire gate region, and protects it from subsequent etch processes. In order to ensure the polysilicon layer has low resistance, it was doped for 6.5 minutes by a $10^{15} \mathrm{~cm}^{-2}$ dose of $\mathrm{n}^{+}$ phosphorous by diffusion.

After the poly layer deposition and doping steps, the boundaries of the source and drain regions were defined by exposing the bare silicon crystal. As a result, the gate oxide layer at such areas was removed through 5 minutes and 20 seconds of HF etch. Subsequently, the source and drain junctions were formed through an ion implantation process. This step involved patterning a PR mask and then implanting a $3 \times 10^{15} \mathrm{~cm}^{-2}$ dose of boron with implant energy of $30 \mathrm{KeV}$. The boron implant provides low resistant 
$\mathrm{p}^{+}$regions for source and drain. The $\mathrm{p}^{+}$regions were deep enough that ensure during the e-beam and annealing processes, if the metal layer of the contacts penetrate through the junctions, it would not reach the silicon substrate.

In order to activate the dopant and also remove any damage caused by the ion implant, the wafers were annealed at $1,000{ }^{\circ} \mathrm{C}$ for 5 minutes in forming gas. The temperature and duration of the annealing process was sufficient to remove any implant damages, while it did not excite the boron dopant atoms to penetrate into the device channel. Limiting the penetration of the boron atoms into the channel was very crucial in this step, since any defects in the device channel by the dopant atoms would significantly boost the threshold voltage.

\subsubsection{Metallization}

In order to layout the metal contacts at the surface of the source and drain regions, a local planarization technique was used that consisted of depositing, patterning and etching a layer of boron-doped phospho-silicate glass (BPSG). With only the contact regions exposed, the purpose of the BPSG layer was to protect the rest of the wafer surface from the damages caused by e-beam metallization. The 5,718 $\AA$ thick BPSG layer was deposited by LPCVD at $450^{\circ} \mathrm{C}$, and patterned using etching in buffered dip (BD) etchant for 3 minutes. After patterning the BPSG layer, metallization was done by an e-beam process which formed a $1,086 \AA$ thick layer of aluminum at the surface. The metal lines (and contacts) were patterned by a wet etch process in phosphoric acid at 60 
${ }^{\circ} \mathrm{C}$ for 4 minutes and 20 seconds until the aluminum coating was removed at the undesired areas.

Following metallization steps, the BPSG, and also the polysilicon and silicon oxide layers at the back of the wafers were removed. The BPSG removal involved reactive ion etching (RIE) for 10 minutes. Then the back poly layer was plasma etched in $\mathrm{CF}_{4}-\mathrm{O}_{2}$ for 1 minute and 30 seconds. Finally, the back field oxide was etched using $\mathrm{BOE}$ for 1 minute and 15 seconds. At this point, another annealing step was required to remove any damages caused by the metal e-beam process. This annealing step also considered the duration and temperature in order to prevent the metal diffusing into the substrate, or boron atoms penetrating into the device channel. Therefore a $400^{\circ} \mathrm{C}$ annealing for 10 minutes was done in $100 \%$ nitrogen forming gas.

The final step of the fabrication consisted of metallization for the back of the wafers using an e-beam process forming a $0.25 \mu \mathrm{m}$ thick layer of aluminum.

\subsection{Post-Processing}

Following the fabrication of the bioFETs, they post-processing steps were performed on the chips in order to convert the FETs into suitable ISFET devices. These steps included passivation, gate treatment, device testing, and packaging the chips before the biological experiments in the electrolyte were executed on them. The post-processing steps were primarily done at Nanofabrication Centre, and Surfaces and Interfaces group at Institute of Microstructural Sciences of National Research Council of Canada. These 
steps shown in Figure 3.4, included passivation, gate patterning, surface gold deposition (all performed by Weihong Jiang), device tests, and packaging.

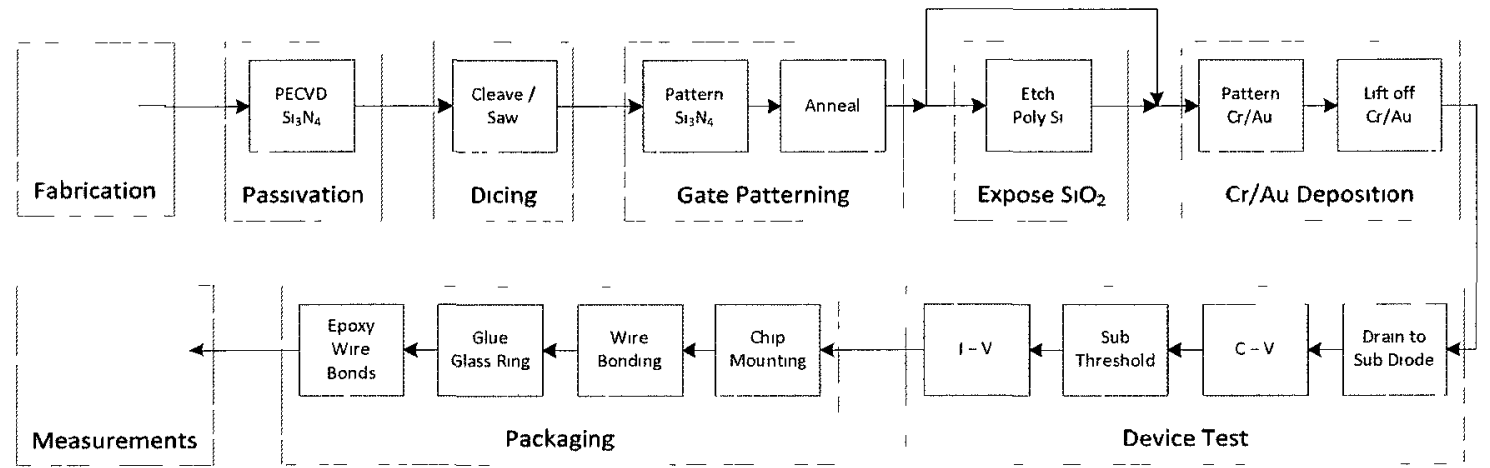

Figure 3.4 - Process flow diagram of the bioFET post-processing steps.

\subsubsection{Passivation}

After the metallization processes, the aluminum metal lines at the surface of the wafers were completely exposed. Therefore, a passivation process was required in order to insulate the metal lines from the electrolyte during the measurement. The passivation layer would also encapsulate the devices from the electrolyte. Silicon nitride was selected as the superior choice of passivation material due to various reasons. First, it is widely used in compatible CMOS processes. Secondly, silicon nitride is an excellent electrical insulator. And above all, unlike silicon dioxide it does not have open ring structure, and is less permeable, and has a denser structure. Therefore, silicon nitride is an appropriate layer acting as a diffusion barrier to any interference in the electrolyte. A plasmaenhanced chemical vapour deposition (PECVD) process with helium dilution formed a $1.2 \mu \mathrm{m}$ thick layer of silicon nitride. 
For the rest of the post-processing, each sample chip would receive unique treatments depending on its individual properties. Hence, each wafer was cleaved and/or sawed into 6 bioFET chips. However, since the wafers were fabricated based on $<111>$ silicon crystals and it was harder to scribe them, cleaving created some physical damage of some of the chips. Particularly, some of the external contact pads were broken at this step which prevented measuring the corresponding devices.

\subsubsection{Gate Patterning}

At this point of the process, the device gates and the external metal contact pads were exposed by removing the nitride passivation layer using Inductively Coupled Plasma (ICP) dry etch in $\mathrm{C}_{4} \mathrm{~F}_{8}$. In order to protect the regions adjacent to the gate area from the wet etch (and subsequent dry etch) and any possible diffusion of the metal deposition in the next step, the exposure area in this etching process was chosen smaller than the region covered by polysilicon and slightly larger $(5 \mu \mathrm{m})$ than the gate area.

Although all the devices on a single chip were treated similarly, surprisingly, the dry etch was not uniform across the chip. It appeared that the selectivity of the etch to the nitride layer was inconsistent and some devices required longer exposure to the etchant. Longer etch process for some devices potentially caused extended lateral etch, which exposed large gate area. Next, the chips were annealed at $450{ }^{\circ} \mathrm{C}$ for 20 minutes in $96 \%$ nitrogen forming gas to reduce the inconsistency. Also, this annealing step was performed in order to ensure the aluminum layer at the contact pads was redistributed and had a smooth surface before depositing a layer of chromium-gold on it. However, it was 
done at relatively low temperature. Longer or higher temperature annealing not only roughens the metal surface, but also it creates the possibility of aluminum diffusing into the source and drain contacts.

While the common process of bonding gold onto FET gates involves removing the polysilicon layer, some of the chips were chosen to have poly left on them. This decision was made since the poly layer is doped with a high dose of $n^{+}$phosphorous dopant and therefore it has a very low resistance. However, the rest of the chips were processed with HF based wet etch for 45 seconds to remove their gate polysilicon layer. Figure 3.5 shows a cross section of the gate area and the adjacent region after the wet etch. As explained above, the region covered by polysilicon was slightly larger than the gate area; therefore, a narrow strip of exposed polysilicon was left around the gate after the wet etch. Although this narrow strip would be covered by a top level metal layer in the next step of the process, since it is very narrow and it expands beyond the area overlapping the device well, its presence would not affect the device channel. Therefore, the presence of this narrow strip would not influence the bioFET sensitivity.

The choice of gold for covering the bioFETs' gate surfaces was due to the fact that it is the most suitable option for bonding thiol groups to the surface of the sensors. Additionally, if the gold layer oxidizes, the thickness of such gold oxide layer would be relatively small. Moreover, the gold surface oxidation is unstable when the surface is exposed to electrolyte. Instead, the floating $-\mathrm{OH}$ ions will be attracted by the surface of the gold layer and create bonding sites. Atomic oxygen cleaning also ensures that gold surface would not include any gold oxide molecules [180-183]. Nonetheless, the 
adhesion of the deposited layer of gold on silicon oxide or polysilicon is not stable and would detach [142], [174]. As a result, a layer of chromium was used to coat under the gold. Chromium was preferred to platinum, palladium, and titanium based on the tests that were performed with these metals and gold; chromium showed the best attachment profile onto both silicon oxide and polysilicon. Moreover, chromium also adheres well to the aluminum layer at the external contact pads. The chromium-gold layer protects the contact pads from corrosion due to any possible electrolyte leakage, ensuring more reliable and less resistive external connections to the sensors.

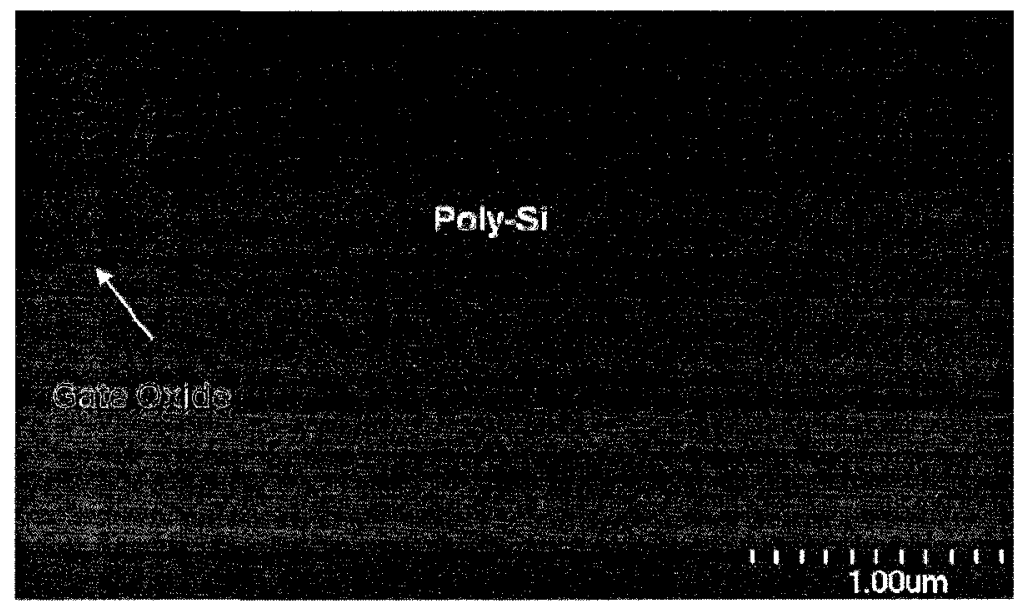

Figure 3.5 - SEM cross section of the gate and adjacent area after the wet etch of polysilicon.

Deposition of the chromium-gold layer was carried out by patterning, thermal evaporation, and lift off in N-methyl-2-pyrrolidone (NMP) at $80{ }^{\circ} \mathrm{C}$ for 5 minutes. These processes formed a layer of $100 \mathrm{~nm}$ of gold onto $20 \mathrm{~nm}$ of chromium on top of the poly-oxide or oxide gates. The common metal lift-off fabrication process involves annealing the chromium-gold coating at relatively low temperature and short time in 
order to make the metal surfaces uniform and smooth. However, it was decided to skip this annealing step since tests on similar samples showed chromium would diffuse through the thick gold layer and reach the surface. A blend of chromium and gold at the sensor surface would have reduced the density of available binding sites for immobilization of the thiolated DNA molecules and compromised the sensor sensitivity.

\subsubsection{Device Testing}

Following the fabrication and post-processing steps, it was required to test the devices to verify their properties before exposing them to the electrolyte. Therefore, each chip was probed to its external connection pads and I-V, C-V, and sub-threshold measurements were performed on each device. But first, the drain to substrate junction of each bioFET was checked with a simple diode test. These tests characterize the device as a whole, as well as the junctions, channel, and the gate. Any impurities or damages to the device would appear on the tests. For all the tests, the global substrate connection on the chip was shorted to the common source connection, which disabled the source to substrate $\mathrm{p}-\mathrm{n}$ junction diode.

The $\mathrm{p}$-type drain and the $\mathrm{n}$-type substrate form a $\mathrm{p}-\mathrm{n}$ junction diode and the main purpose of the drain to substrate diode test is to ensure this $\mathrm{p}-\mathrm{n}$ junction performs as a good diode. Not only does the diode test check the drain to substrate diode characteristics, but also it ensures the connectivity of the corresponding metal connections such as the wire-bonds, the contact pads, and also the aluminum lines. Figure 3.6 shows the drain to substrate diode characteristics of a few of the devices in the 
samples under test. As Figure 3.6 presents, all the devices show a very similar behaviour which verifies a proper diode characteristic, as well as good metal connections to the device.

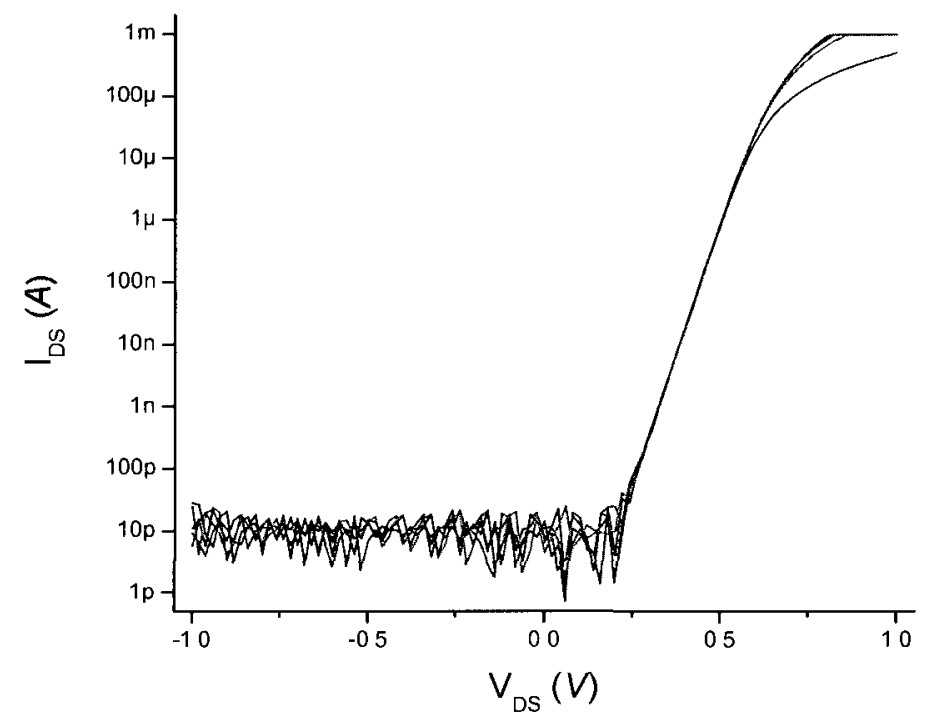

Figure 3.6 - Drain to Substrate diode characteristics of several bioFET sensors.

Next, $\mathrm{C}-\mathrm{V}$ measurements were taken at $1 \mathrm{MHz}$ in order to check the operation of the device's channel region. The drain voltage was set to a constant (low) voltage, and the gate voltage was swept with respect to the substrate. The source was also shorted to the substrate. Figure 3.7 presents a $\mathrm{C}-\mathrm{V}$ curve for a gold-chromium-poly-oxide gate, which does not demonstrate a hysteresis. As seen on the plot, applying a relatively large with respect to the threshold voltage - negative voltage on the gate with respect to the substrate enters the channel region into accumulation state. As the gate voltage is 
increased towards positive voltages, the channel enters into depletion and subsequently into inversion region of operation.

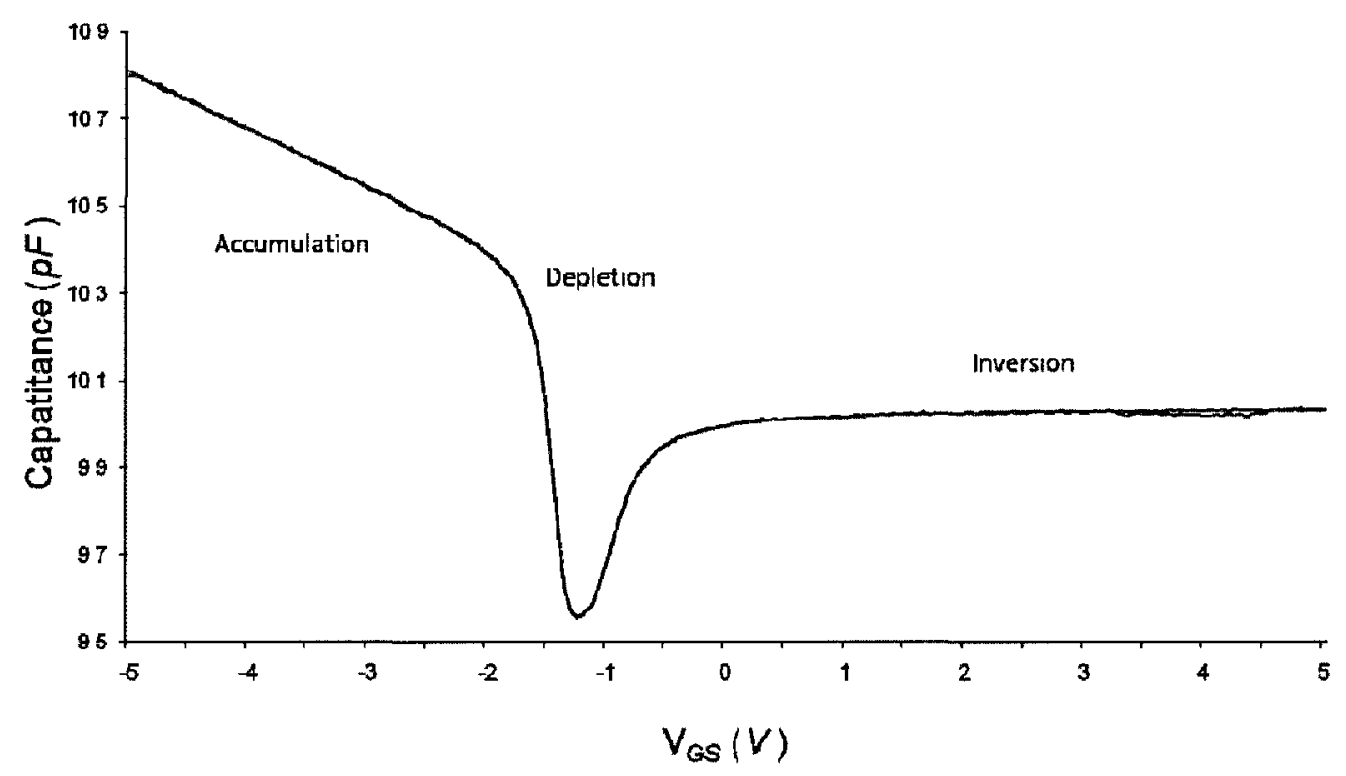

Figure 3.7 - C-V characteristics of a fully processed device with a gold-chromiumpoly-oxide gate. The channel region operation can be verified as it passes through accumulation, depletion, and inversion modes of operation.

Since in the $\mathrm{C}-\mathrm{V}$ experiments, the source was biased at the same voltage as the substrate and drain, (signal ground) varying the bias gate voltage also created the depletion area between the source and the device channel region, as well as the drain and channel. These depletion areas are dependent on the applied gate voltage and their capacitance add to the channel region's capacitance. This effect describes the non-flat linear portion of the $\mathrm{C}-\mathrm{V}$ curve at the accumulation region, as well as the high capacitance inversion region. 
The presence of the accumulation, depletion, and the inversion regions in the $\mathrm{C}-\mathrm{V}$ curves confirms the proper operation of the channel area. Similar behaviour was observed on devices with the removed poly layer gates.

After taking the $\mathrm{C}-\mathrm{V}$ measurements, each device was tested for its sub-threshold behaviour. As presented in Figure 3.8 the steep sub-threshold slopes indicate the devices could turn on and off very quickly, which is a good indication of low presence of impurities in the gate. The sub-threshold slope for the gold-chromium-poly-oxide gate and the gold-chromium-oxide gate were $84.3 \mathrm{mV} / \mathrm{dec}$ and $87.8 \mathrm{mV} / \mathrm{dec}$, respectively. However, the device with the poly layer in its gate has slightly smaller sub-threshold slope, and to some extent, larger sub-threshold leakage current. Although these differences are minor, they could be explained by the presence of the thick layer of poly, considerably increasing the gate's overall equivalent resistance.

Subsequent to the drain to substrate diode verifications, $\mathrm{C}-\mathrm{V}$ measurements, and subthreshold characterizations, each device was characterized by its I-V curve behaviour. The biofETs were treated as long channel devices. The I-V curves of each bioFET describe its operation as a FET, where the sub-threshold, linear, and saturation regions of operation can be determined. The device's threshold voltage is another property of the bioFET which was extracted from the corresponding I-V curves. Figure 3.9 demonstrates the plots of the I-V curves for two bioFETs with polysilicon layer and two bioFETs with the polysilicon layer removed. 


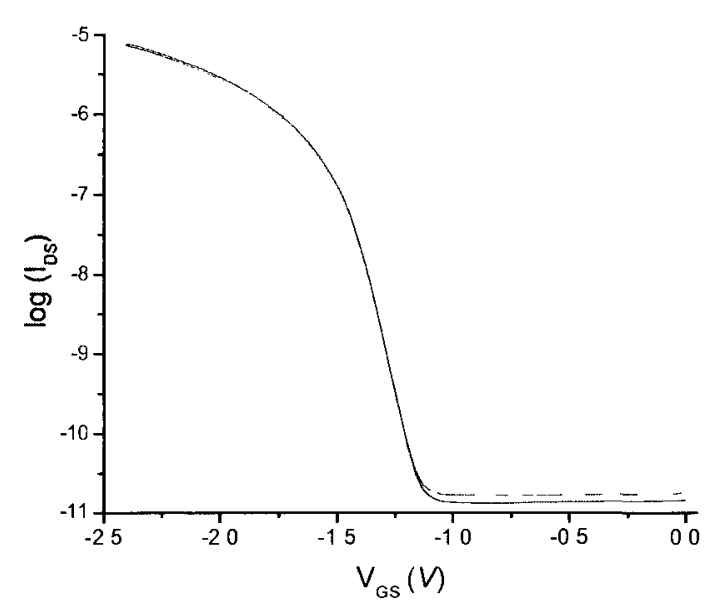

(a) Gold-chromium-poly-oxide gate

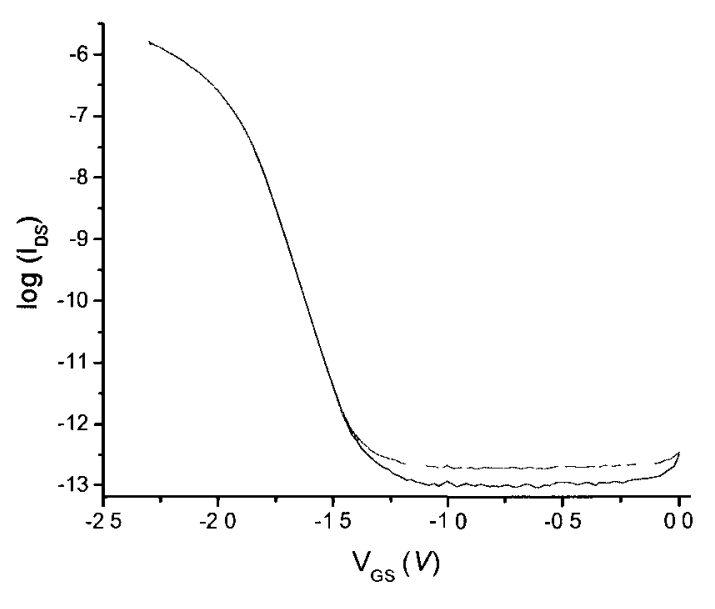

(b) Gold-chromium-oxide gate

Figure 3.8 - Sub-threshold plot of both types of the fully processed devices.

As seen on Figure 3.9, all devices showed similar behaviour: similar pinch-off voltage, a very flat and stable saturation current, similar knee voltage - drain to substrate voltage at which the curves switches from the linear region to the saturation region. While devices on a single chip showed similar characteristics, their threshold voltage varied. This dissimilarity could possibly be caused by the non-uniform nitride dry etch step during the post-processing, which caused non-identical gate exposure area. Therefore, variation in the opening window of the gate area could have resulted in divergence in the threshold voltages of different devices. This effect is illusrated in Figure 3.9 . 


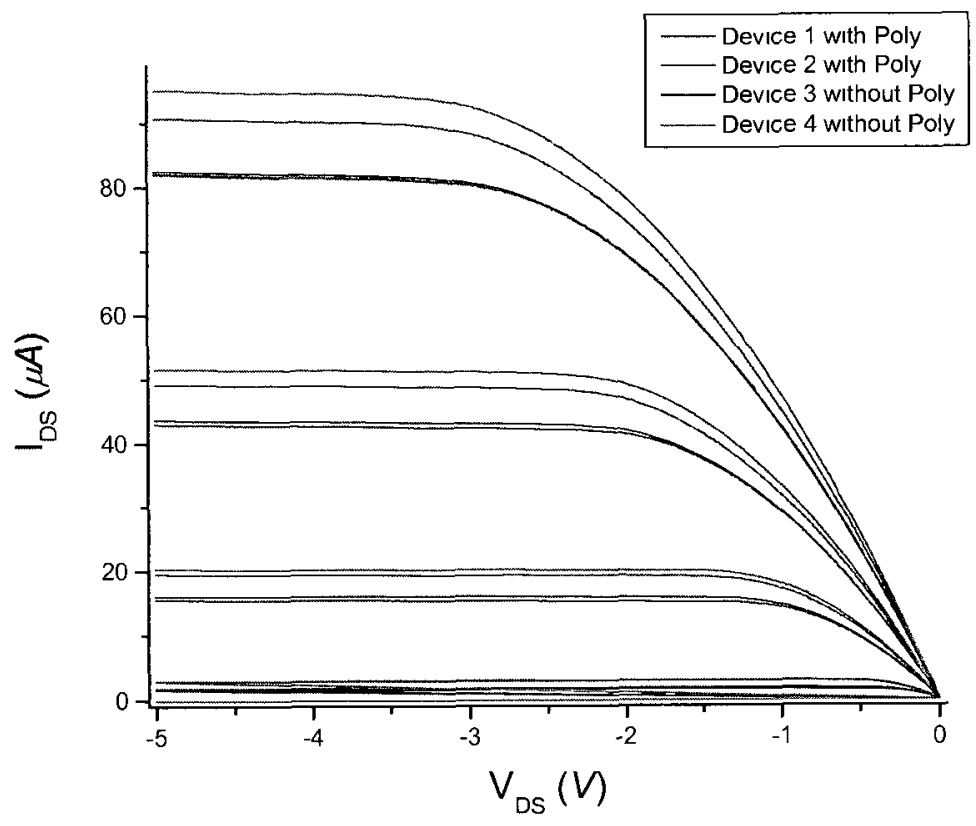

Figure 3.9 - I-V characteristic plots of various fully postprocessed devices with and without poly layer on their gates.

Not only did the above tests characterize the devices, but they also checked the devices for any impurities or damage (especially at the gate), which could have influenced the detection sensitivity during the final measurements.

\subsubsection{Packaging}

Following the tests characterizing the devices on each chip, the chips were packaged for electrolyte encapsulation and external connection during the final measurements. Each chip was mounted onto a standard 84-pin header using conductive silver epoxy, and subsequently wire-bonded to the header with gold wires. The choice of gold for the wires was due to its stable bonding onto the gold coated contact pads, and its 
immunity to corrosion due to any possible electrolyte leakage. A $3 \mathrm{~mm}$ high, $1 \mathrm{~cm}$ wide, and $1 \mathrm{~mm}$ thick glass ring was glued to the chip surface using non-conducting epoxy. The ring was utilized to enclose the electrolyte only at the region where the devices under test were exposed, and also to prevent a short circuit between the electrolyte and the external contact pads or wire-bonds. In order to ensure the wire bonds were intact during the measurements, they were covered by non-conductive epoxy. The packaged chip was subsequently ready for measurements in the electrolyte. Figure 3.10 shows a top view of the finished chip.

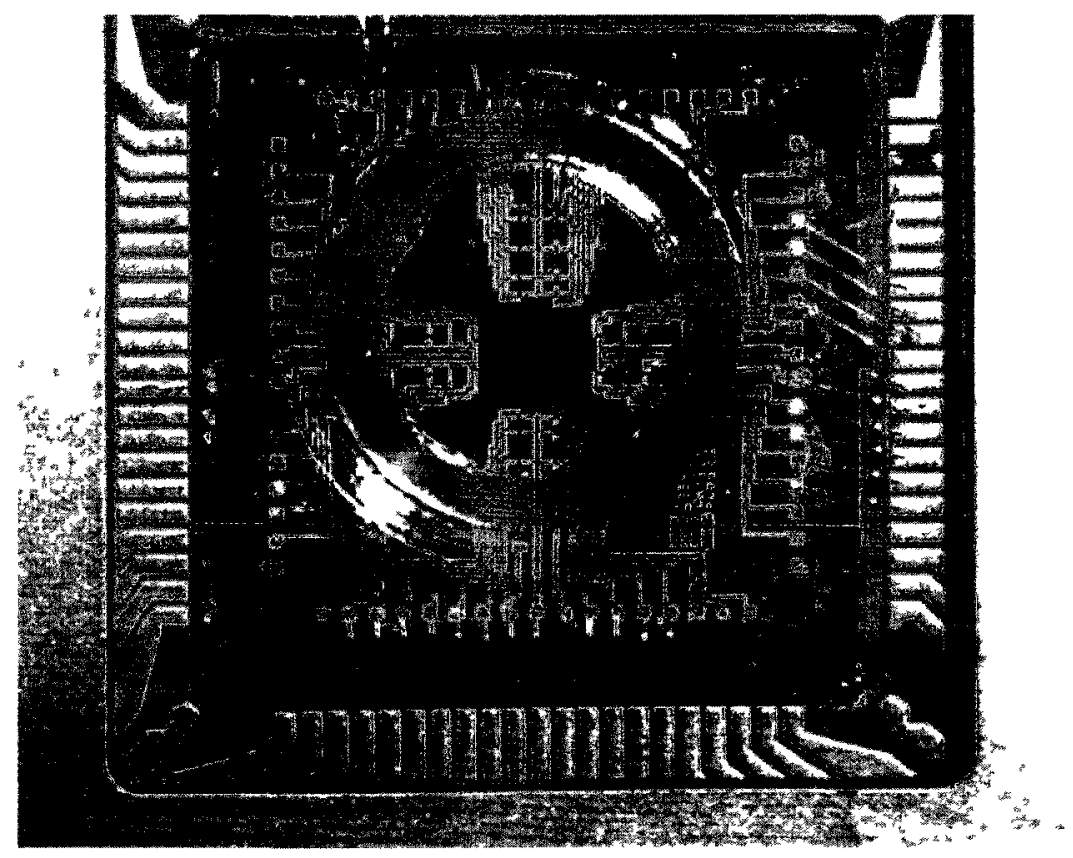

Figure 3.10 - Top view of the fabricated and post-processed bioChip. The devices under test are visible in the middle rows. All the devices to be measured are exposed to the electrolyte solution inside the glass ring. 


\section{Chapter 4}

\section{Measurement Setup and Testing}

A bio-sensing measurement involves detection of the presence of biological molecules in an electrolyte at the surface of the bioFET sensor and conversion into a measurable electrical signal. This chapter will discuss the system setup to do the measurements and the measurement procedure.

\subsection{Measurement Setup}

The chip containing the sensors is the heart of the DNA detection system using bioFETs. The bioFET measurements involved measuring the sensor's responses to changes in the ionic strength of the electrolyte. Such responses were observed at three steps; with no DNA molecule attached to the bare gold surface, after attaching the ssDNA to the bioFET surface, and after hybridizing the dsDNA molecule. The ionic strength measurements were used to detect the presence of DNA molecules at the bioFET sensor surface.

The change in the ionic strength of the electrolyte is due to the presence or absence of the DNA molecules. This change modifies the charge distribution at the bioFET-electrolyte interface, resulting in a change in the transistor current which could be translated into a shift in the threshold voltage of the bioFET. With the purpose of the bioFET experiments, the system shown in Figure 4.1 was made. It includes electrical 
connections to the chip via the socket, the reference electrode, and the electrolyte handling system.

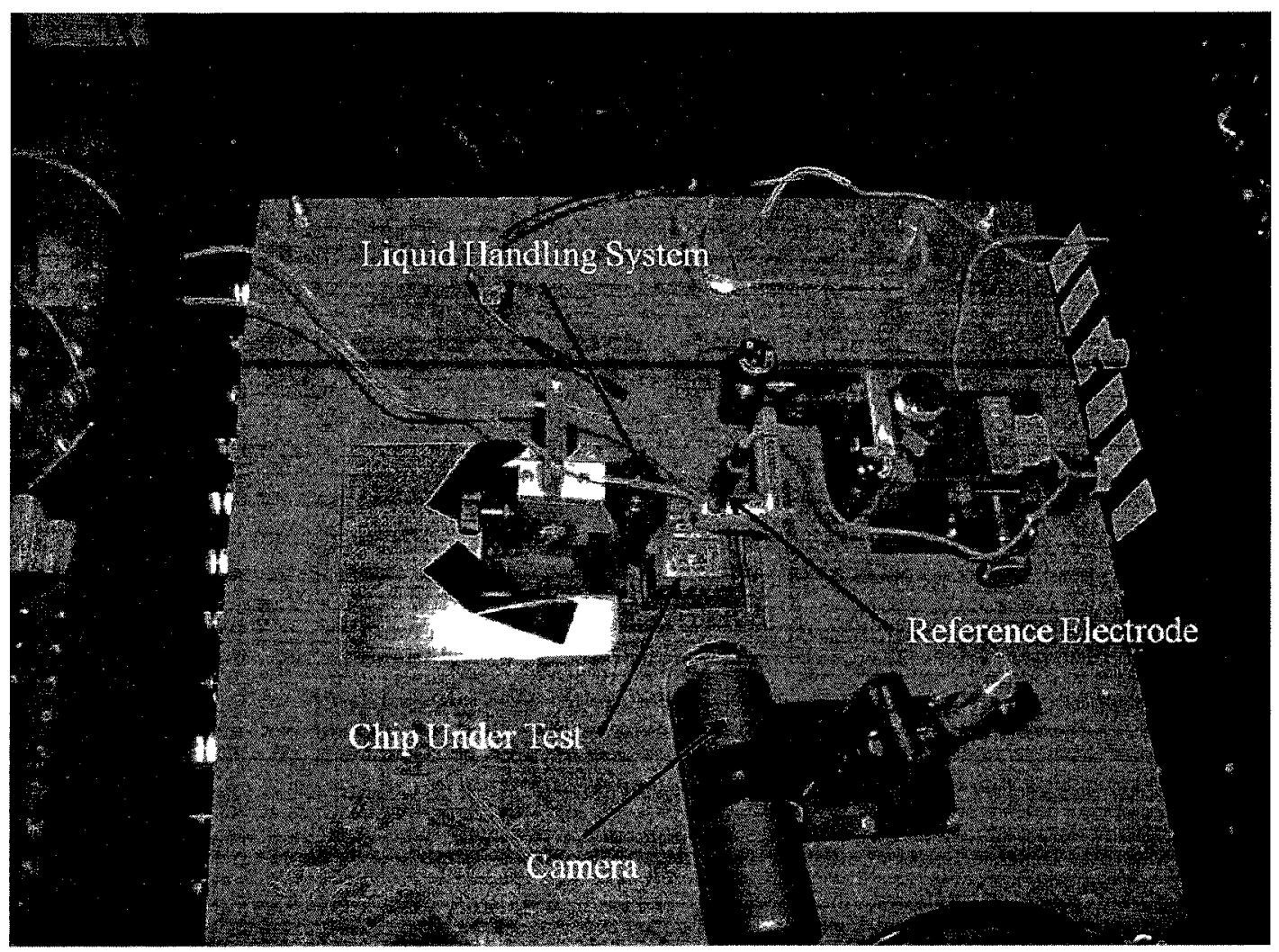

Figure 4.1 - Overview of the experimental setup.

\subsubsection{Electrical Readings}

The electrical measurements of the bioFET sensors consisted of characterizing the bioFET devices through $\mathrm{I}-\mathrm{V}$ and sub-threshold curves, and real-time drift measurements in the electrolyte. Figure 4.2 shows the schematic view of the measurement system. In this setup, the common source and global substrate terminal connections of the transistors were connected to the common signal ground, in order to remove the FETs' back-gate 
effect, commonly known as the body effect. All other voltages were referenced with respect to this signal ground.

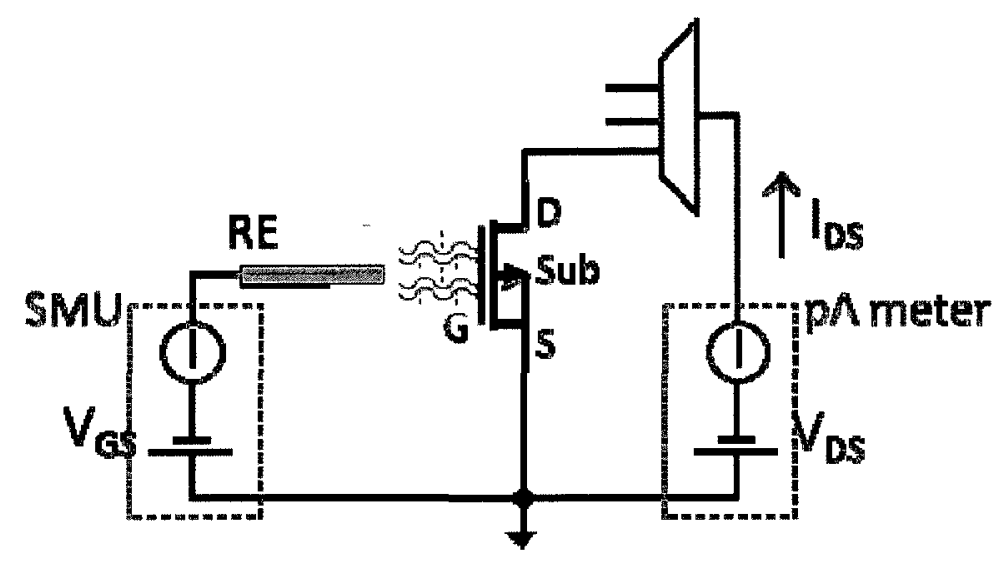

Figure 4.2 - Schematic of the measurement system.

The electrolyte was biased with the reference electrode at a constant voltage, $\mathrm{V}_{\mathrm{GS}}$. A Keithley 236 source measurement unit (SMU) supplied $V_{G S}$, and constantly measured the gate to substrate leakage current, $\mathrm{I}_{\mathrm{GS}}$. The drain voltage, $\mathrm{V}_{\mathrm{DS}}$, was provided with an HP 4140 Pico ammeter, which also continuously read the drain to source current, IDs. In order to switch between difference devices in the array, an HP 5250 switching matrix was employed. The switching matrix was programmed to sequence between the devices in a way that only one FET is turned on and measured at a time. The turn on time of each device was very short and only a small number of devices were measured periodically; hence, once the device is turned on, its $I_{D S}$ level would go back to the same level as before it was turned off. 
A piece of software was written to control the SMU, pA meter, the switching matrix, and the electrolyte flow. The software supplied the appropriate voltage on the device drains and the reference electrode, recorded their currents, and handled the switching between the devices. It also provided the options for sub-threshold characteristics, I-V measurements, and time drift readings.

In order to remove any possible variation of the bias from one reading to the next, the SMU, pA meter, the switching matrix, and also all of their connections shared the same signal ground and a common device ground. This allowed biasing $V_{G S}$ and $V_{D S}$ with respect to a common ground voltage. Hence, triaxial cables were used for interconnects among the reading apparatus, as well as the measurement site. An interconnecting triaxial cable constituted the centre signal, common signal ground on the inner shield, and device earth ground on the guard. Shielding the signal and signal ground provided a high immunity to the ambient electrostatic and electromagnetic interferences for the centre signal.

Additionally, the entire measurement site was enclosed inside a stainless steel box connected to the earth ground. The box shared the same common earth ground as the reading apparatus' device ground, and it shielded the chip, reference electrode, and the electrolyte against external electromagnetic and electrostatic interferences. Additionally, the box was painted in black to avoid any reflected light exposed to the reference electrode, devices and the electrolyte. 


\subsubsection{Threshold Voltage Shift}

The bioFET's sensitivity to the presence of a DNA molecule or electrolyte shift is dependent on the charge distribution changes occurring at the device and electrolyte interface. The change in the charge distribution itself is quantifiable by finding the threshold voltage shift of the bioFET caused by any changes at the device surface. During the fabrication and post-processing stages, all possible preventative measures were taken into the account to ensure the interface surface state density of the bioFETs remain low during the experiments. The threshold voltage shift is calculable from the change in the drain to source current. At steady states, $I_{D S}$ is most stable over time at the saturation region of the bioFET's operation. As mentioned in section 2.3.3, in this region of operation, the relationship between $\mathrm{I}_{\mathrm{DS}}$ and $\mathrm{V}_{\mathrm{T}}$ at any drain voltages above the saturation voltage is as Equation 4.1 shows.

$$
I_{D S}=\mu_{n} C_{o x} \frac{W}{L} \frac{\left(V_{G S}-V_{T}\right)^{2}}{\alpha 2}
$$

From Equation 4.1, it can be found that:

$$
\sqrt{I_{D S}}=\beta\left(V_{G S}-V_{T}\right)
$$

where:

$$
\beta=\sqrt{\frac{\mu_{n} C_{o x}}{\alpha 2} \frac{W}{L}}
$$


Hence, a change on the effective threshold voltage of a sensing device at a constant reference electrode voltage, $\mathrm{V}_{\mathrm{GS}}$, would reflect on its drain current. In other words:

$$
\Delta \sqrt{I_{D S}}=-\beta \Delta V_{T}
$$

Equations 4.3 and 4.4 confirm that in the steady state, the relationship between the square root of the drain current and the effective threshold voltage is only dependent on the device characteristics determined at the design, fabrication and post-processing. Therefore, a shift in the threshold voltage is only the consequence of a change in the bioFET surface charges. However, it should be noted that in Equations 4.2 to 4.4 the effect of temperature has been assumed to be negligible. Although the measurements were performed in room temperature, if the devices heated up due to switching, Equation 4.2 should be modified to take the temperature changes into effect. Since the switching was relatively slow, and it did not cause a significant increase in the temperature, for the simplicity of the experiments the temperature effects were assumed insignificant, and thus ignored.

In other words, if one plots the square root of the drain current versus the gate voltage, any change in the threshold voltage would appear as a shift in the curve. This concept is shown in Figure 4.3 for one of the bioFETs under the test, where both the curves are similar, but one of the curve's threshold voltage is shifted by $60.0 \mathrm{mV}$ from the other. 


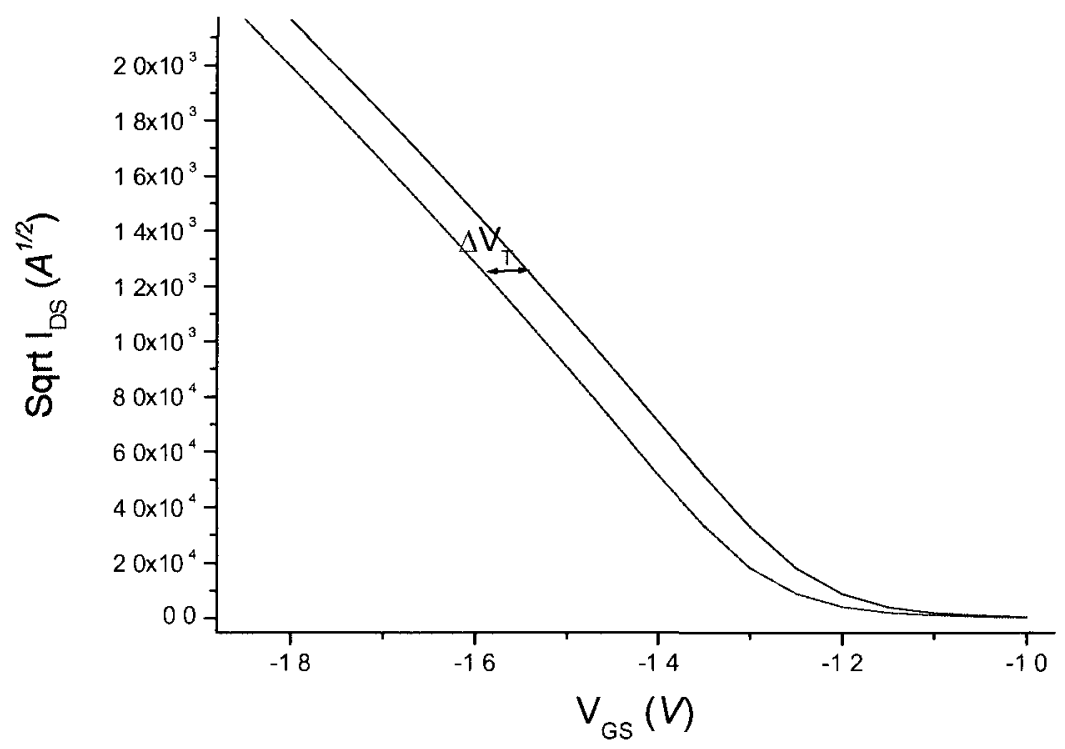

Figure 4.3 - Threshold voltage shift extraction from the shift in $\sqrt{I_{D S}}$ vs. $\mathrm{V}_{\mathrm{GS}}$ curve for a fully processed bioFET device.

Nevertheless, as Figure 4.3 illustrates, the $I_{D S}-V_{G S}$ curves are divided into two segments. The first section is the non-linear region below the threshold voltage which does not follow Equation 4.2. This behaviour is due to the irregular sub-threshold behaviour of the non-ideal devices, which was not a focus of interest in this project. The second section of the curve is the linear region above the threshold voltage, where Equation 4.2 is exclusively valid. All the calculations and measurements for this project were performed based on this region of the $I_{D S}-V_{G S}$ curve, ensuring Equation 4.2 was completely applicable.

For the purpose of this project, the bioFETs were first characterized by taking their I-V plots at each circumstance. Then the relationship between the square root of $I_{D S}$ 
and $V_{G S}$, at a certain $V_{D S}$ above saturation was considered. From of the $\sqrt{I_{D S}}-V_{G S}$ plot, the threshold voltage of the device - under this specific condition - was calculated and compared to the ones from other circumstances.

Subsequently, a fixed gate voltage common to all devices was chosen from the linear region of the $\sqrt{I_{D S}}-V_{G S}$ curves, well above the threshold voltage. Next, a realtime source to drain current drift measurement was performed using the selected gate voltage and an appropriate constant $V_{D S}$ above $V_{D S}$ sat. The drift measurements provided real-time visualization of $\mathrm{I}_{\mathrm{DS}}$, which were next converted into meaningful threshold voltage versus time drift plots, using Equation 4.4. As discussed in section 4.1.4, it should be noted that the work function of the reference electrode was assumed to be invariable and did not contribute to the threshold voltage shift. The same applies for the electrolyte.

\subsubsection{Electrolyte}

The electrolyte in the ring had to be a buffer with stable ionic strength to reflect the steady bias voltage of the reference electrode onto the gate. The two electrolytes used in the experiments were $5 \mathrm{mM}$ phosphate buffer saline (PBS) solutions. The solutions were regulated at $\mathrm{pH} 7.23$, where DNA molecules are most stable. This is the natural $\mathrm{pH}$ of the living cells, and also near the blood's, $\mathrm{pH} 7.4$. Additionally, the saturated buffer inside the reference electrode's capillary was also secured at slightly basic solutions, such as pH7.23. Although the solutions were prepared just before the measurements, they were monitored regularly for any $\mathrm{pH}$ changes. Throughout the measurements their $\mathrm{pH}$ did not change from 7.23 . 
The two types of PBS solutions were buffered with potassium chloride salt with ionic strengths of $0.3 \mathrm{M}(2 \mathrm{X})$ and $0.015 \mathrm{M}(0.1 \mathrm{X})$. Potassium chloride was chosen as the buffer salt, since it is widely used in stable chemical solutions, its ionization reactions are well reproducible, and it provides highly electropositive potassium ions and extremely electronegative chloride ions. Tests on a few other highly electropositive ions in the electrolyte showed potassium ions, unlike sodium ions, do not have a significant impact on the interface surface state density of the FETs, and they are more electropositive than calcium ions. Additionally, a high concentration of chloride ions in the electrolyte reduces the chance of ions leaking from the $\mathrm{Ag}-\mathrm{AgCl}$ reference electrode. This improves the reference electrode's stability in maintaining a constant bias voltage.

With the purpose of keeping a high density of potassium and chloride ions available in the electrolyte inside the glass ring well, fresh solution was continuously pumped into the well at a rate of $40 \mathrm{~mL} / \mathrm{hr}$ with one syringe pump per each buffered solution. Switching between the buffers was feasible by switching between the pumps. The excess liquid in the ring was also pushed out using another syringe pump at a rate of $52 \mathrm{~mL} / \mathrm{hr}$, faster than the inlet in order to reduce the chance of liquid overflow from top of the open ring.

Flow of the solution from the syringe reservoirs to the ring, and from the ring to the waste syringe creates an ionisable path that could introduce electrostatic interferences into the system. To minimize such interference, polytetrafluoroethylene (PTFE) commercially known as the brand name Teflon - tubes were used for connecting the ring to the syringe pumps. PTFE is the superior preference given that it is extremely 
hydrophobic, does not produce electrostatic charges through the buffer flow, and it is very flexible. Moreover, the tubes were cut to the shortest optimal length such that first, liquid would reach the ring sooner; and secondly the effective resistance of the PBS solution inside the tubes would decrease. A smaller resistance - in case of an electrostatic interference at the solution - ensures that the voltage difference between the point of stimulation and the measurement site, the sensor surface, would be at its smallest possible value. Ultimately, glass needles provided electrostatic immune junctions between the tube lines. The entire liquid flow system was enclosed inside an electrically grounded aluminum box, guarded against ambient electromagnetic interferences. The above precautions reduced any risk associated with the introduction of any electrostatic and electromagnetic interference into the solution that could influence the sensor performance.

\subsubsection{Reference Electrode}

A reproducible and stable bioFET measurement requires charges at the sensorelectrolyte interface to be in the steady state. Therefore, the voltage at the electrolyte should also be stable. Given that the reference electrode sets this voltage, it needs to sustain a steady potential throughout the entire measurement. For the course of the experiments in this project, a commercial reference electrode, Dri-Ref-450 by World Precision Instruments, was used. The Dri-Ref-450 reference electrode is a $450 \mu \mathrm{m}$ thick $\mathrm{Ag}-\mathrm{AgCl}$ wire enclosed in a glass capillary filled with almost-saturated $3 \mathrm{M}$ potassium chloride buffered solution. This electrode was an excellent choice since the buffer exchange in the membrane at the tip of the electrode is negligible $\left(\sim 5 \times 10^{-10} \mathrm{~mL} / \mathrm{hr}\right)$. 
The theoretical work function of the Dri-Ref-450 reference electrode changes only at a rate of $0.1 \mathrm{mV} /$ minute in steady state, providing a stable potential. However, over a period of time, the work function changes due to the shift in the behaviour of the oxidation-reduction reactions in the electrode. Therefore, the stability behaviour was required to be verified over the course of the experiments. For this purpose, the reference electrode's half cell potentials, with respect to a standard saturated calomel electrode, were looked at in different solutions. Diagrams in Figure 4.4 show the half cell potential of the reference electrode in $2 \mathrm{X}$ and $0.1 \mathrm{X}$ buffers stabilizing over 30 minutes. Each curve on the plots shows the test at the beginning of each measurement day. Also, Figure 4.5 compares the reference electrode stabilization behaviour in the same day.

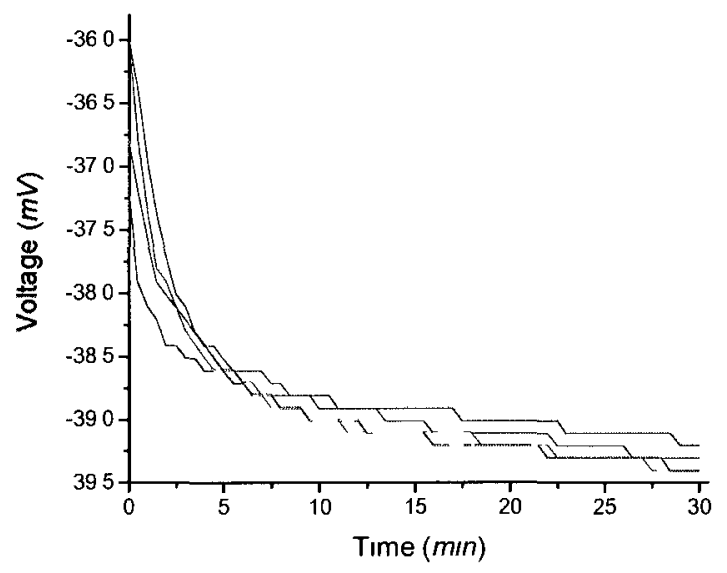

(a) $2 \mathrm{X} \mathrm{KCl}$ buffered solution

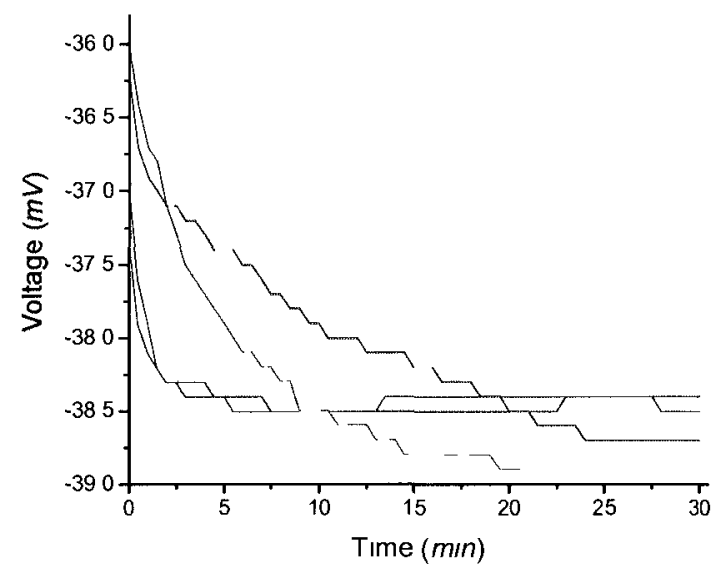

(b) $0.1 \mathrm{X} \mathrm{KCl}$ buffered solution

Figure 4.4 - Reference electrode stabilizing over time with respect to a standard electrode.

Each curve on the plots shows the test at the beginning of each measurement day.

As the plots of Figure 4.4 illustrate, the reference electrode's galvanized charges were just about stabilized within the 30-minutes test. Additionally, the stabilized 
reference electrode showed similar behaviours in various tests over time. Although theoretically the work function should have trend of possible drift over time, it did not follow a recognizable trend. This is shown in Figure 4.4. Additionally, it was assumed that the electrode's work function would not change significantly throughout the sameday experiments. Moreover, the plots of Figure 4.4 and Figure 4.5 show that the difference between the work functions of the two types of buffers is approximately 0.5 $\mathrm{mV}$.

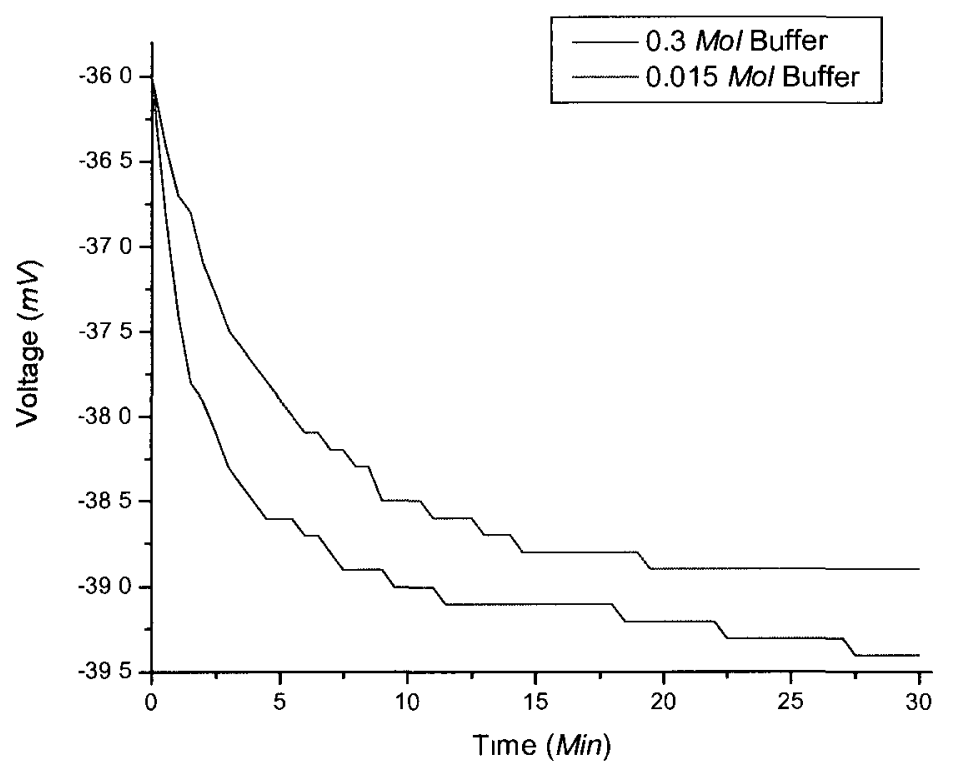

Figure 4.5 - Comparison of the reference electrode stabilization behaviour (with respect to a standard electrode) in two solutions with different ionic strengths measured on the same date. 


\subsection{Experimental Procedure}

The bioFET measurements began with verifying the sensitivity of the sensors to the solutions without any DNA molecules at the sensor's gold coated surface. These measurements ensure the stability of the devices in the buffered solution, and also characterize the transistors' behaviour in contact with the electrolyte.

\subsubsection{Bare Gold Surface}

The sensing site of each bioFET is its electron-rich gold-coated gate surface. Although the gold layer does not react with the electrolyte, once it is exposed to a buffered aqueous solution, it "lends" parts of its free electrons to the numerous -OH groups floating in the solution. This results in forming a monolayer of $-\mathrm{OH}$ groups at the sensor-electrolyte interface to create binding sites, and facilitating the attachment of the biological molecules to the sensor surface. Any presence of contaminations caused by any ambient biological species at the surface of the sensor could partially passivated the -

$\mathrm{OH}$ groups, and compromise the bioFET measurements. Even though preventive measures were taken into account to ensure no contamination would reach the sensors' surface, there was a necessity to clean the surface of the chip. Therefore, the packaged chip under the test was treated with atomic oxygen plasma for 10 minutes. This cleaning step ensured the number of the $-\mathrm{OH}$ groups present at the sensor surface would be at its maximum level [184].

After the device verification and cleaning steps, the first stage of the measurements in the bioFET project was measuring the response of each device to the 
electrolyte in different ionic strengths while no biological molecules were attached at the surface. This ensured the stability of the device in the electrolyte and also made a base point for comparison in the subsequent functionalization and hybridization steps. The $\mathrm{I}-\mathrm{V}$ characterization and real-time drift measurements provided preliminary device characteristics based on the threshold voltage shift.

\subsubsection{Functionalization}

In order to attach a DNA strand to the gold surface, one should functionalize it. The functionalization process in this project involved immobilizing a ssDNA molecule to the gold surface utilizing a thiol linker. The thiol group acted as a receptor for the DNA molecule and allowed targeting a specific oligonucleotide sequence [174]. The thiolterminated DNA molecules were prepared at the Department of Chemistry at Carleton University. For Functionalization, first the solution inside the glass well was poured out, and then $40 \mu \mathrm{L}$ of the buffered solution containing the thiol-terminated DNA molecules was dispensed into the well. The well was covered with a glass slide and allowed to sit for 120 minutes at room temperature. During this period, the thiol group passivated the surface $-\mathrm{OH}$ groups and resulted in binding the sulfhydryl $\left(-\mathrm{C}-\mathrm{S}^{-}\right)$end of the thiol group to the gold surface. This is shown in Figure 4.6. After the completion of the DNA functionalization, the chip was rinsed using the DNA buffer in order to ensure the nonfunctionalized DNA molecules were safely removed. 

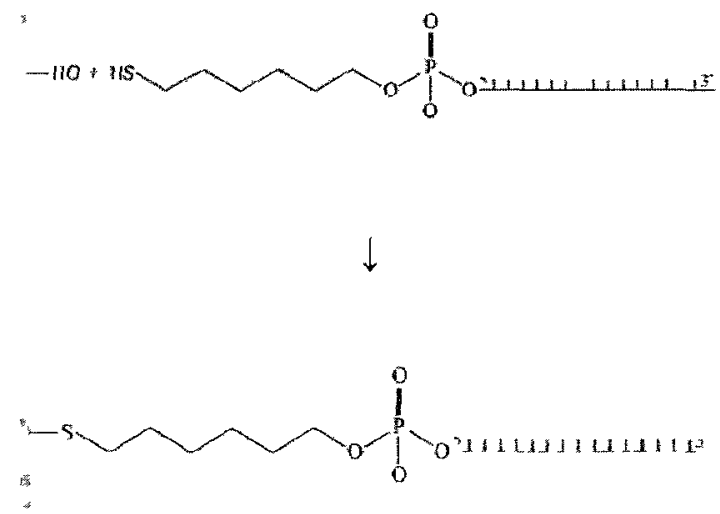

Figure 4.6 - An illustration of the bioFET functionalization through thiol linkers, passivating the $-\mathrm{OH}$ groups at the sensor surface

Following the bioFET functionalization process, the I-V characterization and the real-time drift measurements in both buffered solutions were performed on the devices. The difference in the threshold voltage shift obtained from this stage to the one from the first step supported estimating the concentration of the functionalized DNA molecules. As discussed in section 2.5, a large increase in the output signal in response to the DNA functionalization process is expected.

\subsubsection{Hybridization}

Subsequently, the glass ring was emptied once more, and $40 \mu L$ of the buffered solution containing the complementary DNA strand was introduced to the well. The solution was allowed to sit for another 120 minutes in room temperature to hybridize the already immobilized DNA molecules, as shown in Figure 4.7. In general, possibility of the non-complementary DNA sequences immobilizing to the unused binding sites of the sensor surface is extremely vital to the hybridization measurement. 
In any experiment, if the unused binding sites are passivated by such immobilization, the bioFET threshold voltage variation due to the second immobilization would significantly affect the sensitivity of the sensor. However, the complementary DNA strands lacked the thiol linkers, and also the binding of the DNA sequence to the gold surface was very unstable over a period of time. As a result, it was assumed that a very small and insignificant number of the unused binding sites were passivated by the complementary DNA molecules, and these sites did not contribute to the shift in the threshold voltage change due to the hybridization process. An additional 5-minute rinsing step ensured the unhybridized DNA strands floating in the electrolyte have been removed and would not compromise the measurements subsequent to hybridization.

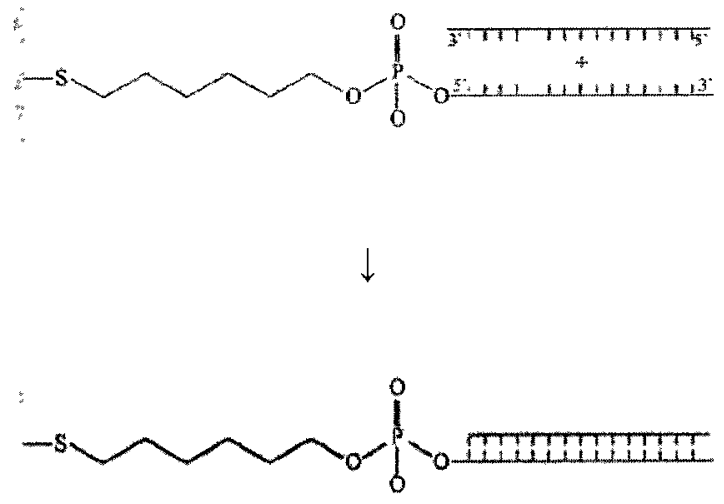

Figure 4.7 - An illustration of the bioFET hybridization at the surface of the sensor

A final $\mathrm{I}-\mathrm{V}$ characterization and real-time drift measurements in both buffered solutions lead to the extraction of the threshold voltage shift due to the hybridization of the DNA sequence. The threshold voltage shift due to the functionalization allowed approximating the density of the hybridized DNA molecules. Knowing the efficiency of 
the functionalization and hybridization processes, if this threshold voltage shift is compared with those of the bare gold surface and functionalization steps, one would be able to extract the sensitivity of the bioFET sensor. As discussed in section 2.5, an increase in the output signal in response to the DNA hybridization process is expected. However, this change is expected to be smaller than the change in response to the functionalization process.

It should be noted that in order to verify the efficiency of the functionalization and hybridization processes, sample test chips received treatments under identical conditions. These chips were then measured using the X-ray photoelectron spectroscopy (XPS). From the XPS results it was learned:

- The gold spectra proved that there was not any gold oxide layers present at the sensor interface, denoting that the entire gold surface was available for immobilizing DNA molecules.

- The 2-p phosphorus and $1-\mathrm{s}$ nitrogen spectra indicated that the DNA functionalization and hybridization steps on the surface of all samples had been successful. The height of the peaks suggested that approximately an equal amount of DNA was present on all samples.

- The gold and silicon spectra suggested that even if the deposited gold layer was lower than expected, DNA hybridization and attachment would still be successful. 
The XPS measurements were not performed in an organic-free environment. Therefore, due to a very high risk of contaminations during the XPS measurements, the sample chips could not be analyzed by electrical measurements after the XPS measurements. 


\section{Chapter 5}

\section{Results and Discussions}

\subsection{Results}

As discussed in sections 2.5, and 4.2, the measurement process involved measuring the device current drift due to the change in electrolyte concentration at $2 \mathrm{X}$ and $0.1 \mathrm{X}$, before the DNA functionalization and again before and after hybridization. From the current drifts, the threshold voltage shifts in response to the changes in the electrolyte in each stage were calculated and compared. This section will discuss the measurements, and analysis of such shifts.

\subsubsection{Gold-Chromium-Oxide Gate Devices}

While 12 devices in the chip were fabricated, post-processed and exposed to the electrolyte, 3 of them were not accessible, due to the physical damage to their external contact pads. Of the 9 available devices, unfortunately 5 of them either did not behave like a FET (possibly due to defected gates or channels) or did not show any response to the electrolyte (possibly due to damaged sensor surface). Therefore, the measurements were performed on 4 devices simultaneously.

First, all four devices were characterised by taking their $\mathrm{I}-\mathrm{V}$ curves in both $0.1 \mathrm{X}$ and $2 \mathrm{X}$ buffered solutions. From the $\mathrm{I}-\mathrm{V}$ plots, the corresponding $\sqrt{I_{D S}}$ vs. $V_{G S}$ curves 
were plotted. The slopes of the $\sqrt{I_{D S}}$ vs. $V_{G S}$ curves at their linear region, regions with $V_{G S}$ well above the turn-on voltage, gave their corresponding $\beta$ value described in Equation 4.2. The results, presented in Table 5.1, show that, in general the all four devices have similar $\beta$ values. Moreover, each device has very comparable $\beta$ values in both solutions. This is expected since $\beta$ is dependent on the physical characteristics of the devices set in the design, fabrication and post-processing steps.

\begin{tabular}{|l|c|c|c|c|}
\hline & Device 1 & Device 2 & Device 3 & Device 4 \\
\hline$\beta_{01 \times}\left(\times 10^{-3} \frac{\sqrt{A}}{v}\right)$ & -3.7 & -3.36 & -3.79 & -3.87 \\
\hline$\beta_{2 \times}\left(\times 10^{-3} \frac{\sqrt{A}}{v}\right)$ & -3.55 & -3.34 & -3.80 & -3.83 \\
\hline
\end{tabular}

Table 5.1 - Calculated values of $\beta$ for each device in $0.1 \mathrm{X}$ and $2 \mathrm{X}$ buffered solutions on bare gold surfaces.

Next, simultaneous real-time measurements of the output current of each device, $\mathrm{I}_{\mathrm{DS}}$, with respect to time were performed. $\mathrm{V}_{\mathrm{GS}}$ and $\mathrm{V}_{\mathrm{DS}}$ were biased at $-1.6 \mathrm{~V}$ and $-0.8 \mathrm{~V}$, respectively. The measurements started with $2 \mathrm{X}$ buffered solution, because of more stability at this concentration. Once the behaviours of the devices were stable, the electrolyte was switched to $0.1 \mathrm{X}$; and when stable again, the liquid feed was switched back to the $2 \mathrm{X}$ electrolyte. There was approximately a 20 second gap from the point that the buffer was switched until the response was seen on the sensors. This can be explained by the fact that the new buffer needed about 20 seconds to replace the entire volume of the old buffer solution in the glass ring. The electrolyte switching cycle was repeated few times in order to ensure reproducibility of the output. The response of each device to the 
ionic strength changes in the electrolyte varied; however, in general, most of the devices showed analogous behaviours. The output is shown in Figure 5.1.a).

The change in the electrolyte concentration created transitions which took a while to settle. The devices had a tendency to drift further. Although different in each device, each drift was determined to have a constant rate over time. This means even though the transition had completed, the system and also the reference electrodes were still responding to the change. Baseline subtraction was performed and such drifts were taken into consideration when extracting the signal shift, characterized by the threshold voltage shift. Figure 5.1 shows the output current $\mathrm{I}_{\mathrm{DS}}$ measured by the sensor.

By using the $\beta$ values of each device, and according to Equation 4.4, the output current drifts were transformed into plots of the threshold voltage offset vs. time drifts. The term offset means the calculated values are not the actual values for the threshold voltages. They are only offsets from a reference value. Since the calculated threshold voltage offset in one solution is only valid for the same solution, two drift plots per device were plotted. The difference between the values of the threshold voltage plot of the $2 \mathrm{X}$ electrolyte (at stable region) and that of $0.1 \mathrm{X}$ represent the threshold voltage shift in response to the change in the concentration of the electrolyte. This is shown in Figure 5.1.b). The valid results for each device were averaged and Table 5.2 shows a summary of the shifts in the threshold voltage in response to the electrolyte concentration transitions. 


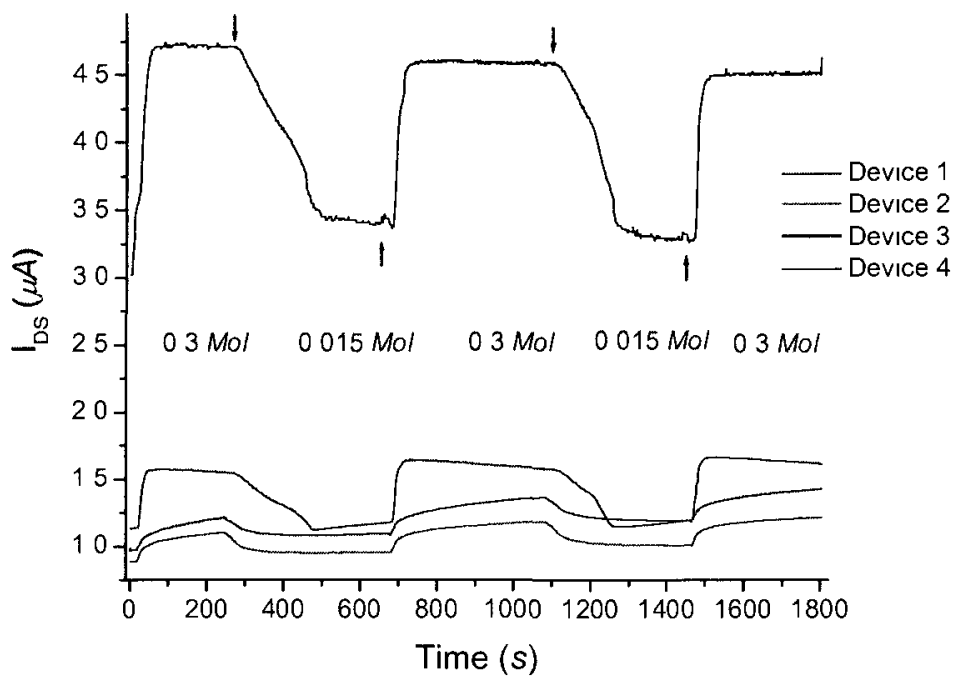

(a)

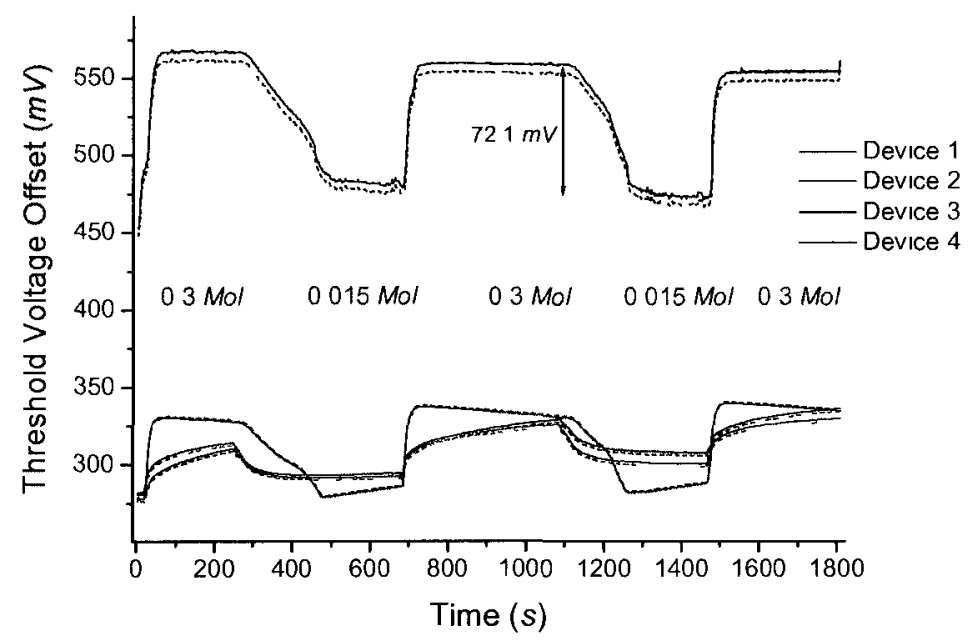

(b)

Figure 5.1 - (a) Measurement of the output current, $\mathrm{I}_{\mathrm{DS}}$, over time before functionalization for all 4 devices. The points where the transitions were made can be seen by the wine and olive pointers on the plot for the $2 \mathrm{X}$ to $0.1 \mathrm{X}$ switch, and $0.1 \mathrm{X}$ to $2 \mathrm{X}$ switch, respectively; (b) Threshold voltage change vs. time before functionalization. The Solid lines show the calculated threshold voltage changes based on the behaviour of the devices in $2 \mathrm{X}$ solution, while the dashed lines are based on their behaviour in $0.1 \mathrm{X}$ solution. 


\begin{tabular}{|c|c|c|c|c|}
\hline & Device 1 & Device 2 & Device 3 & Device 4 \\
\hline$\Delta \mathrm{V}_{\mathrm{T} 01 \mathrm{X} \text { to } 2 \mathrm{X}(\mathrm{mV})}$ & 34.9 & 36.2 & 44.2 & 70.0 \\
\hline$\Delta \mathrm{V}_{\mathrm{T} 2 \mathrm{X} \text { to } 01 \mathrm{X}(\mathrm{mV})}$ & 26.8 & 23.2 & 41.4 & 68.8 \\
\hline
\end{tabular}

Table 5.2 - Summary of threshold voltage shifts before functionalization.

In spite of the drifts, Table 5.2 and Figure 5.1.b) show that all four devices have similar behaviours. Devices 1 and 2, and to less extent, device 3 show signal drifts. However, in general, the bare gold surfaces of all four devices show high sensitivity to the changes in electrolyte concentration prior to the DNA functionalization. The change was more noticeable in device 4 .

Subsequently, the entire chip was treated with the thiol-terminated ssDNA solution to functionalize the devices. After the functionalization process was complete, all four devices were characterized again in both buffered solutions in order to obtain their $\beta$ from their corresponding $I-V$ curves. Table 5.3 shows the resultant $\beta$ values which again are very similar and close to the ones obtained from before the functionalization process. The best performance is seen in device 4 , whose $\beta$ values are identical.

\begin{tabular}{|l|c|c|c|c|}
\hline & Device 1 & Device 2 & Device 3 & Device 4 \\
\hline$\beta_{01 \times}\left(\times 10^{-3} \frac{\sqrt{\mathrm{A}}}{\mathrm{v}}\right)$ & -3.61 & -3.62 & -3.76 & -3.83 \\
\hline$\beta_{2 \times}\left(\times 10^{-3} \frac{\sqrt{\mathrm{A}}}{\mathrm{V}}\right)$ & -3.41 & -3.55 & -3.56 & -3.83 \\
\hline
\end{tabular}

Table 5.3 - Calculated values of $\beta$ for each device in $0.1 \mathrm{X}$ and $2 \mathrm{X}$ buffered solutions after functionalization. 
The real-time measurements provided the current vs. time plots of Figure 5.2.b). In order to sustain the measurement conditions, $V_{G S}$ and $V_{D S}$ were biased at the same voltage as prior to functionalization, at $-1.6 \mathrm{~V}$ and $-0.8 \mathrm{~V}$, respectively. These plots were transformed into the threshold voltage offset $v$ s. time plots of Figure 5.2.b) using the values indicated in Table 5.3. Device 2 showed to have a noticeably larger threshold voltage compared to the other three bioFETs. Therefore, operating in the same voltage as the other three devices resulted in this device to be in the non-linear region of $\sqrt{I_{D S}}$ vs $V_{G S}$ curves. On the other hand, increasing the drain voltage would result in very large currents of device 3, and particularly device 4, which could compromise the device operation. Hence, it was decided to operate devices 2 and 1 (whose behaviour is closer to device 2) separately from devices 3 and 4 . Therefore, devices 3 and 4 were operated at $\mathrm{V}_{\mathrm{GS}}$ of $-1.2 \mathrm{~V}$.

As the summary illustrated in Table 5.4 shows, immobilization of the thiolterminated ssDNA molecules to the sensors surfaces produced a larger increase in the threshold voltage shifts due to the changes in the ionic strength of the electrolyte. The increase in the threshold voltage shifts is an indication of the presence of the negatively charged ssDNA molecules at the bioFETs' surface, resulting in a larger charge density at the channel-insulator interface. This change is relatively smaller than the change in response to the transition of the electrolyte concentration. However, it is large enough to demonstrate the presence of the DNA molecule. 


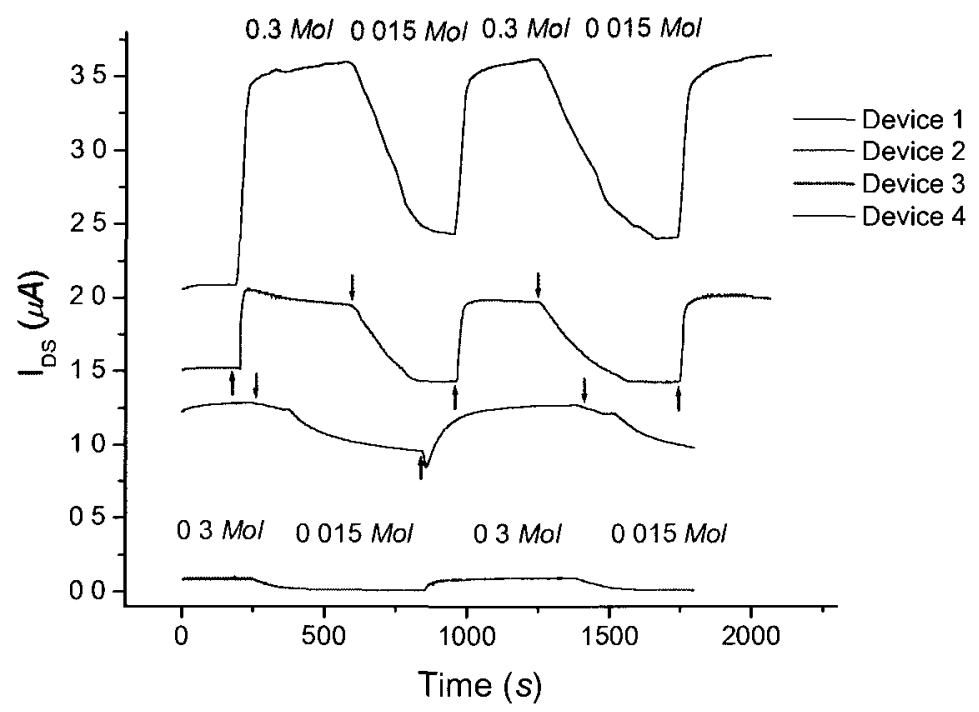

(a)

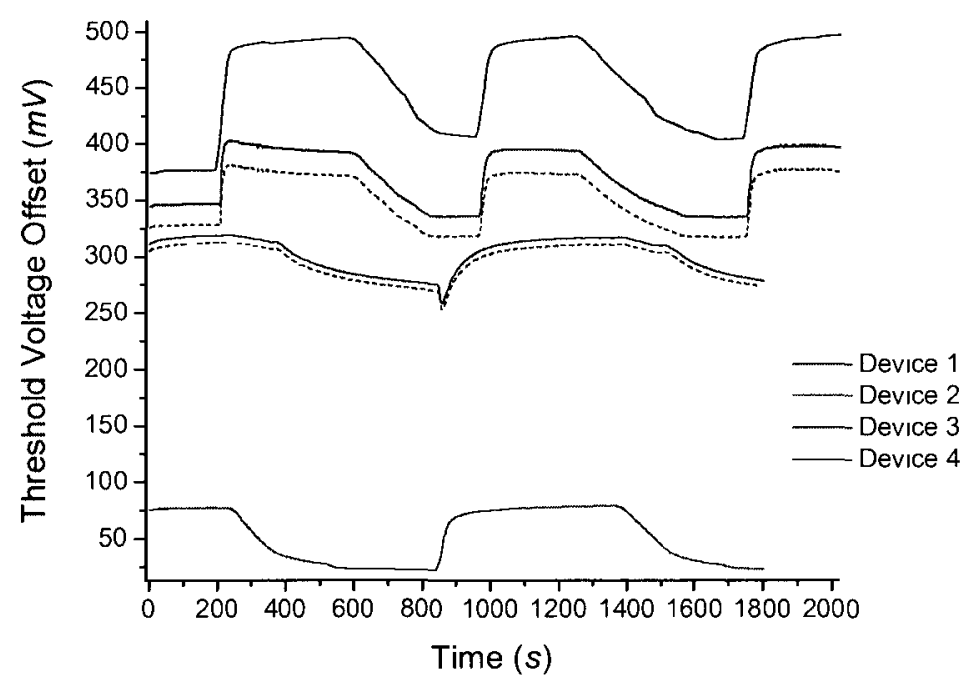

(b)

Figure 5.2 - (a) Output current, IDs vs. time after functionalization;

(b) Threshold voltage vs. time after functionalization. 


\begin{tabular}{|l|c|c|c|c|}
\hline & Device 1 & Device 2 & Device 3 & Device 4 \\
\hline$\Delta \mathrm{V}_{\mathrm{T} 0 \text { IX to 2X }(\mathrm{mV})}$ & 43.1 & 47.2 & 75.2 & 89.7 \\
\hline$\Delta \mathrm{V}_{\mathrm{T} 2 \mathrm{X} \text { to 0 1X }(\mathrm{mV})}$ & 34.2 & 43.7 & 76.0 & 91.2 \\
\hline $\begin{array}{l}\text { Change in } \Delta \mathrm{V}_{\mathrm{T} 0 \text { 1X to 2X }} \\
\text { due to functionalization (mV) }\end{array}$ & 8.2 & 11.0 & 31.0 & 19.7 \\
\hline $\begin{array}{l}\text { Change in } \Delta \mathrm{V}_{\mathrm{T} 2 \mathrm{X} \text { to 0 1X }} \\
\text { due to functionalization (mV) }\end{array}$ & 7.4 & 20.5 & 34.6 & 22.4 \\
\hline
\end{tabular}

Table 5.4 - Summary of threshold voltage shifts after functionalization.

At last, the devices were hybridized, and the bioFETs were again characterized for their $\beta$ values, presented in Table 5.5. Once again, similar $\beta$ values for all four devices, and also consistency from previous steps confirmed all four devices have very similar physical characteristics in their channels. The real-time current vs. time measurements are illustrated in Figure 5.3.a), and are followed by the produced threshold voltage offset drift plots of Figure 5.3.b). For the same reason explained above, devices 1 and 2 were measured separately from devices 3 and 4 . Therefore, while $V_{D S}$ were set -0.8 $\mathrm{V}, \mathrm{V}_{\mathrm{DS}}$ was biased at $-1.6 \mathrm{~V}$ and $-1.2 \mathrm{~V}$ for measurements of devices 1 and 2 , and devices 3 and 4 , respectively.

\begin{tabular}{|l|c|c|c|c|}
\hline & Device 1 & Device 2 & Device 3 & Device 4 \\
\hline$\beta_{01 \times}\left(\times 10^{-3} \frac{\sqrt{A}}{V}\right)$ & -3.55 & -3.61 & -3.72 & -3.78 \\
\hline$\beta_{2 \times}\left(\times 10^{-3} \frac{\sqrt{A}}{v}\right)$ & -3.53 & -3.50 & -3.66 & 3.78 \\
\hline
\end{tabular}

Table 5.5 - Calculated values of $\beta$ for each device in $0.1 \mathrm{X}$ and $2 \mathrm{X}$ buffered solutions after hybridization. 


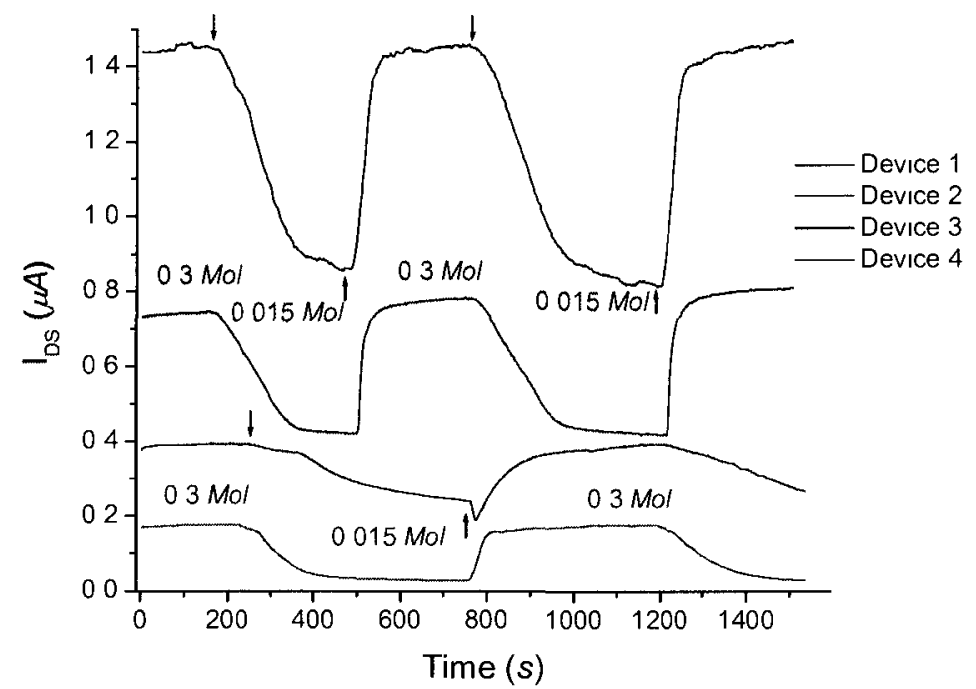

(a)

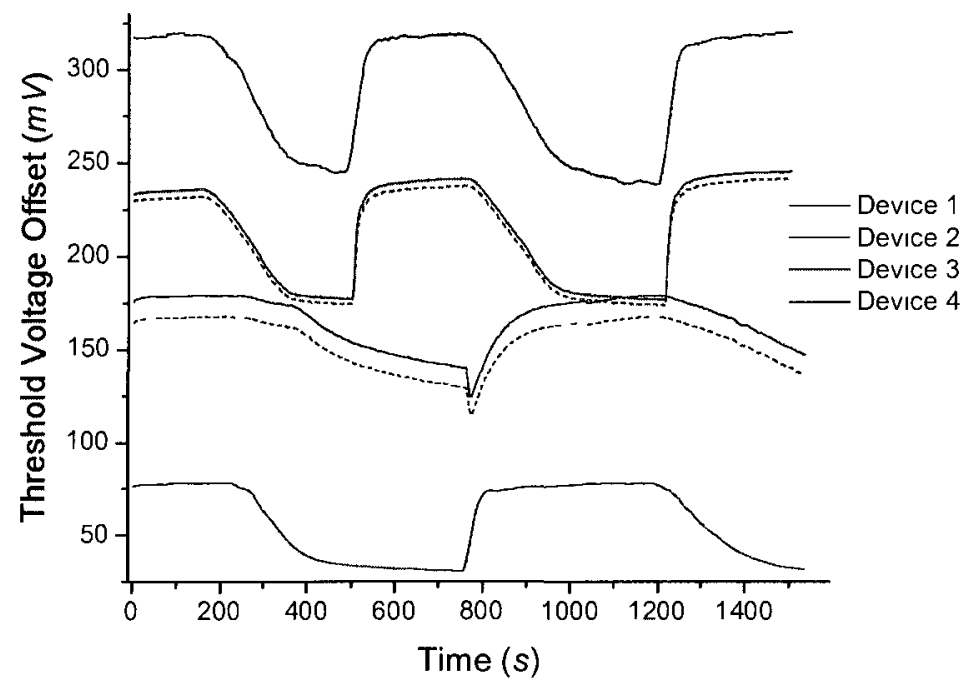

(b)

Figure 5.3 - (a) Output current, I Ds vs. time after hybridization;

(b) Threshold voltage $v s$. time after hybridization. 
The summary of results presented in Table 5.6 shows that all four devices showed additional changes in the threshold voltage shifts after hybridization with the complementary strand. These changes are also believed to be caused by the DNA hybridization process. However, device 2, as discussed earlier shows a very small change and this could be explained by the possibility that it enters the non-linear region of the $\sqrt{I_{D S}}$ vs. $V_{G S}$ curve when operating in contact with the $0.1 \mathrm{X}$ electrolyte.

\begin{tabular}{|l|c|c|c|c|}
\hline & Device 1 & Device 2 & Device 3 & Device 4 \\
\hline$\Delta \mathrm{V}_{\mathrm{T} \text { 0 1 } 1 \text { to 2X }(\mathrm{mV})}$ & 19.2 & 48.1 & 67.5 & 73.5 \\
\hline$\Delta \mathrm{V}_{\mathrm{T} 2 \mathrm{X} \text { 10 0 1X }}(\mathrm{mV})$ & 27.8 & 42.2 & 67.7 & 79.6 \\
\hline $\begin{array}{l}\text { Change in } \Delta \mathrm{V}_{\mathrm{T} 01 \mathrm{X} \text { to } 2 \mathrm{X}} \\
\text { due to hybridization (mV) }\end{array}$ & -23.9 & 0.9 & -7.7 & -16.2 \\
\hline $\begin{array}{l}\text { Change in } \Delta \mathrm{V}_{\mathrm{T} 2 \mathrm{X} \text { to 0 1X }} \\
\text { due to hybridization (mV) }\end{array}$ & -6.4 & -1.5 & -8.3 & -11.6 \\
\hline
\end{tabular}

Table 5.6 - Summary of threshold voltage shifts after hybridization.

As it was discussed in section 4.1.4, the $\mathrm{Ag}-\mathrm{AgCl}$ reference electrode used for these measurements behaved differently in the two electrolytes, and had a work function difference of $0.5 \mathrm{mV}$ between the two ionic strengths. The measurement results should be compensated by this work function offset. However, the results presented above are significantly larger than the offset, and this work function offset is negligible in the final results. 


\subsubsection{Gold-Chromium-Poly-Oxide Gate Devices}

10 out of the chip's 12 devices exposed to the electrolyte were accessible through their contact pads. And another 5 devices either did not behave like a FET or had no sensitivity to the electrolyte for the same reasons explained in section 5.1.1, leaving the simultaneous wet measurements with 5 devices. The same process described in section 5.1.1 was performed on these devices.

First, $\beta$ values of all five devices in both electrolyte concentrations, as presented in Table 5.7, are very similar, and in a very close range. Different from the devices without the poly layer because of their overall gate's characteristics, the comparable $\beta$ values confirms the physical characteristics of all five devices are very similar. This means they have been treated similarly during the fabrication process.

\begin{tabular}{|l|c|c|c|c|c|}
\hline & Device 1 & Device 2 & Device 3 & Device 4 & Device 5 \\
\hline$\beta_{01 \times}\left(\times 10^{-3} \frac{\sqrt{A}}{\mathrm{~V}}\right)$ & 2.59 & -2.57 & -2.58 & -260 & -2.61 \\
\hline$\beta_{2 \times}\left(\times 10^{-3} \frac{\sqrt{\mathrm{A}}}{\mathrm{V}}\right)$ & -2.53 & -2.51 & -2.63 & -2.61 & -2.58 \\
\hline
\end{tabular}

Table 5.7 - Calculated values of $\beta$ for each device in $0.1 \mathrm{X}$ and $2 \mathrm{X}$ buffered solutions before functionalization.

Measuring the device current vs. time in the bare gold surfaces, as Figure 5.4.a) shows, all five devices have small drifts but with constant rates over time. These drifts are believed to be results of the drift in the reference electrode. The results analysis for these devices compensated for the drifts, considering the constant rates do not affect the 
threshold voltage shift in the transitions. The optimal $V_{G S}$ and $V_{D S}$ biases were $-1.85 \mathrm{~V}$ and $-0.7 \mathrm{~V}$, respectively.

The analysis of the threshold voltage shifts shows all five devices were very sensitive to the transition in the electrolyte concentration, as they have very large threshold voltage shifts. The discrepancy in the results shown in Table 5.8 between the calculated values based on $\beta$ values of $2 X$ and $0.1 X$ electrolytes are most likely because of the drifts over time. Even though they were taken into account, the drifts compromised the results. However, since the $2 \mathrm{X}$ to $0.1 \mathrm{X}$ transition stabilized much quicker, results due to this transition are believed to be valid. Nevertheless, device 1 did not stabilize in the period the measurements were performed. This might be the reason it shows dissimilar behaviours.

\begin{tabular}{|c|c|c|c|c|c|}
\hline & Device 1 & Device 2 & Device 3 & Device 4 & Device 5 \\
\hline$\Delta \mathrm{V}_{\mathrm{T} 0.1 \mathrm{X} \text { to } 2 \mathrm{X}(\mathrm{mV})}$ & 27.1 & 55.6 & 73.2 & 69.0 & 83.4 \\
\hline$\Delta \mathrm{V}_{\mathrm{T} 2 \mathrm{X} \text { to } 01 \mathrm{X}(\mathrm{mV})}$ & 102.8 & 37.1 & 63.4 & 32.2 & 35.2 \\
\hline
\end{tabular}

Table 5.8 - Summary of threshold voltage shifts before functionalization. 


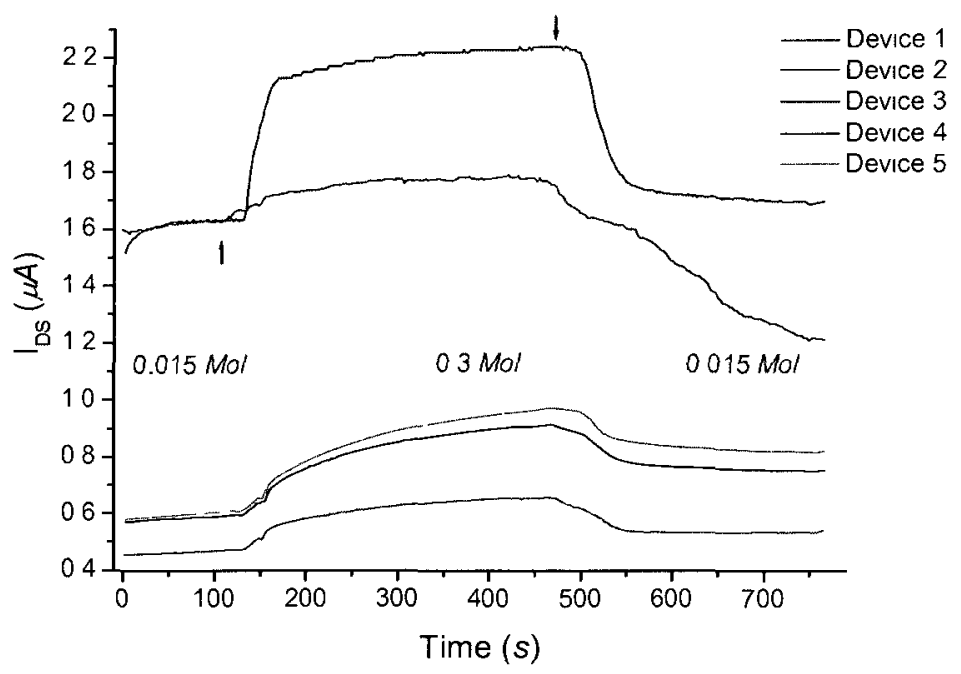

(a)

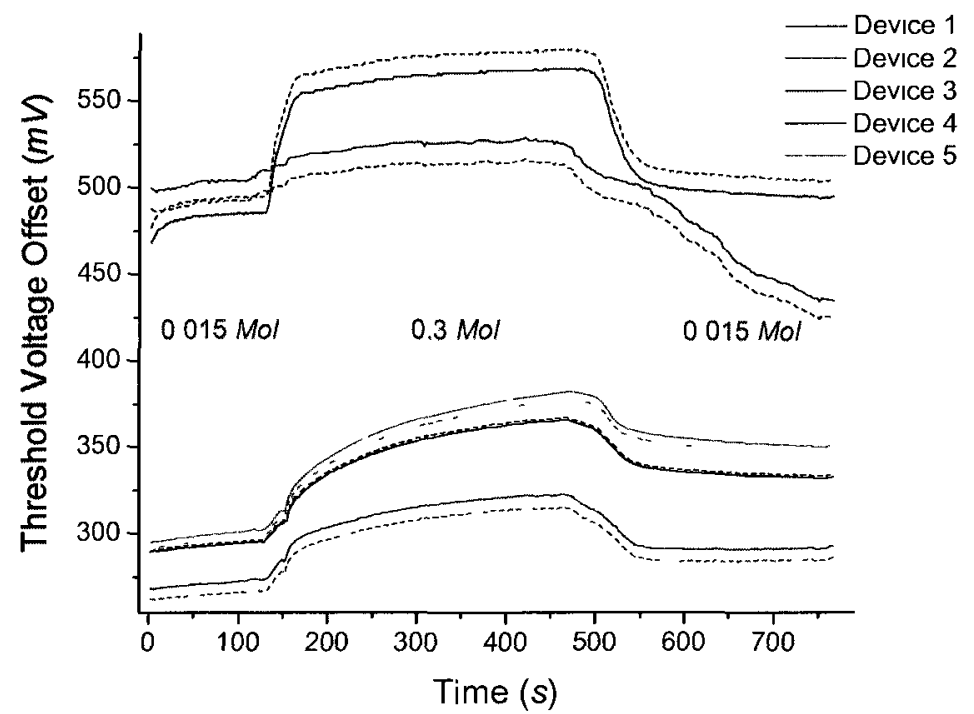

(b)

Figure $5.4-$ (a) Output current, IDs $v s$. time before functionalization;

(b) Threshold voltage vs. time before functionalization. 
Subsequently, all the devices were functionalized with the ssDNA molecules, and characterized. As shown in Table 5.9, consistency of the $\beta$ values confirms there have not been any physical damages to the gate and channel regions of the devices. Presented in Figure 5.5.a), the signal drifts after the functionalization of the thiol-terminated ssDNA molecules to the sensors were still present. However, since they were smaller, they did not compromise the results. Reproducibility of the transitions verifies the drifts were insignificant on the overall performance of the five devices.

On the other hand, transitions from both low concentration to high concentration and vice versa, as shown in Figure 5.5.b) and Table 5.10 are very close. Yet again, device 2 operated with a lower output current due to its smaller threshold voltage. However, it operated in the saturated region. Again, in order to sustain the measurement conditions, $\mathrm{V}_{\mathrm{GS}}$ and $\mathrm{V}_{\mathrm{DS}}$ were biased at the same voltages as before functionalization, $-1.85 \mathrm{~V}$ and $0.7 \mathrm{~V}$, respectively.

\begin{tabular}{|l|c|c|c|c|c|}
\hline & Device 1 & Device 2 & Device 3 & Device 4 & Device 5 \\
\hline$\beta_{01 \times}\left(\times 10^{-3} \frac{\sqrt{\mathrm{A}}}{\mathrm{V}}\right)$ & -2.56 & -2.59 & -2.55 & -2.61 & -2.60 \\
\hline$\beta_{2 \times}\left(\times 10^{-3} \frac{\sqrt{\mathrm{A}}}{\mathrm{V}}\right)$ & -2.44 & -2.63 & -2.56 & -2.63 & -2.62 \\
\hline
\end{tabular}

Table 5.9 - Calculated values of $\beta$ for each device in $0.1 \mathrm{X}$ and $2 \mathrm{X}$ buffered solutions after functionalization. 


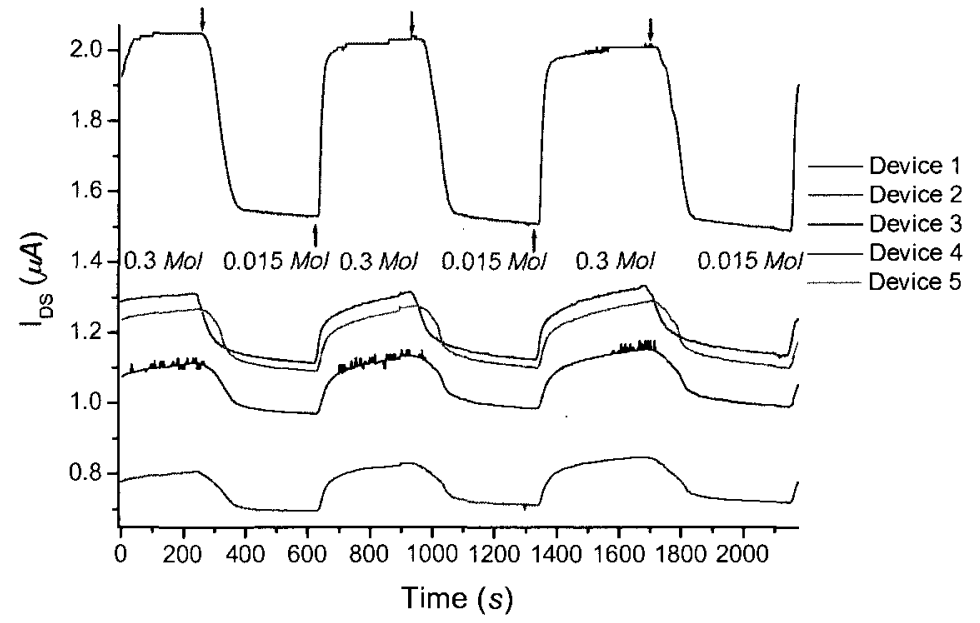

(a)

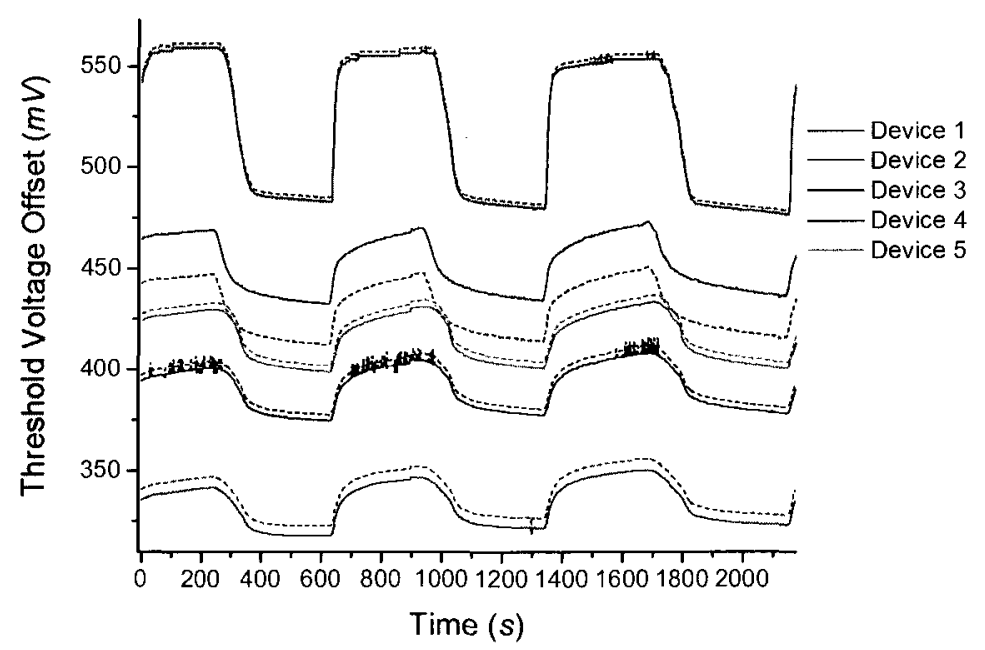

(b)

Figure 5.5 - (a) Output current, IDS $v s$. time after functionalization;

(b) Threshold voltage $v s$. time after functionalization. 


\begin{tabular}{|l|c|c|c|c|c|}
\hline & Device 1 & Device 2 & Device 3 & Device 4 & Device 5 \\
\hline$\Delta \mathrm{V}_{\mathrm{T} 0.1 \mathrm{X} \text { to 2X }(\mathrm{mV})}$ & 56.4 & 23.7 & 72.0 & 26.5 & 28.8 \\
\hline$\Delta \mathrm{V}_{\mathrm{T} 2 \mathrm{X} \text { to 0.1 } \mathrm{X}(\mathrm{mV})}$ & 54.8 & 21.7 & 74.6 & 23.7 & 28.8 \\
\hline $\begin{array}{l}\text { Change in } \Delta \mathrm{V}_{\mathrm{T} 0.1 \mathrm{X} \text { to 2X }} \\
\text { due to functionalization }(\mathrm{mV})\end{array}$ & 29.27 & -31.9 & -1.2 & -42.5 & -54.6 \\
\hline $\begin{array}{l}\text { Change in } \Delta \mathrm{V}_{\mathrm{T} 2 \mathrm{X} \text { to 0 1X }} \\
\text { due to functionalization }(\mathrm{mV})\end{array}$ & -48.0 & -31.9 & 11.2 & -8.5 & -6.4 \\
\hline
\end{tabular}

Table 5.10 - Summary of threshold voltage shifts after functionalization.

Since the results due to the low to high concentration transitions were assumed unacceptable because of the large drift, only the changes in the threshold voltage shifts due to functionalization in the high to low concentration transitions were considered. Although most devices seemed to show smaller change in the threshold voltage shifts after the functionalization, device 3 showed a larger change. This could be because its surface had been post processed slightly different than other, particularly during the nitride dry etch process.

Following these measurements, the DNA hybridization process was performed, and then the devices were characterized again. The $\beta$ values, presented in Table 5.11, were consistent and very close to each other. This was followed by the current vs. time measurements, at the same $V_{G S}$ and $V_{D S}$ biases as both before and after functionalization, $-1.85 \mathrm{~V}$ and $-0.7 \mathrm{~V}$, respectively. 


\begin{tabular}{|l|c|c|c|c|c|}
\hline & Device 1 & Device 2 & Device & Device 4 & Device 5 \\
\hline$\beta_{01 \times}\left(\times 10^{-3} \frac{\sqrt{\mathrm{A}}}{\mathrm{v}}\right)$ & -2.61 & -2.59 & -2.60 & -2.64 & -2.62 \\
\hline$\beta_{2 \times}\left(\times 10^{-3} \frac{\sqrt{\mathrm{A}}}{\mathrm{v}}\right)$ & -2.49 & -2.60 & -2.63 & -2.63 & -2.63 \\
\hline
\end{tabular}

Table 5.11 - Calculated values of $\beta$ for each device in $0.1 \mathrm{X}$ and $2 \mathrm{X}$ buffered solutions after hybridization.

The signal drifts with constant rates appeared again for most devices after hybridizing the devices with the complementary DNA strands in plots of in Figure 5.6. Once again, since the $\beta$ values did not change significantly from the previous steps, the drifts were mostly likely caused by the solution or the reference electrode. The changes in the threshold voltage shifts, caused by the hybridization and shown in Table 5.12, are different among the five devices. The hybridization caused small changes in the threshold voltage shift for most devices, except device 5. While devices 2 and 4 showed large signals, devices 1,3 , and 5 showed smaller ones. The difference is presumably due to the lack of uniformity in the sensor surface which causes discrepancy during the functionalization and hybridization processes. 


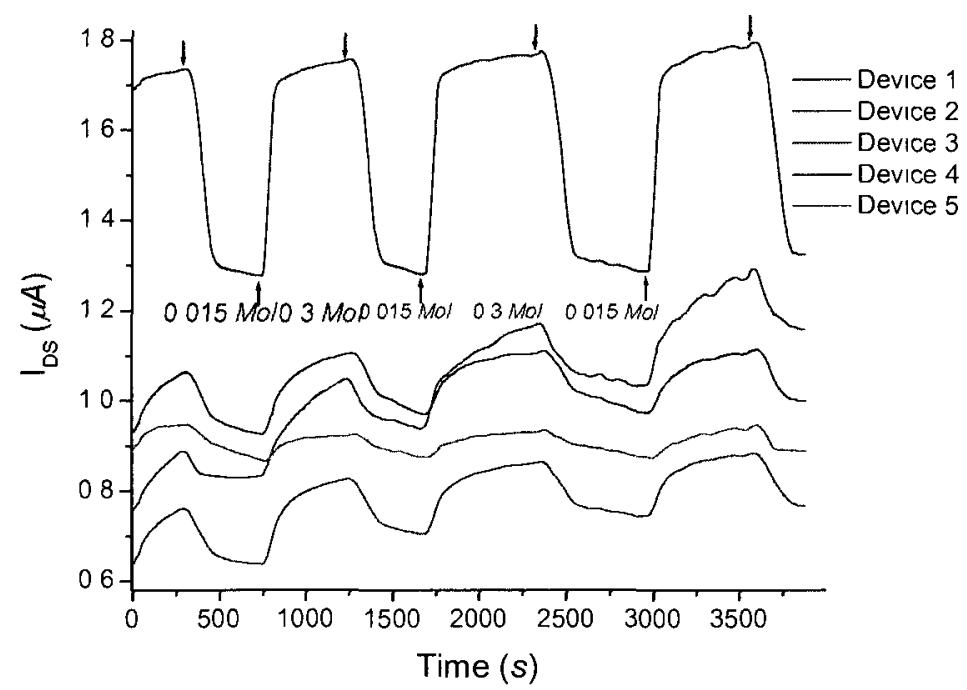

(a)

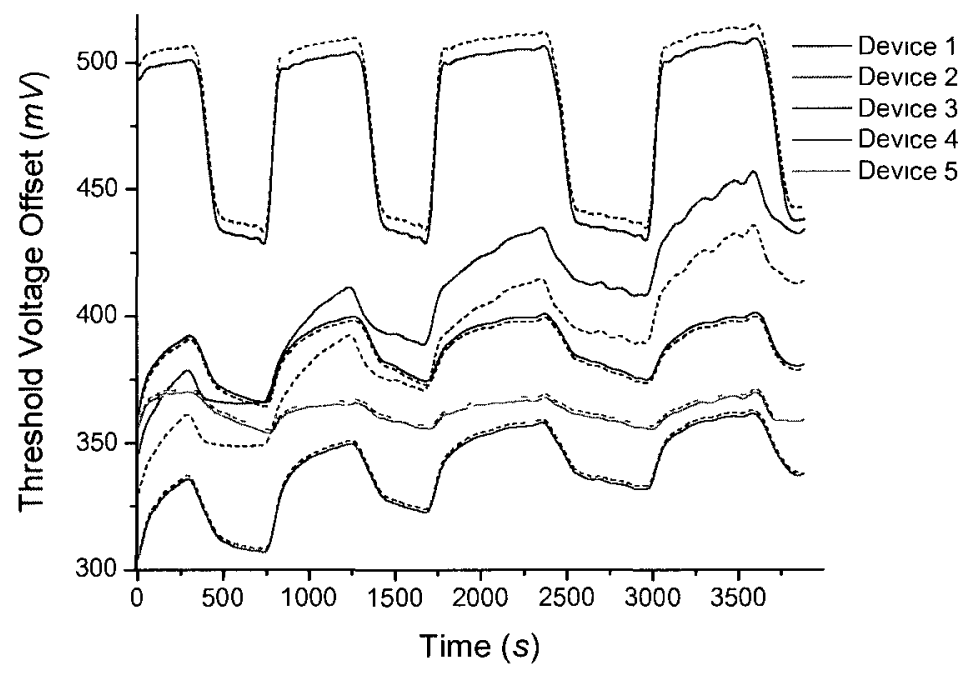

(b)

Figure 5.6 - (a) The output current, IDS vs. time after hybridization;

(b) Threshold voltage drift vs. time after hybridization. 


\begin{tabular}{|l|c|c|c|c|c|}
\hline & Device 1 & Device 2 & Device 3 & Device 4 & Device 5 \\
\hline$\Delta \mathrm{V}_{\text {T 0 1X to 2X }(\mathrm{mV})}$ & 64.0 & 34.0 & 69.6 & 28.4 & 10.0 \\
\hline$\Delta \mathrm{V}_{\mathrm{T} 2 \mathrm{X} \text { to 0 1X }(\mathrm{mV})}$ & 45.4 & 25.0 & 70.1 & 26.4 & 9.8 \\
\hline $\begin{array}{l}\text { Change in } \Delta \mathrm{V}_{\mathrm{T} 01 \mathrm{X} \text { to } 2 \mathrm{X}} \\
\text { due to hybridization }(\mathrm{mV})\end{array}$ & 7.6 & 10.3 & -2.4 & 1.9 & -18.8 \\
\hline $\begin{array}{l}\text { Change in } \Delta \mathrm{V}_{\mathrm{T} 2 \mathrm{X} \text { to 0 1X }} \\
\text { due to hybridization }(\mathrm{mV})\end{array}$ & -9.4 & 3.3 & -4.5 & 2.7 & -19.9 \\
\hline
\end{tabular}

Table 5.12 - Summary of threshold voltage shifts after hybridization.

Once again, the reference electrode work function offset due to the changes in the concentration of the electrolyte should be taken into consideration when calculating the threshold voltage shifts. However, since the work function offset is negligible comparing to the threshold voltage shift, it would not have a significant effect on the presented results.

\subsection{Discussions}

As discussed in sections 2.5 , and 4.2 , in theory, functionalization of a bioFET sensor results in a higher signal (change in the threshold voltage in response to the switch in the electrolyte) than before functionalization. This is because of the presence of the negatively charged ssDNA molecules at the sensor surface, which increases the charge density at the semiconductor-insulator interface, resulting in a larger threshold voltage in each electrolyte concentration. Therefore, the threshold voltage shift due to the transition in the electrolyte concentration will increase in response to the functionalization. 
For the same reason, the hybridization process increases the charge negativity of the DNA molecule immobilized at the surface of the bioFET, resulting in larger threshold voltage, and threshold voltage shifts due to changes in the electrolyte concentration. However, because of the increase in the charge screening by the electrolyte's positive ions, the effect of the increase in the electric field, generated by the DNA molecule, on the charges present at the channel-oxide interface is expected to be smaller than the increase in response to immobilization.

In the previous works, the threshold voltage shift in response to the functionalization has been reported from $27 \mathrm{mV}$ to $78 \mathrm{mV}$ in extreme cases. Whereas in the same works, the signal shift due to hybridization oricess has been reported to be in the range of $10 \mathrm{mV}$ to $25 \mathrm{mV}$ in extreme cases.

Such changes in the signal confirm the bonding of the ssDNA molecules to the surface, and also to the complementary ssDNA strands. If the DNA functionalization and hybridization processes are unsuccessful, these signal changes are expected to be very small, and dominated by noise [164], [185]. Sections 5.2.1 and 5.2.2 will discuss the experimental results obtained in sections 5.1.1 and 5.1.2, respectively.

\subsubsection{Gold-Chromium-Oxide Gate Devices}

The results obtained in section 5.1.1 and presented in Figure 5.7 suggest all four bioFETs in the chip under test were exceptionally sensitive to any charges present at the device interface. This was confirmed by the large sensitivity to the ionic strength variation of the electrolyte, particularly in bare gold surfaces. Comparing the obtained 
signals from results shown in the previous works confirmed this sensitivity. As predicted, this advantage is attributable to due to the larger number of binding sites available at the gold surface.

Consistency of the $\beta$ values on the bare gold surfaces before functionalization, and also before and after hybridization, in both concentrations of the electrolyte verifies all four devices had relatively similar physical characteristics at their channel and gatechannel interface.

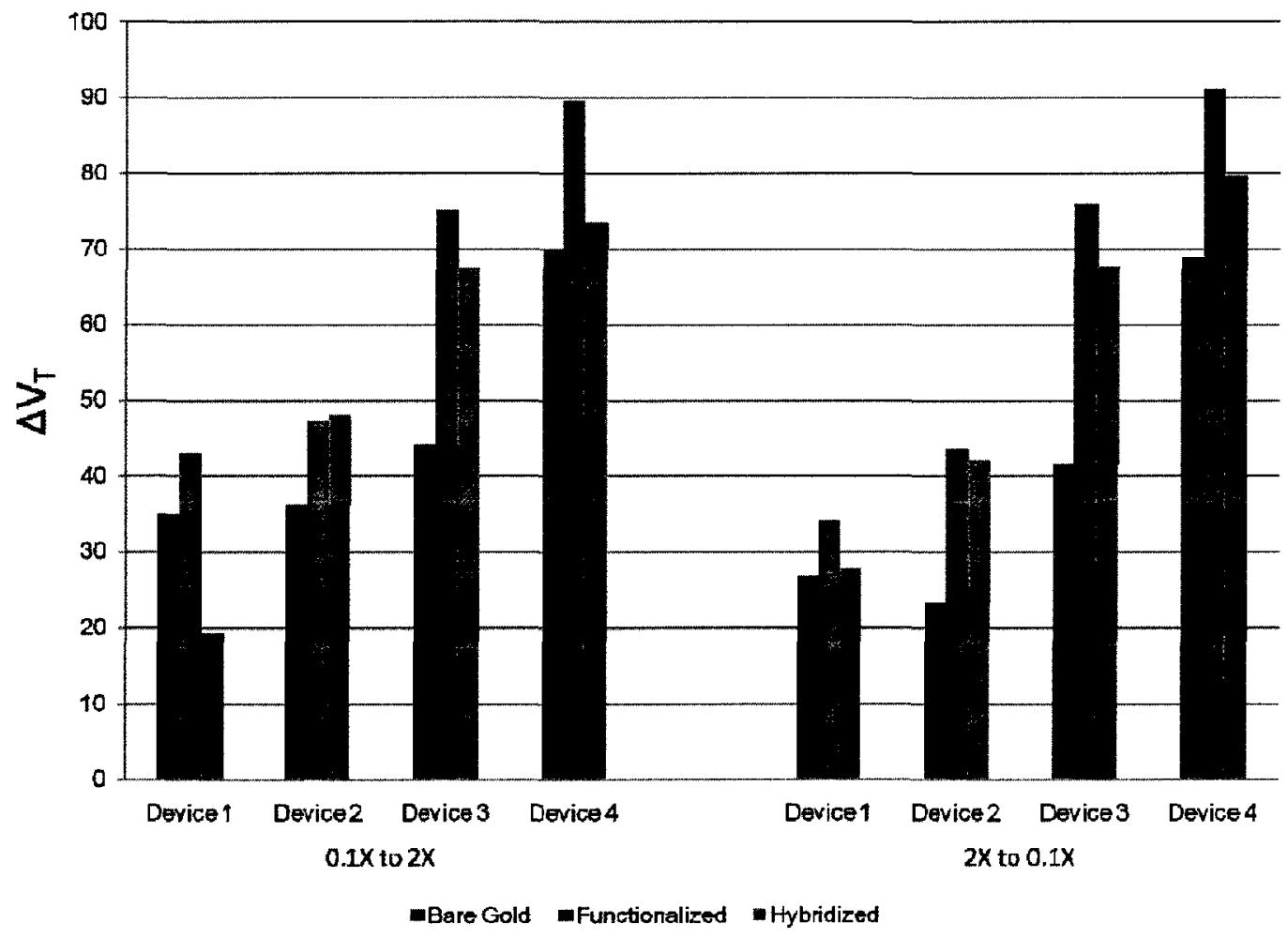

Figure 5.7 - The summary of the measurement process in bioFETs without poly, based on the calculated $\Delta \mathrm{V}_{\mathrm{T}}$ for each device 
Additionally, in parallel to the theory, the signal change in response to the DNA functionalization was much larger than the change in signal due to the hybridization process. As predicted, for most devices, the threshold voltage shift due to hybridization was smaller than the shift in response to functionalization.

It was expected that the threshold voltage shifts due to the transitions in the electrolyte concentration increase after the devices were functionalized, and also hybridized. Although this increase was seen after functionalization, a reduction was seen after hybridization. One explanation can be that the increase in charge negativity of the DNA molecule after hybridization was such that the charge screening by the electrolyte's positive ions dominated the effect of the electric field generated by DNA on the channel. Hence, it resulted in a slight reduction of the charge density at the semiconductorinsulator interface after hybridization. For this purpose, one could use peptide nucleic acid (PNA) molecules, which are complementary neutral polymers, instead of the DNA strand utilized here.

One explanation for the variation in the sensing behaviour of the devices is the lack of surface uniformity in the devices during the dry etch step of the passivation nitride layer in the post-processing. Since all the devices were not completely etched, a longer etching process expanded the exposing window of the gate, resulting in nonidentical gate surfaces. Another potential reason could be a non-uniform DNA immobilization. Even though it was assumed that the entire device surfaces would be functionalized, it is unlikely that functionalization did completely cover the surface of some devices with thiol-terminated DNA molecules. 
Moreover, the level of noise, which was primarily generated by the fluctuations of the electrolyte meniscus inside the glass ring, was much smaller than the level of the threshold voltage shifts (both in response to changes in the electrolyte or due to DNA functionalization and hybridization). Therefore, the effect of noise was ignored.

One remarkable outcome of the measurement results was reproducibility of the transitions over time under the same conditions. Even when the device measurements were repeated a day apart, the same results were obtained. Not only does the signal reproducibility represent the stability of the device, but also it shows the functionalization and hybridization processes have also been stable. This means the bonds between the thiolated ssDNA strands and the gold surface after functionalization, and also the bonds between the ssDNA strands and their complementary strands after hybridization, were not broken over time due to the presence of the electrolyte's ions attacking the bonds.

\subsubsection{Gold-Chromium-Poly-Oxide Gate Devices}

The gold coated poly gate bioFET sensors were also very sensitive to the presence of charges at their surface. Hence, they showed large response to the changes in the electrolyte concentration. Unfortunately, these devices, which were the focus of this project, showed scattered results. For example, because of the very large drifts, device 1 showed overall behaviour dissimilar to the others.

Moreover, although large, the threshold voltage shift due to the electrolyte transitions in device 3 did not change significantly in response to functionalization or hybridization. However, for the other three devices, the signal shift due to DNA 
functionalization was larger than the hybridization signal, as expected. The results summary for these devices is presented in Figure 5.8

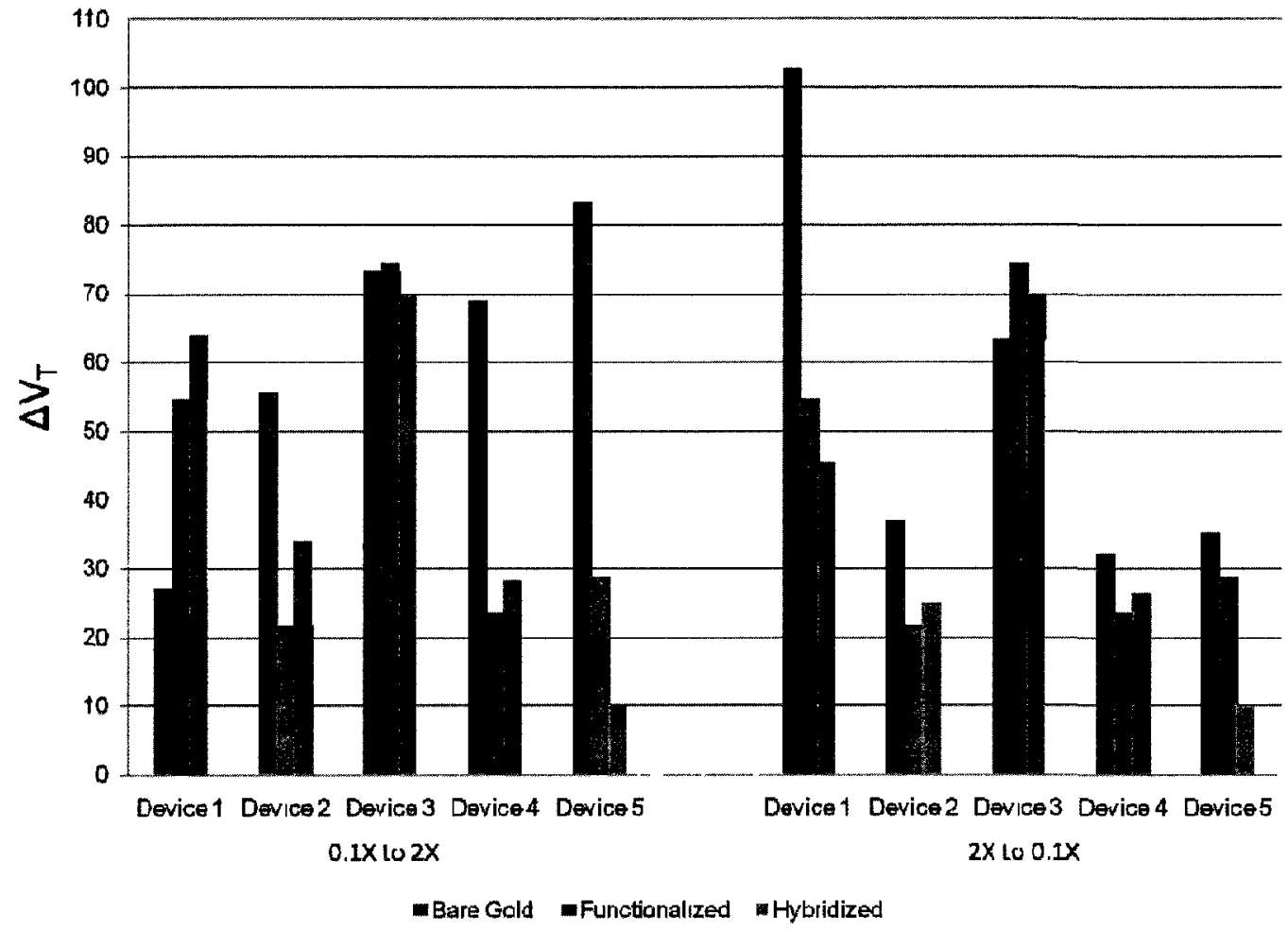

Figure 5.8 - The summary of the measurement process in gold coated poly gate bioFETs, based on the calculated $\Delta \mathrm{V}_{\mathrm{T}}$ for each device

As mentioned in sections 2.5 and 5.2.1, it was expected that the threshold voltage shift due to the electrolyte transitions increases in response to both functional and hybridization processes, but in contrast with the theory, it was reduced. This can be caused by the possibility that the hybridization process immobilized a large number of ssDNA molecules, such that the number of available binding sites at the gold surface 
reduced significantly. Therefore, the sensitivity of the gold surface to the changes of the ions in the electrolyte was reduced.

However, the increase in the signal in response to the hybridization, parallel to the theory, is seen in devices 2 and 4 (though that of the device 4 is very small). Hence, it is believed that the rise in the charge negativity of the DNA molecule resulted in larger threshold voltages. As a result, the difference between the threshold voltages of each device in the two types of electrolyte was more notable.

Nevertheless, one might believe similar to the devices discussed in section 5.2.1, the increase in the charge negativity of the DNA molecules on device 5 resulted in a larger charge screening by the electrolyte's positive ions. Therefore, the gold surface showed smaller sensitivity to the changes in the electrolyte concentration after hybridization. An explanation that device 5 behaves dissimilar to devices 2 and 4 can be possibly that the DNA hybridization was denser in this device than the other two. Therefore, it resulted in larger charge screening by the electrolyte.

The signal change due to functionalization in each device is in the $31.9 \mathrm{mV}$ to $54.6 \mathrm{mV}$, and analogously in range to the previous reported works. Additionally, the signal changes in response to hybridization, for devices 2 and 5 were $10.3 \mathrm{mV}$ and 18.8 $\mathrm{mV}$, respectively. They are also in range to the results reported based on the previous works. Similar to the devices discussed in section 5.2.1, the level of these signals is higher than the level of the signal noise. Therefore, the effect of the noise on the measurement results can also be ignored for gold coated poly gate bioFETs 
Moreover, the level of drift in each device followed the similar trend over the measurement; therefore, the effects of these drifts were predictable and they were taken into account when the threshold voltage shifts were calculated. Although subject to large drifts during the same measurements, and analogous to the poly removed gates, each individual measurement was reproducible over time. This was confirmed by measuring the signals the following day and obtaining similar behaviour. This also proves the devices were stable over time. The same affect was seen with the functionalization and hybridization processes. Even though all devices showed similar overall responses, lack of uniformity in the sensors' surface caused variation in the behaviour of the devices. 


\section{Chapter 6}

\section{Conclusion and Future Work}

In this project, arrays of gold coated bioFET sensors were fabricated on silicon $<111>$ crystal wafers and tested for their sensitivity to functionalization of thiolterminated ssDNA molecules and hybridization of their complementary DNA strand. This work focused on the bioFETs whose gates' polysilicon layers were not removed and compared them with the devices that did not have such poly layers. The device production involved standard CMOS fabrication compatible fabrication and postprocessing procedure. In both sets of biosensors, the surface material was gold. Since the number of available binding sites is very large at the gold surface, these sensors were very sensitive to the changes in the surface charges, particularly to the changes in the electrolyte concentration.

The project involved measuring multiple sensors simultaneously. Although different devices on each chip showed similar behaviour, they mostly produced adequate signals confirming that they had detected the presence of the DNA molecules through the functionalization and hybridization processes. Due to non-uniform nitride etch process during post-processing, each device had a unique surface behaviour. Despite of the initial assumptions, it is believed that the DNA immobilization and hybridization were not uniform at the device surfaces. This effect increased the irregularity in the behaviour of the devices. For most devices, analogous to the theory as well as previous works, the 
signal change in response to functionalization was larger than that due to hybridization. However, in contrast to the theory, the signal shift due to functionalization was negative in the gold coated poly gate devices. And because of the non-uniformity in the surface behaviour of the devices, the signal change after hybridization was positive in two devices and negative in another.

Direct immobilization of DNA on the bare semiconductor, especially silicon $<111>$ crystal based bioFETs, is the recent subject of many researches; however, such devices have complex surface chemical properties. Additionally, silicon-on-insulator (SOI) based bioFETs are also an appealing subject of study. They are thought to provide higher sensitivity to changes of the surface charges. The group has a potential project on this type of sensors (Silicon $<111>$ crystal based SOI bioFETs), which has been depending on the results of the work presented in this thesis.

Utilizing a control sensor in the array, which is not being immobilize, would help one monitor the changes in the devices surface, such as the observed signal drifts. Once could also make use of control FETs that are not exposed to the electrolyte and are fabricated solely to monitor the changes in the ambient conditions not related to the electrolyte, such as any temperature changes, etc. Attempting to unsuccessfully hybridize non-complementary ssDNA molecules would also enable one to confirm the success of the hybridization process.

Moreover, improving the electrolyte flow by using a closed system of microfluidic channels would have benefits such as a reduction in the transition lengths, and smaller risk of liquid overflow from the top of the glass ring which could create a 
short circuit and compromise the measurements. In the microfluidic channels, arrays of metal lines could be post-processed and laid out to act as the local reference electrode. The local electrode would be able to reduce the drifts caused by the commercial $\mathrm{Ag}-$ $\mathrm{AgCl}$ reference electrode, and also have much smaller work function offset. However, measurements using a post-processed $\mathrm{Ag}-\mathrm{AgCl}$ reference electrode are not practical, since the $\mathrm{Ag}-\mathrm{AgCl}$ electrode is not stable in electrolytes with ionic strengths far lower than saturation.

If all the devices on the chip were optimized in a way that they produced very similar signals, one could sense multiple DNA molecules and distinguish them by functionalizing a unique DNA strand to each device. Not only would this method increase the overall sensing speed, but also the detection cost would also drastically reduce as multiple samples are being detected. Attaching uncharged peptide nucleic acid probe molecules would help obtaining a larger signal due to hybridization than the functionalization signal. Moreover, if a DNA molecule could be used such that the measurements at the same time as functionalization and hybridization were feasible, once could observe the simultaneous change in signal as the surface charges are gradually modified.

Detecting presence of DNA molecules using label-free methods, especially electronic sensing through bioFETs has shown promising potentials in improving the biological recognition. Although the research in this field, including this thesis, is relatively recent, sensing the DNA hybridization with bioFETs has shown to be a successful method. Yet, there is a necessity to improve this method of detection by 
increasing the device sensitivity and uniformity of the detected signals, and increasing the detection speed. Ultimately a reduction in the effective production costs would prepare the bioFET sensors for mass manufacturing and make them available for use in any laboratory. 


\section{References}

[1] D. Landheer, G. Aers, W. R. McKinnon, M. J. Deen, and J. C. Ranuarez, "Model for the field effect from layers of biological macromolecules on the gates of metaloxide-semiconductor transistors," Journal of Applied Physics, vol. 98, no. 4, pp. 044701 - 0447015, August 2005.

[2] W.R. McKinnon and D. Landheer, "Sensitivity of a field-effect transistor in detecting DNA hybridization, calculated from the cylindrical Poisson-Boltzmann equation," Journal of Applied Physics, vol. 100, no. 5, pp. 054703 - 054703-8 , September 2006.

[3] D. Landheer, W. R. McKinnon, G. Aers, W. Jiang, and M. J. Deen, "Calculation of the Response of Field-Effect Transistors to Charged Biological Molecules," IEEE Sensors Journal, vol. 7, no. 9, pp. 1233-1242, September 2007.

[4] D. Landheer, W. R. McKinnon, W. H. Jiang, and G. Aers, "Effect of screening on the sensitivity of field-effect devices used to detect oligonucleotides," Applied Physics Letters, vol. 92, no. 25, pp. 253901 - 253901-3, June 2008.

[5] W. H. Jiang, D. Landheer, G. Lopinski, W.R. McKinnon, A. Rankin, E. GhiasBegloo, R. Griffin, N.G. Tarr, N. Tait, J. Liu, and W.N. Lennard, "Hafnium Silicate Gate Insulators in Field Effect Sensors Used to Detect DNA Hybridization," The Electrochemical Society Transaction, vol. 16, no. 11, pp. 441 - 450, November 2008.

[6] L. Stryer, J. M. Berg, and J. L. Tymoczko, Biochemistry, 5th ed. New York, NY: W.H. Freeman and Co., 2002.

[7] J. D. Watson and F. H. C. Crick, "A Structure for Deoxyribose Nucleic Acid," Nature, vol. 71, pp. 737 - 738, April 1953.

[8] E. Chargaff, "Structure and function of nucleic acids as cell constituents," Federation Proceedings, vol. 10, no. 3, pp. 654 - 659, September 1951.

[9] S. Zamenhof, G. Brawerman, and E. Chargaff, "On the desoxypentose nucleic acids from several microorganisms," Biochim Biophys Acta, vol. 9, no. 4, pp. 402 405, October 1952.

[10] R. H. Garrett and C. M. Grisham, Biochemistry. Orlando, FL: Saunders College Publishing, 1995. 
[11] M. H. F. Wilkins and J. T. Randall, "Crystallinity in sperm heads: molecular structure of nucleoprotein in vivo," Biochimica et Biophysica Acta, vol. 10, no. 1, pp. 192 - 193, January 1953.

[12] M. H. F. Wilkins, A. R. Stokes, and H. R. Wilson, "Molecular Structure of Deoxypentose Nucleic Acids," Nature, vol. 171, no. 1, pp. 738 - 740, April 1953.

[13] M. J. Heller, "DNA microarray technology: devices, systems, and applications," Annual Review of Biomedical Engineering, vol. 4, pp. 129 - 153, Mar 2002.

[14] C. A. Thomas and B. M. Dancis, "Ring stability," Journal of Molecular Biology, vol. 77, pp. $44-55,1973$.

[15] P. N. Borer, B. Dengler, I. Tinoco, Jr, and O. C. Uhlenbeck, "Stability of ribonucleic acid double-stranded helices," Journal of Molecular Biology, vol. 86, no. 4, pp. $843-853$, July 1974.

[16] J. Marmur and P. Doty, "Determination of the base composition of deoxyribonucleic acid from its thermal denaturation temperature," Journal of Molecular Biology, vol. 5, no. 1, pp. 109 - 118, July 1962.

[17] J. G. Wetmer and N. Davidson, "Kinetics of Renaturation of DNA," Journal of Molecular Biology, vol. 31, no. 1, pp. 349 - 370, 1968.

[18] R. J. Britte, D. E. Graham, and B. R. Neufeld, "Analysis of repeating DNA sequences by reassociation," Methods Enzymol, vol. 29, no. 0, pp. 363 - 418, 1974.

[19] A. Brecht, J. Ingenhoff, and G. Gauglitz, "Direct monitoring of antigen-antibodyinteractions by spectral interferometry," Sensors and Actuators B: Chemical, vol. 6, no. 1-3, pp. 96 - 100, January 1992.

[20] V. S. Lin, K. Motesharei, K. P. S. Dancil, M. J. Sailor, and M. R. Ghadiri, "A Porous Silicon-Based Optical Interferometric Biosensor," Science, vol. 278, no. 5339, pp. 840 - 843, October 1997.

[21] A. G. Frutos, J. M. Brockman, and R. M. Corn, "Reversible Protection and Reactive Patterning of Amine- and Hydroxyl-Terminated Self-Assembled Monolayers on Gold Surfaces for the Fabrication of Biopolymer Arrays," Langmuir, vol. 16, no. 5, pp. 2192 - 2197, 2000.

[22] T. L. Clare, B. H. Clare, B. M. Nichols, N. L. Abbott, and R. J. Hamers, "Functional Monolayers for Improved Resistance to Protein Adsorption: Oligo(ethylene glycol)-Modified Silicon and Diamond Surfaces," Langmuir, vol. 21, no. 44, pp. 6344 - 6355, June 2005. 
[23] A. R. Leitch, T. Schwarzacher, D. Jackson, and I. J. Leitch, In situ Hybridisation, A Practical Guide. Oxford, UK: Bios Scientific Publishers, 1994.

[24] A. M. McNicoll and M. A. Farquharson, "In situ hybridization and its diagnostic applications in pathology," The Journal of pathology, vol. 182, no. 3, pp. 250 261, July 1997.

[25] P. Gong and R. Levicky, "DNA Surface Hybridization regimes," Proceedings of National Academy of Sciences, vol. 105, no. 14, pp. 5301 - 5306, April 2008.

[26] R. K. Saiki, S. Scharf, F. Faloona, K. B. Mullis, G. T. Horn, H.A. Erlich, and N. Arnheim, "Enzymatic amplification of beta-globin genomic sequences and restriction site analysis for diagnosis of sickle cell anemia," Science, vol. 230, no. 4732, pp. 1350 - 1354, December 1985.

[27] C. R. Newton and A. Graham, PCR. Introduction to Scientific Techniques. Oxford, UK: BIOS Scientific Publishers, 1997.

[28] G. R. Taylor, PCR 2: A Practical Approach, M. J. McPherson, B. D. Hames, and G. R. Taylor, Eds. USA: Oxford University Press, 1995.

[29] W. Saenger, Principles of Nucleic Acid Structure. New York, NY: Springer, 1983.

[30] E. F. Petricoin III, J. L. Hackett, L. J. Lesko, R. K. Puri, S. I. Gutman, K. Chumakov, J. Woodcock, D. W. Feigal Jr., K. C. Zoon, and F. D. Sistare, "Medical applications of microarray technologies: a regulatory science perspective," Nature Genetics, vol. 32, pp. 474 - 479, 2002.

[31] C. Debouck and P. N. Goodfellow, "DNA microarrays in drug discovery and development," vol. 21, pp. 48 - 50, 1999.

[32] M. Yue, H. Lin, D. E. Dedrick, S. Satyanarayana, A. Majumdar, A. S. Bedekar, J. W. Jenkins, and S. Sundaram, "A 2-D microcantilever array for multiplexed biomolecular analysis," Journal of Microelectromechanical Systems, vol. 13, no. 2, pp. 290 - 299, April 2004.

[33] V. G. Cheung, M. Morley, F. Aguilar, A. Massimi, R. Kucherlapati, and G. Childs, "Making and reading microarrays," Nature Genetics, vol. 21, pp. 15 - 19, 1999.

[34] M. Schena, D. Shalon, R. W. Davis, and P. O. Brown, "Quantitative Monitoring Of Gene-Expression Patterns With A Complementary-Dna Microarray," Science, vol. 270, no. 5235, pp. 467 - 470, October 1995. 
[35] P. F. Predki, "Functional protein microarrays: ripe for discovery," Current Opinion in Chemical Biology, vol. 8, no. 1, pp. 8 - 13, February 2004.

[36] M. F. Templin, D. Stoll, M. Schrenk, P. C. Traub, C. F. Vohringer, T. O. Joos, "Protein microarray technology," Trends In Biotechnology, vol. 20, no. 4, pp. 160 166, April 2002.

[37] L. Mezzasoma, T. Bacarese-Hamilton, M. Di Cristina, R. Rossi, F. Bistoni, and A. Crisanti, "Antigen microarrays for serodiagnosis of infectious diseases," Clinical Chemistry, vol. 48, no. 1, pp. 121 - 130, January 2002.

[38] M. C. Pirrung, "How to Make a DNA Chip," Angewandte Chemie International Edition, vol. 41, no. 8, pp. 1276 - 1289, April 2002.

[39] S. Singh-Gasson, R. D. Green, Y. Yue, C. Nelson, F. Blattner, M. R. Sussman, and F. Cerrina, "Maskless fabrication of light-directed oligonucleotide microarrays using a digital micromirror array," Nature Biotechnology, vol. 17, no. 10, pp. 974 978, October 1999.

[40] M. Cretich, G. Pirri, F. Damin, I. Solinas, and M. Chiari, "A new polymeric coating for protein microarrays," Analytical Biochemistry, vol. 332, no. 1, pp. 67 - 74, September 2004.

[41] G. Pirri, M. Chiari, F. Damin, and A. Meo, "Microarray glass slides coated with block copolymer brushes obtained by reversible addition chain-transfer polymerization.," Analytical Chemistry, vol. 78, no. 9, pp. 3118 - 3124, May 2006.

[42] K. L. Cao, G. P. Anderson, F. S. Ligler, and J. Ezzel, "Detection of Yersinia pestis fraction 1 antigen with a fiber optic biosensor," Clinical Microbiology, vol. 33, no. 2, pp. 336 - 341, February 1995.

[43] B. H. Pyle, S. C. Broadway, and G. A. McFeters, "A rapid direct method for enumerating respiring enterohemorrhagic Escherichia coli O157:H7 in water," Applied Environmental Microbiology, vol. 61, pp. 2614 - 2619, 1995.

[44] M. Muratsugu, F. Ohta, Y. Miya, T. Hosokawa, S. Kurosawa, N. Kamo, and H. Ikeda, "Quartz crystal microbalance for the detection of microgram quantities of human serum albumin: relationship between the frequency change and the mass of protein adsorbed," Analytical Chemistry, vol. 65, no. 20, pp. 2933 - 2937, October 1993.

[45] G. MacBeath and S. L. Schreiber, "Printing Proteins as Microarrays for HighThroughput Function Determination," Science, vol. 289, no. 5485, pp. 1760 - 1763, September 2000. 
[46] B. B. Haab, M. J. Dunham, and P. O. Brown, "Protein microarrays for highly parallel detection and quantitation of specific proteins and antibodies in complex solutions," Genome Biology, vol. 2, no. 2, pp. 1 - 13, January 2001.

[47] R. Wiese, Y. Belosludtsev, T. Powdrill, P. Thompson, and M. Hogan, "Simultaneous Multianalyte ELISA Performed on a Microarray Platform ," Clinical Chemistry, vol. 47, no. 8, pp. 1451 - 1457, 2001.

[48] A. Halperin, A. Buhot, and E. B. Zhulina, "Sensitivity, specificity, and the hybridization isotherms of DNA chips," Biophysical Journal, vol. 86, no. 2, pp. 718 - 730, February 2004.

[49] J. Letowski, R. Brousseau, and L. Masson, "Designing better probes: effect of probe size, mismatch position and number on hybridization in DNA oligonucleotide microarrays," Journal Of Microbiological Methods, vol. 57, no. 2, pp. 269 - 278, May 2004.

[50] A. W. Peterson, R. J. Heaton, and R. M. Georgiadis, "The effect of surface probe density on DNA hybridization," Nucleic Acids Research, vol. 29, no. 24, pp. 5163 5168, December 2001.

[51] L. Ramdas, D. E. Cogdell, J. Y. Jia, E. E. Taylor, V. R. Dunmire, L. Hu, S. R. Hamilton, and W. Zhang, "Improving signal intensities for genes with lowexpression on oligonucleotide microarrays," BMC Genomics, vol. 5, no. 1, p. 35, June 2004.

[52] H. Sakai, R. Baba, K. Hashimoto, A. Fujishima, and A. Heller, "Local detection of photoelectrochemically produced $\mathrm{H} 2 \mathrm{O} 2$ with a 'wired' horseradish peroxidase microsensor," Journal of Physical Chemistry B, vol. 99, no. 31, pp. $11896-11900$, August 1995.

[53] H. Binder, "Thermodynamics of competitive surface adsorption on DNA microarrays," Journal of Physics: Condensed Matter, vol. 18, no. 18, pp. 491 - 523, May 2006.

[54] B. R. Horrocks, D. Schmidtke, A. Heller, and A. J. Bard, "SECM 24. Enzyme ultramicroelectrodes for the measurement of hydrogen peroxide at surfaces," Analytical Chemistry, vol. 65, no. 24, pp. 3605 - 3614, December 1993.

[55] G. Bhanot, Y. Louzoun, J. H. Zhu, and C. DeLisi, "The importance of thermodynamic equilibrium for high throughput gene expression arrays," Biophysical Journal, vol. 84, no. 1, pp. 124 - 135, January 2003.

[56] C. C. Chou, C. H. Chen, T. T. Lee, and K. Peck, "Optimization of probe length and the number of probes per gene for optimal microarray analysis of gene expression," 
Nucleic Acids Research, vol. 32, no. 12, July 2004.

[57] A. Pettitt and B. M. Vainrub, "Sensitive quantitative nucleic acid detection using oligonucleotide microarrays," Journal of the American Chemical Society, vol. 125, no. 26 , pp. 7798 - 7799, July 2003.

[58] J. S. Daniels and N. Pourmand, "Label-free impedance biosensors: Opportunities and challenges," Electroanalysis, vol. 19, no. 12, pp. 1239 - 1257, May 2007.

[59] B. Nagel, H. Dellweg, and L. M. Gierasch, "Glossary for chemists of terms used in biotechnology (IUPAC Recommendations 1992)," Pure and Applied Chemistry, vol. 64, no. 1, pp. $143-168,1992$.

[60] J. Wang, Analytical Electrochemistry, 3rd ed.: John Wiley and Sons-VCH, 2006.

[61] D. R. Thévenota, K. Tothb, R. A. Durstc, and G. S. Wilsond, "Electrochemical biosensors: recommended definitions and classifications," Pure and Applied Chemistry, vol. 71, no. 23, pp. 2333 - 2348, 1999.

[62] M. A. Cooper, "Label-free screening of bio-molecular interactions," Analytical and Bioanalytical Chemistry, vol. 377, pp. 834 - 842, August 2003.

[63] A. Mulchandani and A. S. Bassi, "Principles and applications of biosensors for bioprocess monitoring and control," Critical Reviews in Biotechnology, vol. 15, no. 2, pp. $105-124,1995$.

[64] D. Y. Guschin, B. K. Mobarry, D. Proudnikov, D. A. Stahl, B. E. Rittmann, and A. D. Mirzabekov, "Oligonucleotide microchips as genosensors for determinative and environmental studies in microbiology," Applied Environmental Microbiology, vol. 63, no. 6, pp. 2397 - 2402, June 1997.

[65] A. Spiro, M. Lowe, and D. Brown, "A Bead-Based Method for Multiplexed Identification and Quantitation of DNA Sequences Using Flow Cytometry," Applied Environmental Microbiology, vol. 66, no. 10, pp. 4258 - 4265, October 2000.

[66] S. Freundlieb and J. Gamer, "Chemical microarrays: a novel approach to drug discovery," New Drugs, vol. 3, pp. 54 - 60, 2002.

[67] M. A. Cooper, "Optical biosensors in drug discovery," Nature Reviews Drug Discovery, vol. 1, pp. 515 - 528, July 2002.

[68] D. C. Wijesuriya, G. P. Anderson, and F. S. Ligler, "A rapid and sensitive immunoassay for bacterial cells," in Proceedings of the 1993 ERDEC Scientific 
Conference on Chemical Defense Research, Aberdeen Proving Ground, 1993, pp. $671-677$.

[69] H. Zhu, M. Bilgin, R. Bangham, D. Hall, A. Casamayor, P. Bertone, N. Lan, R. Jansen, S. Bidlingmaier, T. Houfek, T. Mitchell, P. Miller, and R. A. Dean, "Global analysis of protein activities using proteome chips," Science, vol. 293, no. 5537, pp. 2101 - 2105 , September 2001.

[70] J. M. Nam, C. S. Thaxton, and C. A. Mirkin, "Nanoparticle-based bio-bar codes for the ultrasensitive detection of proteins," Science, vol. 301, no. 5641, pp. 1884 1886, September 2003.

[71] G. Ramsay, "DNA chips: state-of-the art," Nature Biotechnology, vol. 16, no. 1, pp. 40 - 44, January 1998.

[72] S. Singh-Gasson, R. D. Green, Y. Yue, C. Nelson, F. Blattner, M. R. Sussman, and F. Cerrina, "Maskless fabrication of light-directed oligonucleotide microarrays using a digital micromirror array," Nature Biotechnology, vol. 17, no. 10, pp. 974 978, October 1999.

[73] A. J. Baeumner, "Biosensors for environmental pollutants and food contaminants ," Analytical and Bioanalytical Chemistry, vol. 377, no. 3, pp. 434 - 445, 2003.

[74] N. Ramachandran, D. N. Larson, P. R. Stark, E. Hainsworth, and J. LaBaer, "Emerging tools for real-time label-free detection of interactions on functional protein microarrays.," FEBS Journal, vol. 272, no. 21, pp. 5412 - 5425, November 2005.

[75] H. Zhu and M. Snyder, "Protein chip technology," Current Opinion In Chemical, vol. 7, no. I, pp. 55 - 63, February 2003.

[76] M. Bally, M. Halter, J. Voros, and H.M. Grandin, "Optical microarray biosensing techniques," Surface and Interface Analysis, vol. 38, no. 11, pp. 1442 - 1458, November 2006.

[77] Biran I., Walt D. R. Epstein J. R., "Fluorescence-based nucleic acid detection and microarrays," Analytica Chimica Acta, vol. 469, no. 1, pp. 3 - 36, September 2002.

[78] E. Engvall and P. Perlmann, "Enzyme-Linked Immunosorbent Assay (Elisa) Quantitative Assay of Immunoglobulin-G," Immunochemistry, vol. 8, no. 9, pp. 871 - 875, September 1971.

[79] D. Guschin, G. Yershov, A. Zaslavsky, A. Gemmell, V. Shick, D. Proudnikov, P. Arenkov, and A. Mirzabekov, "Manual manufacturing of oligonucleotide, DNA, and protein microchips," Analytical Biochemistry, vol. 250, no. 2, pp. 203 - 211, 
August 1997.

[80] M. A. Cooper, Ed., Label-Free Biosensors: Techniques and Applications, 1st ed. Cambridge, UK: Cambridge University Press, 2009.

[81] R. Aebersold and M. Mann, "Mass spectrometry-based proteomics," Nature, vol. 422, no. 6928, pp. 198 - 207, March 2003.

[82] B. Liedberg, C. Nylander, and I. Lunstroma, "Surface plasmon resonance for gas detection and biosensing," Sensors and Actuators, vol. 4, no. 2, pp. 299 - 304, 1983.

[83] W. Knoll, "Interfaces and thin films as seen by bound electromagnetic waves," Annual Review of Physical Chemistry, vol. 49, pp. 569 - 638, 1998.

[84] J. Homola, S. S. Yee, and G. Gauglitz, "Surface plasmon resonance sensors: review," Sensors and Actuators, B: Chemical, vol. 54, no. 1, pp. 3 - 15, January 1999.

[85] J. Dostalek, H. Vaisocherova, and J. Homola, "Multichannel surface plasmon resonance biosensor with wavelength division multiplexing," Sensors and Actuators B (Chemical), vol. 108, pp. 758 - 764, July 2005.

[86] J. Homola, H. Vaisocherova, J. Dostalek, and M. Piliarik, "Multi-analyte surface plasmon resonance biosensing," Biosensors, vol. 37, no. 1, pp. 26 - 36, September 2005.

[87] B. P. Nelson, T. E. Grimsrud, M. R. Liles, R. M. Goodman, and R. M. Corn, "Surface plasmon resonance imaging measurements of DNA and RNA hybridization adsorption onto DNA microarrays," Analytical Chemistry, vol. 73, no. 1 , pp. 1 - 7, January 2001.

[88] A. G. Frutos, L. M. Smith, and R. M. Corn, "Enzymatic Ligation Reactions of DNA "Words" on Surfaces for DNA Computing," Journal of the American Chemical Society, vol. 120, no. 40, pp. 10277 - 10282, September 1998.

[89] X. L. Su and Y. Li, "Surface plasmon resonance and quartz crystal microbalance immunosensors for detection of Escherichia coli O157: H7," Transactions of the American Society of Agricultural and Biological Engineers, vol. 48, no. 1, pp. 405 $413,2005$.

[90] J. M. Brockman, B. P. Nelson, and R. M. Corn, "Surface plasmon resonance imaging measurements of ultrathin organic films," Annual Review of Physical Chemistry, vol. 51, pp. 41 - 63, October 2000. 
[91] F. Song, F. Zhou, J. Wang, N. Tao, J. Lin, R. L. Vellanoweth, Y. Morquecho, and J. Wheeler-Laidman, "Detection of oligonucleotide hybridization at femtomolar level and sequence-specific gene analysis of the Arabidopsis thaliana leaf extract with an ultrasensitive surface plasmon resonance spectrometer ," Nucleic Acids Research, vol. 30, no. 14, p. e72, July 2002.

[92] P. Gong, C. Y. Lee, L. J. Gamble, D. G. Castner, and D. W. Grainger, "Hybridization behavior of mixed DNA/alkylthiol monolayers on gold: characterization by surface plasmon resonance and $32 \mathrm{P}$ radiometric assay," Analytical Chemistry, vol. 78, no. 10, pp. 3326 - 3334, May 2006.

[93] E. Souteyrand, J. P. Cloarec, J. R. Martin, C. Wilson, I. Lawrence, S. Mikkelsen, and M. F. Lawrence, "Direct detection of the hybridization of synthetic homooligomer DNA sequences by field effect," Journal of Physical Chemistry B, vol. 101, no. 15, pp. $2980-2985,1997$.

[94] B. Schweitzer, P. Predki, and M. Snyder, "Microarrays to characterize protein interactions on a whole-proteome scale," Proteomics, vol. 3, no. 11, pp. 2190 2199, November 2003.

[95] Y. Hur, J. Han, J. Seon, Y. E. Pak, and Y. Roh, "Development of an SH-SAW sensor for the detection of DNA hybridization," Sensors and Actuators A: Physical, vol. 120, no. 2, pp. 462 - 467, May 2005.

[96] J. Fritz, M. K. Baller, H. P. Lang, H. Rothuizen, P. Vettiger, E. Meyer, H. J. Guntherodt, Ch. Gerber, and J. K. Gimzewski, "Translating Biomolecular Recognition into Nanomechanics," Science, vol. 288, no. 5464, pp. 316 - 318, April 2000 .

[97] G. Wu, R. H. Datar, K. M. Hansen, T. Thundat, R. J. Cote, and A. Majumdar, "Bioassay of prostate-specific antigen (PSA) using microcantilevers," Nature Biotechnology, vol. 19, no. 9, pp. 856 - 860, September 2001.

[98] M. Sepaniak, P. Datskos, N. Lavrik, and C. Tipple, "Microcantilever transducers : A new approach to sensor technology," Analytical Chemistry, vol. 74, no. 21, pp. 568A - 575A, November 2002.

[99] D. R. Baselt, G. U. Lee, K. M. Hansen, L. A. Chrisey, and R. J. Colton, "HighSensitivity Micromachined Biosensor," Proceedings of the IEEE, vol. 85, no. 4, pp. 672 - 680, April 1997.

[100] K. M. Hansen, H. F. Ji, G. Wu, R. Datar, R. Cote, A. Majumdar, and T. Thundat, "Cantilever-based optical deflection assay for discrimination of DNA singlenucleotide mismatches," Analytical Chemistry, vol. 73, no. 7, pp. 1567 - 1571, 
April 2001.

[101] E. B. Cooper, J. Fritz, G. Wiegand, P. Wagner, and S. R. Manalis, "Robust microfabricated field-effect sensor for monitoring molecular adsorption in liquids," Applied Physics Letters, vol. 79, no. 23, pp. 3875 - 3877, December 2001.

[102] K. W. Wee, G. Y. Kang, J. Park, J. Y. Kang, D. S. Yoon, J. H. Park, and T. S. Kim, "Novel electrical detection of label-free disease marker proteins using piezoresistive self-sensing micro-cantilevers," in Biosensors and Bioelectronics, vol. 20, Granada, April 2005, pp. 1932 - 1938.

[103] R. Marie, H. Jensenius, J. Thaysen, C. B. Christensen, and A. Boisen, "Adsorption kinetics and mechanical properties of thiol-modified DNA-oligos on gold investigated by microcantilever sensors," Ultramicroscopy, vol. 91, no. 1-4, pp. 29 - 36, May 2002.

[104] R. Marie, J. Thaysen, C. Christensen, and A. Boisen, "DNA hybridization detected by cantilever-based sensor with integrated piezoresistive readout," in MicroTAS, Malmo, Sweden, 2004, pp. 485 - 487.

[105] S. Satyanarayanaa, D. T. McCormickb, and A. Majumdar, "Parylene micro membrane capacitive sensor array for chemical and biological sensing," Sensors and Actuators B: Chemical, vol. 115, no. 1, pp. 494 - 502, May 2006.

[106] Y. Okahata, Y. Matsunobo, K. Ijiro, A. Murakami, and M. Makino, "Hybridization of nucleic acids immobilized on a quartz crystal microbalance," Journal of the American Chemical Society, vol. 114, no. 21, pp. 8299 - 8300, October 1992.

[107] J. Wang, P. E. Nielsen, M. Jiang, X. Cai, J. R. Fernandes, D. H. Grant, M. Ozsoz, A. Beglieter, and M. Mowat, "Mismatch-sensitive hybridization detection by peptide nucleic acids immobilized on a quartz crystal microbalance," Journal of Analytical Chemistry, vol. 69, pp. 5200 - 5202, 1997.

[108] A. Bardea, A. Dagan, I. Ben-Dov, B. Amit, and I. Willner, "Amplified Microgravimetric Quartz-Crystal-Microbalance Analyses of Oligonucleotide Complexes: A Route to a Tay-Sachs Biosensor Device," Chemical Communications, vol. 7, pp. 839 - 840, 1998.

[109] K. Nikura, Y. Nagata, and Y. Okahata, "Quantitative Detection of Protein Binding onto DNA Strands by using a Quartz-Crystal," Chemical Letters, vol. 10, pp. 863 $864,1996$.

[110] J. Wang, M. Jian, and E. Palecek, "Real-time monitoring of enzymatic cleavage of nucleic acids using a quartz crystal microbalance," Bioelectrochemistry and Bioenergetics, vol. 48, no. 2, pp. 477 - 480, May 1999. 
[111] H. Su, P. Williams, and M. Thompson, "Platinum anticancer drug binding to DNA detected by thickness-shear mode acoustic wave sensor," Journal of Analytical Chemistry, vol. 67, no. 5, pp. 1010 - 1013, March 1995.

[112] F. Patolsky, K. T. Ranjit, A. Lichtenstein, and I. Willner, "Dendritic amplification of DNA analysis by oligonucleotide-functionalized Au-nanoparticles," Chemical Communications, vol. 12, pp. 1025 - 1026, 2000.

[113] X. C. Zhou, S. J. O'Shea, and S. F. Y. Li, "Amplified microgravimetric gene sensor using Au nanoparticle modified oligonucleotides," Chemical Communications, vol. 11, pp. $953-954,2000$.

[114] S. Han, J. Lin, M. Satjapipat, A. Baca, and F. Zhou, "A Three-Dimensional Heterogeneous DNA Sensing Surface Formed by Attaching OligonucleotideCapped Gold Nanoparticles Onto a Gold-Coated Quartz Crystal," Chemical Communications, vol. 7, pp. 609 - 610, 2001.

[115] D. Ivnitski, I. Abdel-Hamid, P. Atanasov, and E. Wilkins, "Application of electrochemical biosensors for detection of food pathogenic bacteria," Electroanalysis, vol. 12, no. 5, pp. 317 - 325, March 2000.

[116] I. Willner, R. Blonder, and A. Dagan, "Application of photoisomerizable antigen monolayer electrodes as reversible amperometric immunosensor," Journal of the American Chemical Society, vol. 116, no. 20, pp. 9365 - 9366, October 1994.

[117] A. J. Bard, Electrochemical Methods Fundamentals and Applications, 2nd ed. New York, NY: John Wiley and Sons, 2001.

[118] D. Janger and A. Graneli, "Instrumental approach to potentiometric stripping analysis of some heavy metals," Analytical Chemistry, vol. 50, no. 13, pp. 1924 1929, November 1976.

[119] E. Katz and I. Willner, "Probing biomolecular interactions at conductive and semiconductive," Electroanalysis, vol. 15, no. 11, pp. 913 - 1245, July 2003.

[120] A. L. Ghindilis and I. N. Kurochkin, "Glucose potentiometric electrodes based on mediatorless bioelectrocatalysis. A new approach," Biosensors and Bioelectronics, vol. 9, no. 4-5, pp. 535 - 357, 1994.

[121] J. P. Cloarec, N. Deligianis, J. R. Martin, I. Lawrence, E. Souteyrand, C. Polychronakos, and M. F. Lawrence, "Immobilization of homooligonucleotide probe layers onto $\mathrm{Si} / \mathrm{SiO}(2)$ substrates: characterization by electrochemical impedance measurements and radiolabelling," Biosensors and Bioelectronics, vol. 17, no. 5, pp. 405 - 412, May 2002. 
[122] C. M. A. Brett, A. M. O. Brett, and S. H. P. Serrano, "An EIS study of DNAmodified electrodes," Electrochimica Acta, vol. 44, no. 24, pp. 4233 - 4239, July 1999.

[123] A. Bardea, F. Patolsky, A. Dagan, and I. Willner, "Sensing and amplification of oligonucleotide-DNA interactions by means of impedance spectroscopy: a route to a Tay-Sachs sensor," Chemical Communications, no. 1, pp. 21 - 22, 1999.

[124] M. Knichel, P. Heiduschka, W. Beck, G. Jung, and W. Gopel, "Utilization of a selfassembled peptide monolayer for an impedimetric immunosensor," Sensors and Actuators B: Chemical, vol. 28, no. 2, pp. 85 - 94, August 1995.

[125] F. Patolsky, M. Zayats, E. Katz, and I. Willner, "Precipitation of an insoluble product on enzyme monolayer electrodes for biosensor applications: characterization by Faradaic impedance spectroscopy, cyclic voltammetry, and microgravimetric quartz crystal microbalance analyses," Analytical Chemistry, vol. 71, no. 15, pp. $3171-3180$, August 1999.

[126] G. B. Scuster, "Long-range charge transfer in DNA: transient structural distortions control the distance dependence," American Chemical Society, vol. 33, pp. 253 260 , February 2000.

[127] B. Giese, S. Wessely, M. Spormann, U. Lindemann, E. Meggers, and M. E. Michel-Beyerle, "On the Mechanism of Long-Range Electron Transfer through DNA," Angewandte Chemie International Edition, vol. 38, pp. 996 - 998, March 1997.

[128] S. O. Kelly, N. M. Jackson, M. Hill, and J. Barton, "Long Range Electron Transfer Through DNA Films," Angewandte Chemie International Edition, vol. 38, no. 7, pp. 941 - 945, March 1999.

[129] G. Hartwich, D. J. Caruana, T. de Lumley-Woodyear, Y. Wu, C. N. Campbell, and A. Heller, "Electrochemical Study of Electron Transport through Thin DNA Films," Journal of the American Chemical Society, vol. 121, no. 46, pp. 10803 10812, November 1999.

[130] E. Palecek and M. Fojta, "Detecting DNA Hybridization and Damage," Analytical Chemistry, vol. 73, pp. 74A - 83A, 2001.

[131] J. Wang, "Towards genoelectronics: Electrochemical biosensing of DNA hybridization," Chemistry - A European Journal, vol. 5, no. 6, pp. 1681 - 1685, June 1999.

[132] S. R. Mikklesen, "Electrochemical Biosensors for DNA Sequence Detection," Electroanalysis, vol. 8, pp. 15 - 19, 1996. 
[133] E.M. Boon, D.M. Ceres, T.G. Drummond, M.G. Hill, and J.K. Barton, "Mutation detection by electrocatalysis at DNA-modified electrodes," Nature Biotechnology, vol. 18, no. 10, pp. $1096-1100$, October 2000.

[134] R. Fan, R. Karnik, M. Yue, D. Li, A. Majumdar, and P. Yang, "DNA translocation in inorganic nanotubes," Nano Letters, vol. 5, no. 9, pp. 1633 - 1637, September 2005.

[135] P.W.K. Rothemund, "Folding DNA to create nanoscale shapes and patterns," Nature, vol. 440, pp. 297 - 302, 2006.

[136] N.C. Seeman, "DNA engineering and its application to nanotechnology," Trends in Biotechnology, vol. 17, pp. 437 - 443, November 1999.

[137] Y. Cui, Q. Wei, H. Park, and C. M. Lieber, "Nanowire nanosensors for highly sensitive and selective detection of biological and chemical species," Science, vol. 293, no. 5533, pp. 1289 - 1292, August 2001.

[138] K. S. Kim, H. S. Lee, J. A. Yang, M. H. Jo, and S. K. Hahn, "The fabrication, characterization and application of aptamer-functionalized Si-nanowire FET biosensors ," Nanotechnology, vol. 20, no. 23, pp. 235501 - 235506, May 2009.

[139] G. Gruner, "Carbon nanotube transistors for biosensing applications ," Analytical and Bioanalytical Chemistry, vol. 384, pp. 322 - 335, 2006.

[140] K. Nishiguchi, N. Clement, and T. YamaguchiA. Fujiwara, "Si nanowire ionsensitive field-effect transistors with a shared floating gate," Applied Physics Letters, vol. 94, no. 16, pp. 163106 - 163106-3, April 2009.

[141] "Effects of charge screening and surface properties on signal transduction in field effect nanowire," Journal of Applied Physics, vol. 106, no. 1, pp. 014701-1 014701-8, July Y. Liu; R. W. Dutton.

[142] T. An, K. S. Kim, S. K. Hahn, and G. Lim, "Real-time, step-wise, electrical detection of protein molecules using dielectrophoretically aligned SWNT-film FET aptasensors," Lab on a Chip, vol. 10, no. 16, pp. 2052 - 2056, August 2010.

[143] Y. Tu, Y. Lin, and Z. F. Ren, "Nanoelectrode arrays based on low site density aligned carbon nanotubes," Nano Letters, vol. 3, no. 1, pp. 107 - 109, December 2003.

[144] A. Star, E. Tu, J. Niemann, J. C. P. Gabriel, C. S. Joiner, and C. Valcke, "Labelfree detection of DNA hybridization using carbon nanotube network field-effect transistors," Proceedings of the National Academy of Sciences, vol. 103, no. 4, pp. $921-926$, January 2006. 
[145] K. Balasubramanian and M. Burghard, "Detection of human adenovirus hexon antigen using carbon nanotube sensors," Analytical and Bioanalytical Chemistry, vol. 385 , no. 3 , pp. 452 - 468, June 2006.

[146] J.N. Wohlstadter, J.L. Wilbur, G.B. Sigal, H.A. Biebuyck, M.A. Billadeau, L. Dong, A.B. Fischer, S.R. Gudibande, S.H. Jameison, J.H. Kenten, J. Leginus, J.K. Leland, R.J. Massey, and S.J. Wohlstadter, "Carbon Nanotube-Based Biosensor," Advanced Materials, vol. 15, no. 14, pp. 1184 - 1187, July 2003.

[147] H. Zhu, J. D. Suter, and X. Fan, "Label-Free Optical Ring Resonator Bio/Chemical Sensors," Springer Series on Chemical Sensors and Biosensors, vol. 8, no. 3, pp. $259-279,2010$.

[148] Y. Sun Y and X. Fan, "Optical ring resonators for biochemical and chemical sensing," Analytical and Bioanalytical Chemistry, vol. 399, no. 1, pp. 205 - 211, January 2010.

[149] K. De Vos, J. Girones, T. Claes, Y. De Koninck, S. Popelka, E. Schacht, R. Baets, and P. Bienstman,, "Multiplexed Antibody Detection With an Array of Silicon-onInsulator Microring Resonators," IEEE Photonics Journal, vol. 1, no. 4, pp. 225 235, October 2009.

[150] M. Barbaro, A. Bonfiglio, and L. Raffo, "A charge-modulated FET for detection of biomolecular processes: conception, modeling, and simulation," IEEE Transactions on Electron Devices, vol. 53, no. 1, pp. 158 - 166, January 2006.

[151] K.Y. Park, K.M. Park, Y.S. Cho, S.Y. Choi, H.S. Kim, and Y.S. Bae, "Fabrication and characteristics of bioFET albumin sensor using new self-assembled monolayer," in The 13th International Conference on Solid-State Sensors, Actuators, and Microsystems, vol. 2, Seoul, Korea, 2005, pp. 1788 - 1791.

[152] M. Zayats, A. B. Kharitonov, E. Katz, A. F. Buckmann, and I. Willner, "An integrated NAD+-dependent enzyme-functionalized field-effect transistor (ENFET) system: development of a lactate biosensor," Biosensors and Bioelectronics, vol. 15, no. 11-12, pp. $671-680,2000$.

[153] T. V. Anh Dam, D. Pijanowska, W. Olthuis, and P. Bergveld, "Highly sensitive glucose sensor based on work function changes measured by an MOSFET," Analyst, vol. 128, no. 8, pp. 1062 - 1066, 2003.

[154] D. S. Kim, Y. T. Jeong, H. J. Park, J. K. Shin, P. Choi, J. H. Lee, and G. Lim, "An FET-type charge sensor for highly sensitive detection of DNA sequence," Biosensors and Bioelectronics, vol. 20, no. 1, pp. 69 - 74, July 2004. 
[155] J. Shin, D. Kim, H. Park, and G. Lim, "Detection of DNA and Protein Molecules Using an FET-Type Biosensor with Gold as a Gate Metal," Electroanalysis, vol. 16, no. 22, pp. 1912 - 1918, October 2004.

[156] L. Bousse and P. Bergveld, "Operation of chemically sensitive field-effect sensor as a function of insulator-electrolyte interface," IEEE Transactions on Electron Devices, vol. 30, no. 10, pp. 1263 - 1270, October 1983.

[157] J. Wang and A. N. Kawde, "Pencil-based renewable biosensor for label-free electrochemical detection of DNA hybridization," Analytica Chimica Acta, vol. 431, no. 2, pp. 219 - 224, March 2001.

[158] M. J. Schoning and A. Poghossian, "Recent advances in biologically sensitive field-effect transistors (BioFETs)," Analyst, vol. 127, no. 9, pp. 1137 - 1151, September 2002.

[159] M. Barbaro, A. Bonfiglio, L. Raffo, A. Alessandrini, P. Facci, and Imrich Barak, "A CMOS, Fully Integrated Sensor for Electronic Detection of DNA Hybridization," IEEE Electron Device Letters, vol. 27, no. 7, pp. 595 - 597, July 2006.

[160] S. M. Sze and K. K. Ng, Physics of Semiconductor Devices, 3rd ed. New York, NY: John Wiley and Sons, 2006.

[161] Y. Tsividis, Operation and Modeling of the MOS Transistor, 2nd ed. New York, NY: McGraw-Hill, 1999.

[162] I. P.Bergveld, "ISFET, Theory and Practice," in IEEE Sensor Conference, Toronto, 2003, pp. 1 - 26.

[163] H. S. Wong and M. H. White, "A CMOS-integrated 'ISFET-operational amplifier' chemical sensor employing differential sensing," IEEE Transactions on Electron Devices, vol. 36, no. 3, pp. 479 - 487, May 1989.

[164] D. Cahen, R. Naaman, and Z. Vager, "The Cooperative Molecular Field Effect," Advanced Functional Materials, vol. 15, no. 10, pp. 1571 - 1578, September 2005.

[165] P. Bergveld, "Thirty years of ISFETOLOGY: What happened in the past 30 years and what may happen in the next 30 years," Sensors and Biosensors B, vol. 88, no. 1, pp. 1 - 20, January 2003.

[166] M. Kosmulski, Chemical Properties of Material Surfaces, 1st ed. New York, NY: CRC Press, 2001. 
[167] P. Bergveld, "Development of an Ion-Sensitive Solid-State Device for Neurophysiological Measurements," IEEE Transactions on Biomedical Engineering, vol. 17, no. 1, pp. 70 - 71, January 1995.

[168] S. E. Lyshevski, Ed., Nano and Molecular Electronics Handbook. New York, NY: CRC Press, 2008.

[169] Y. H. Ghallab and W. Badawy, Lab-on-a-chip: Techniques, Circuits, and Biomedical Applications. Boston, MA: Artech House Publishers , 2010.

[170] W. L. Xing and J. Cheng, Frontiers in Biochip Technology. New York, NY: Springer, 2006.

[171] R. D. Rohde, H. D. Agnew, W. S. Yeo, R. C. Bailey, and J. R. Heath, "A NonOxidative Approach toward Chemically and Electrochemically Functionalizing Si(111)," Journal of the American Chemical Society, vol. 128, no. 29, pp. 9518 9525, July 2006.

[172] A. Faucheux, A. C. Gouget-Laemmel, C. H. de Villeneuve, R. Boukherroub, F. Ozanam, P. Allongue, and J. N. Chazalviel, "Well-defined carboxyl-terminated alkyl monolayers grafted onto $\mathrm{H}-\mathrm{Si}(111)$ : packing density from a combined AFM and quantitative IR study," Langmuir, vol. 22, no. 1, pp. 153 - 162, January 2006.

[173] T. Mohammed-Brahim, A. C. Salaun, and F. LeBihan, "SGFET as charge sensor: Application to chemical and biological species detection," Sensors \& Transducers Journal, vol. 90, pp. 11 - 26, April 2008.

[174] O. S. Lee and G. C. Schatz, "Interaction between DNAs on a Gold Surface," Journal of Physical Chemistry C, vol. 113, no. 36, pp. 5941 - 15947, August 2009.

[175] C. D. Bain, E. B. Troughton, Yu T. Tao, J. Evall, G. M. Whitesides, and R. G. Nuzzo, "Formation of monolayer films by the spontaneous assembly of organic thiols from solution onto gold," Journal of the American Chemical Society, vol. 111 , no. 1, pp. $321-335$, January 1989.

[176] T. M. Herne and M. J. Tarlov, "Characterization of DNA Probes Immobilized on Gold Surfaces," Journal of the American Chemical Society, vol. 119, no. 38, pp. 8916 - 8920, September 1997.

[177] D. T. Sawyer, A. Sobkowiak, and J. L. Roberts, Electrochemistry for Chemists, 2nd ed. New York, NY: John Wiley and Sons, 1995.

[178] R. J. C. Brown and M. J. T. Milton, "The microporous structure of silver/silver chloride electrodes and the implications for Harned cell operation," Accreditation and Quality Assurance, vol. 10, no. 7, pp. 352 -355, 2005. 
[179] N. G. Tarr, K. Shortt, W. Yanbin, and I. Thomson, "A sensitive, temperaturecompensated, zero-bias floating gate MOSFET dosimeter," IEEE Transactions on Nuclear Science, vol. 51, no. 3, pp. 1277 - 1282, June 2004.

[180] T. Sakata, Y. Okabe, K. Kuwabara, N.Sato, K. Ono, N. Shimoyama, K. Machida, and H. Ishii, "Surface Cleaning of Gold Structure by Annealing during Fabrication of Microelectromechanical System Devices," Japanese Journal of Applied Physics, vol. 48, pp. 026501-1 - 026501-3, February 2009.

[181] J. M. Gottfried, N Elghobashi, S. L. M. Schroeder, and K. Christmann, "Oxidation of gold by oxygen-ion sputtering," Surface Science, vol. 523, no. 1-2, pp. 89 - 102, January 2003.

[182] K. Masahiro and Sh. Noboru, "Effects of temperature, thickness and atmosphere on mixing in Au-Ti bilayer thin films," Journal of Materials Science, vol. 28, no. 18, pp. 5088 - 5091, September 1993.

[183] T. Sakata, Y. Okabe, K. Kuwabara, N. Sato, K. Kudou, K. Machida and H. Ishii, "Conformal Coating of Organic Dielectric Film on Gold Electrodes in Microelectromechanical System Devices by Electrodeposition," IEEJ Transactions on Sensors and Micromachines, vol. 127, no. 4, pp. 253 - 257, 2007.

[184] L. M. Fischer, M. Tenje, A. R. Heiskanen, N. Masuda, J. Castillo, A. Bentien, J. Emneus, M. H. Jakobsen, and A. Boisen, "Gold cleaning methods for electrochemical detection applications," Microelectronic Engineering, vol. 86, no. 4-6, pp. 1282-1285, June 2009.

[185] S. Uno, M. Iio, H. Ozawa, and K. Nakazato, "Full three-dimensional simulation of ion-sensitive field-effect transistor flatband voltage shifts due to DNA immobilization and hybridization," Japanese Journal of Applied Physics, vol. 49, no. 1, pp. 01 AG07-01 AG07-8, 2010. 


\section{Appendix I}

\section{DNA Sequence}

The DNA sequences used in this project consisted of two complementary 15-base single strands. First a 5'-GGTTGGTGTGGTTGG-3' sequence, attached to a HS- $\left(\mathrm{CH}_{2}\right)_{6}$ thiol molecule was functionalized onto the gold surface, and then the complementary 5'CCAACCACACCAACC-3' strand was hybridized to it. The DNA molecules were synthesized on a MerMADE 6 DNA synthesizer and preserved in a PBS buffer consisted of $5 \mathrm{mM}$ phosphate, $100 \mathrm{mM} \mathrm{MgCl}_{2}, 50 \mathrm{mM} \mathrm{NaCl}$ for longer stability. Functionalization and hybridization steps were followed by a washing step through flushing with the $\mathrm{MgCl}_{2}-\mathrm{NaCl}$ based PBS buffer and subsequently de-ionized water. 


\section{Appendix II}

\section{XPS Results}

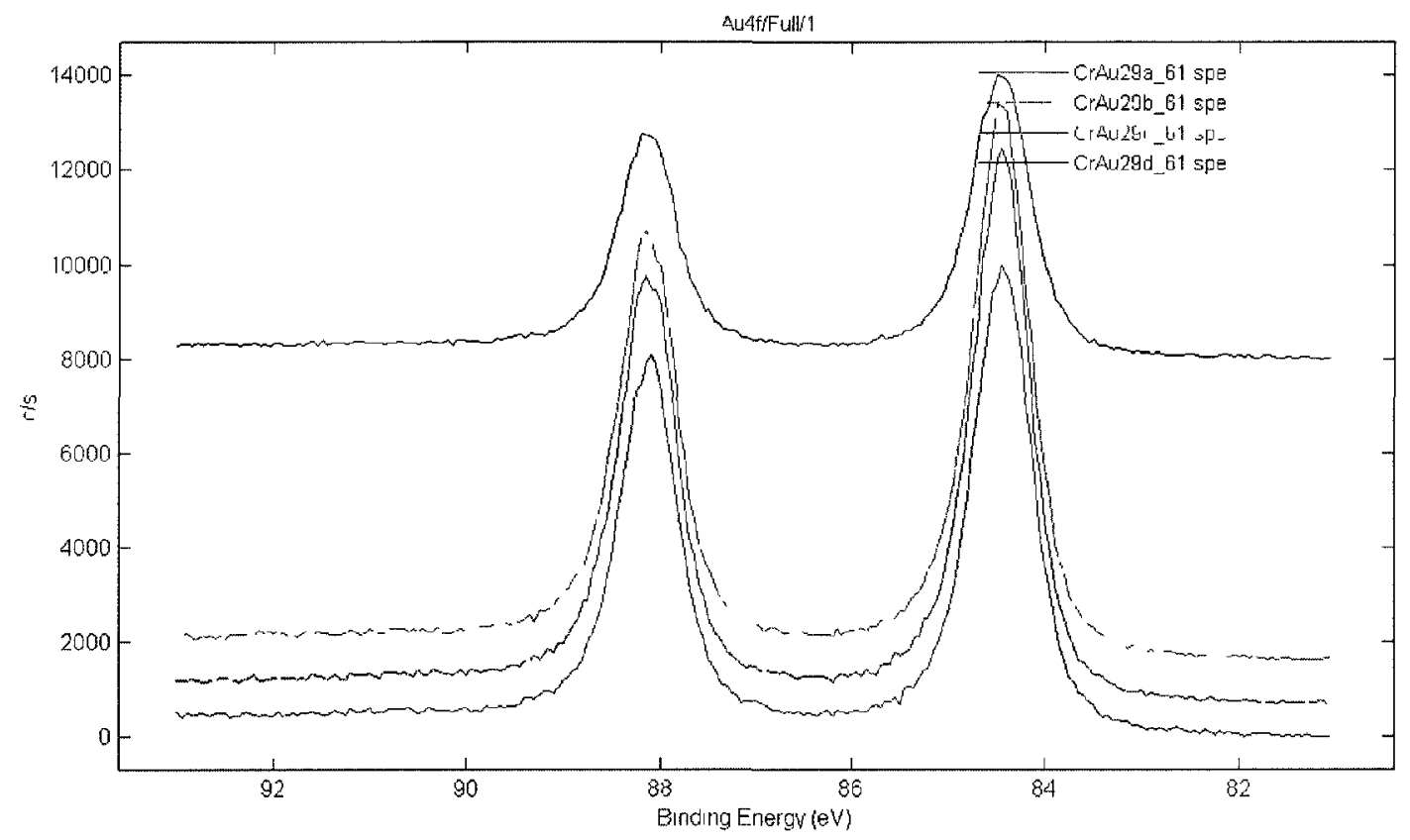

Figure II. 1 - Gold - 4 f orbital 


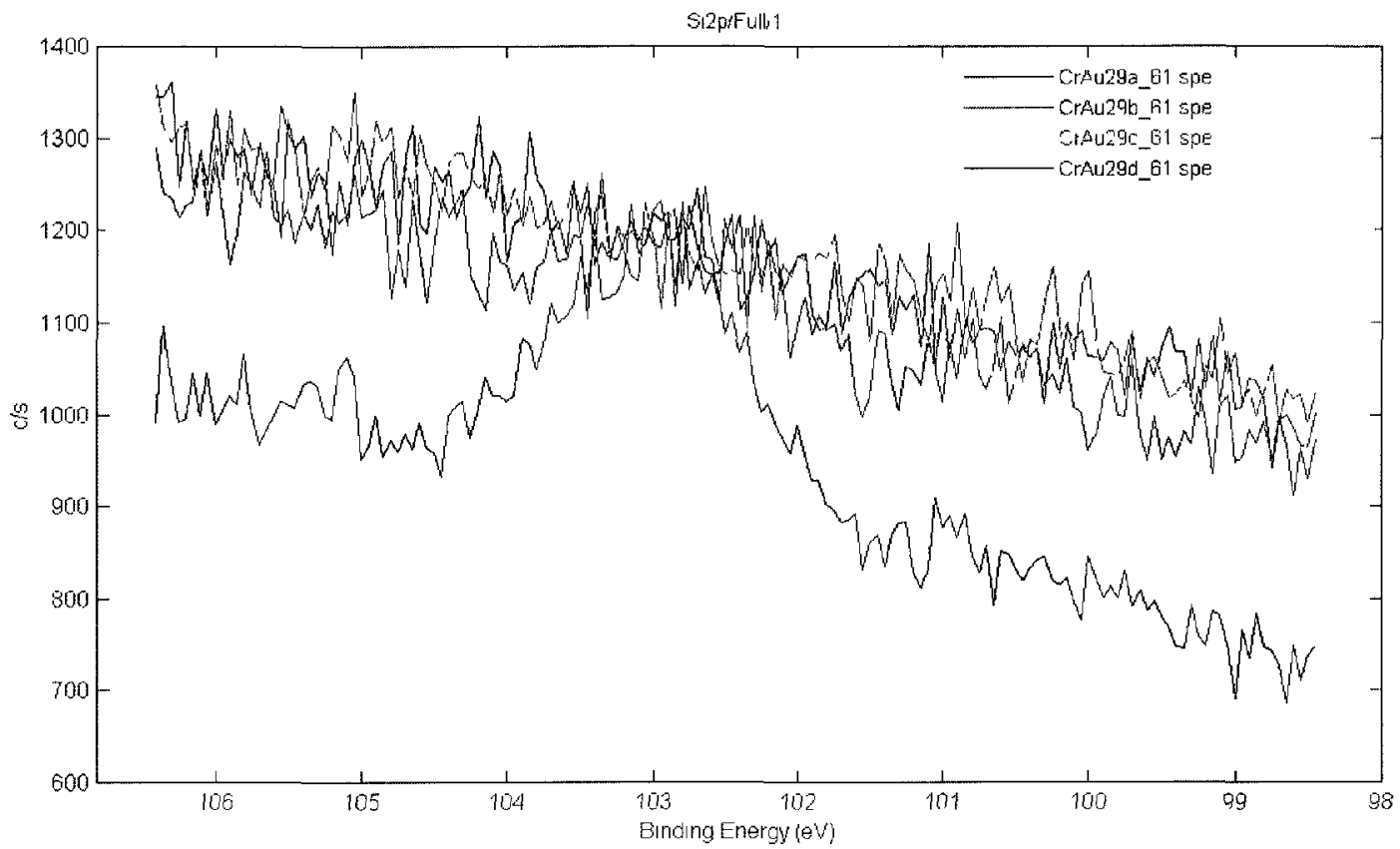

Figure II. 2 - Silicon $-2 p$ orbital

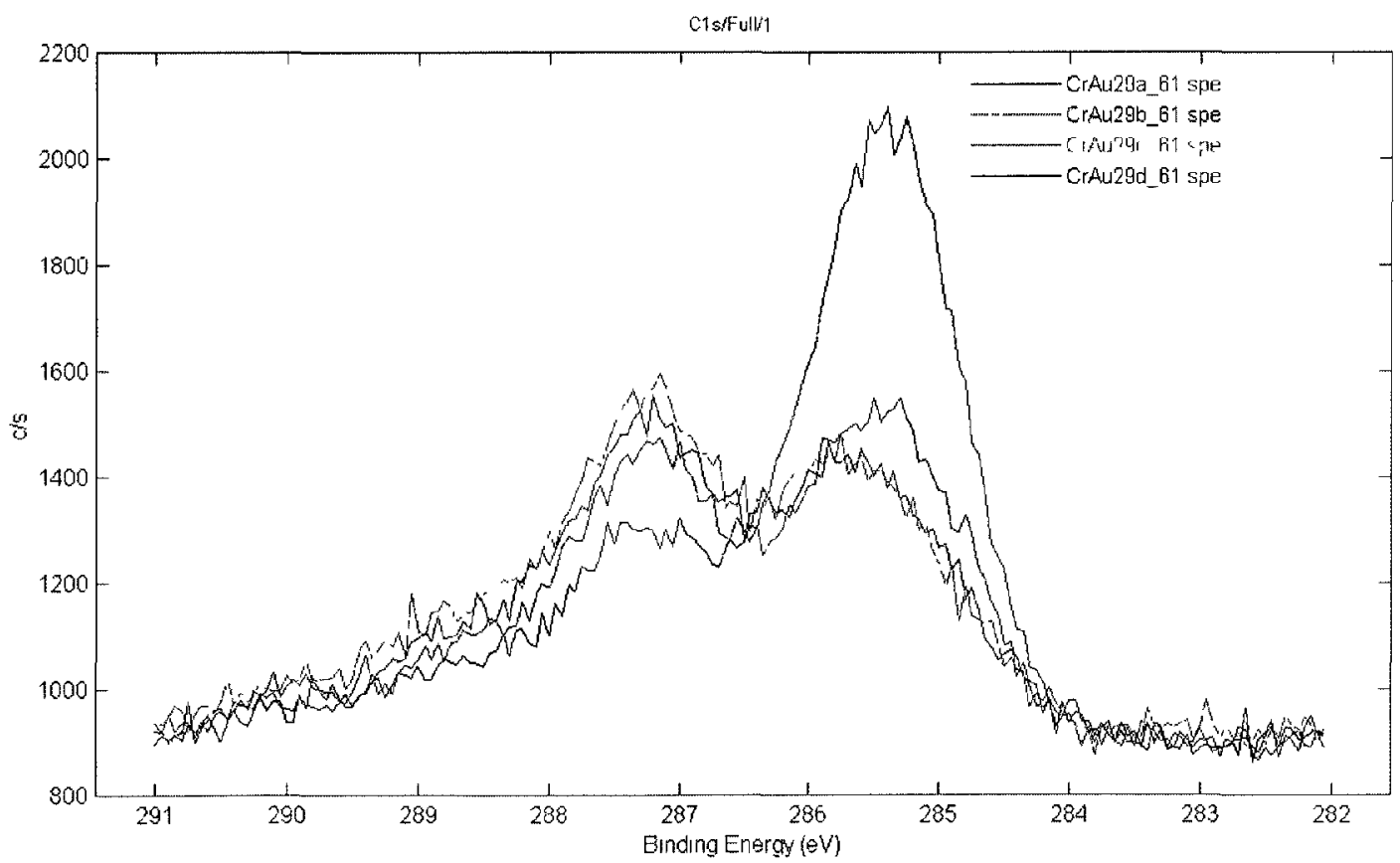

Figure II. 3 - Carbon - 1s orbital 


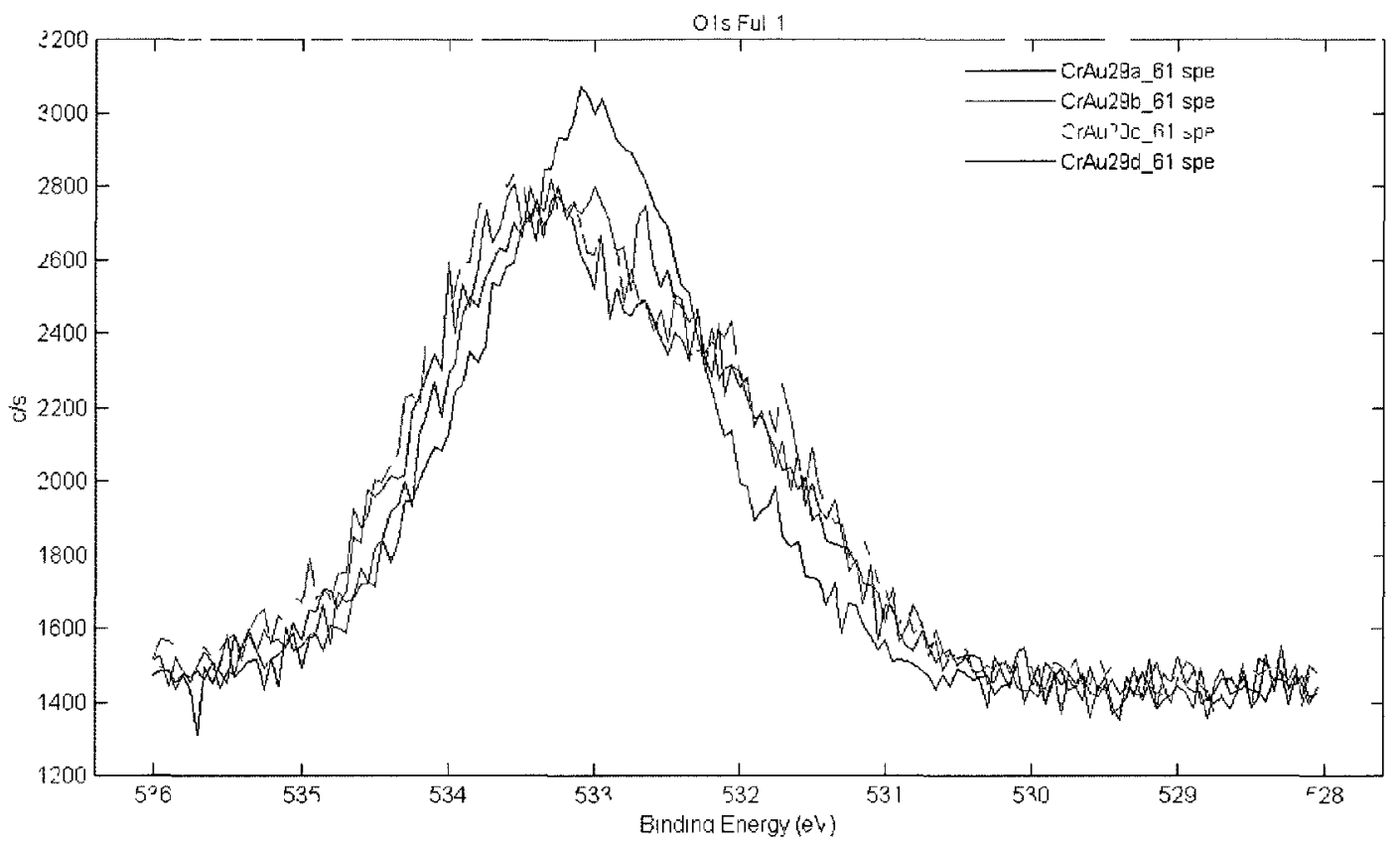

Figure II. 4 - Oxygen - 1s orbital

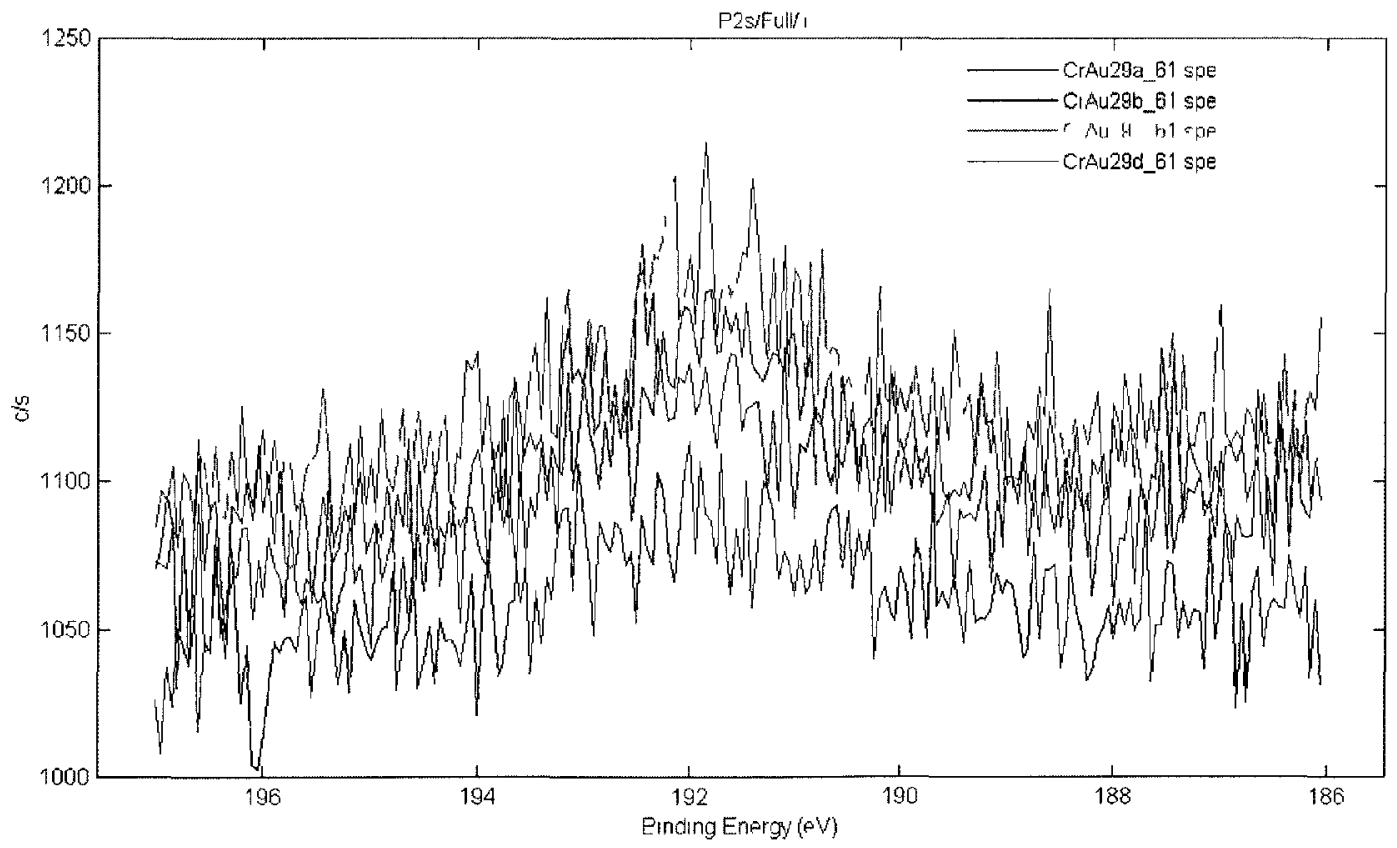

Figure II. 5 - Phosphorus - 2s orbital 


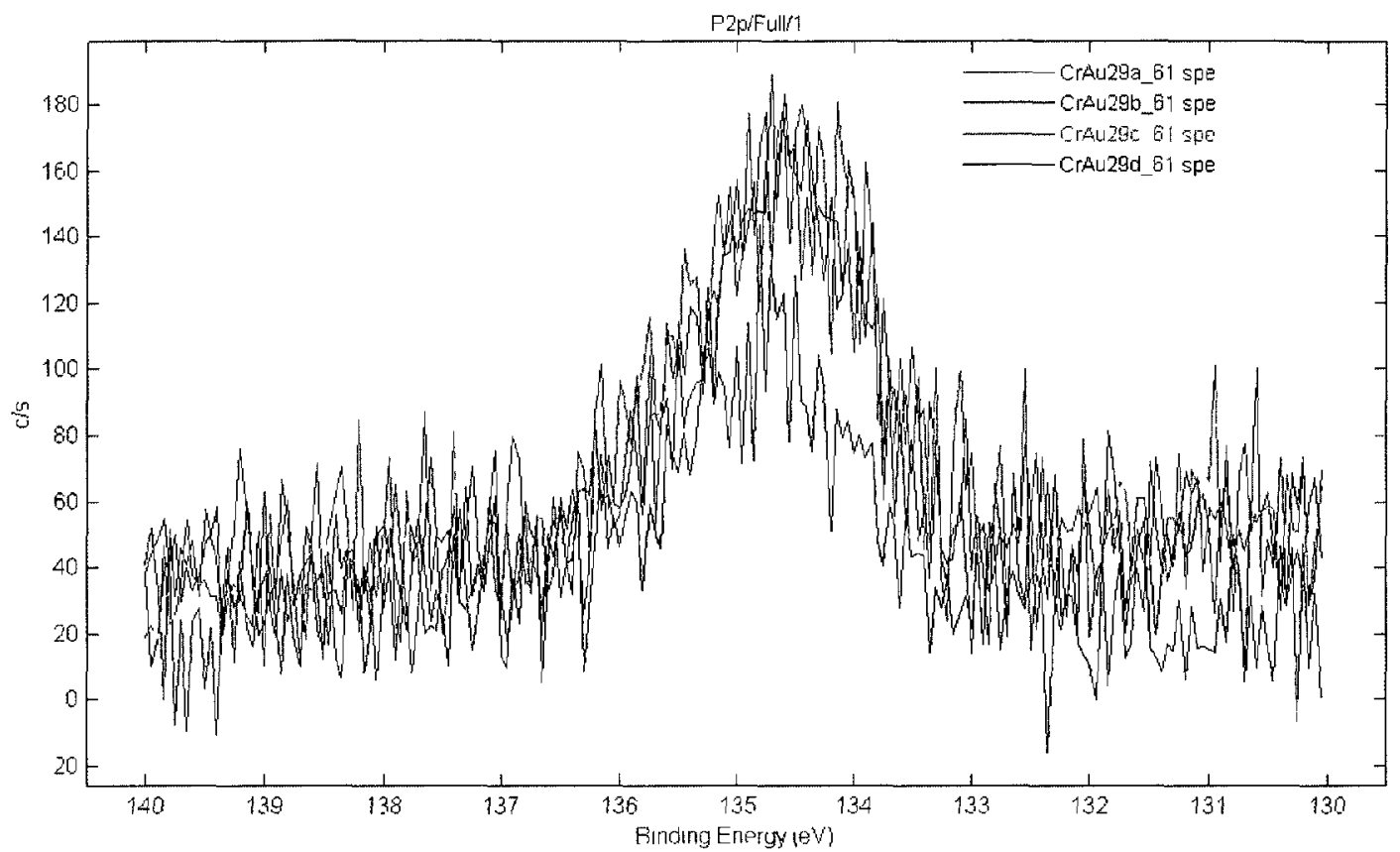

Figure II. 6 - Phosphorus $-2 p$ orbital

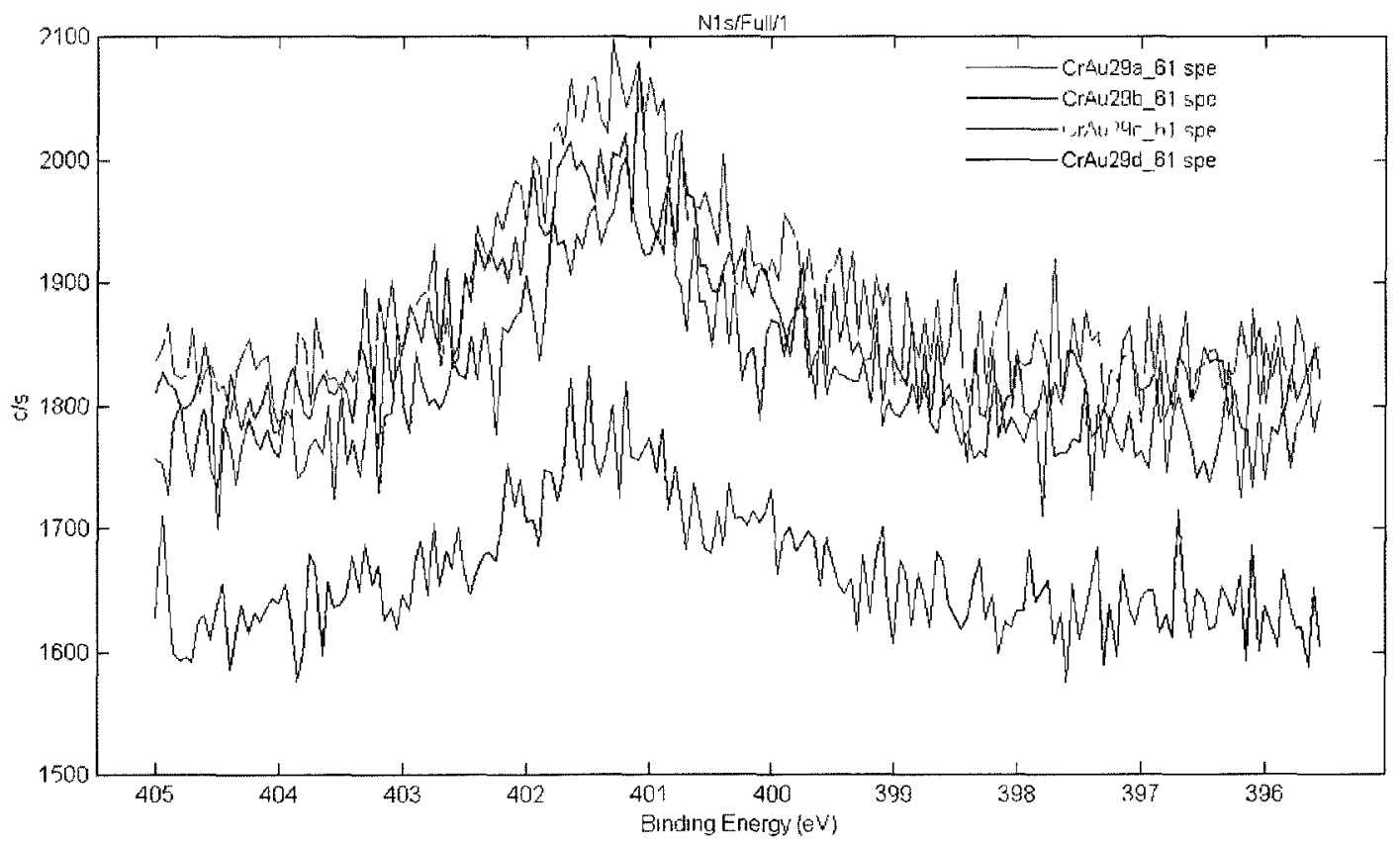

Figure II. 7 - Nitrogen - 1s orbital 


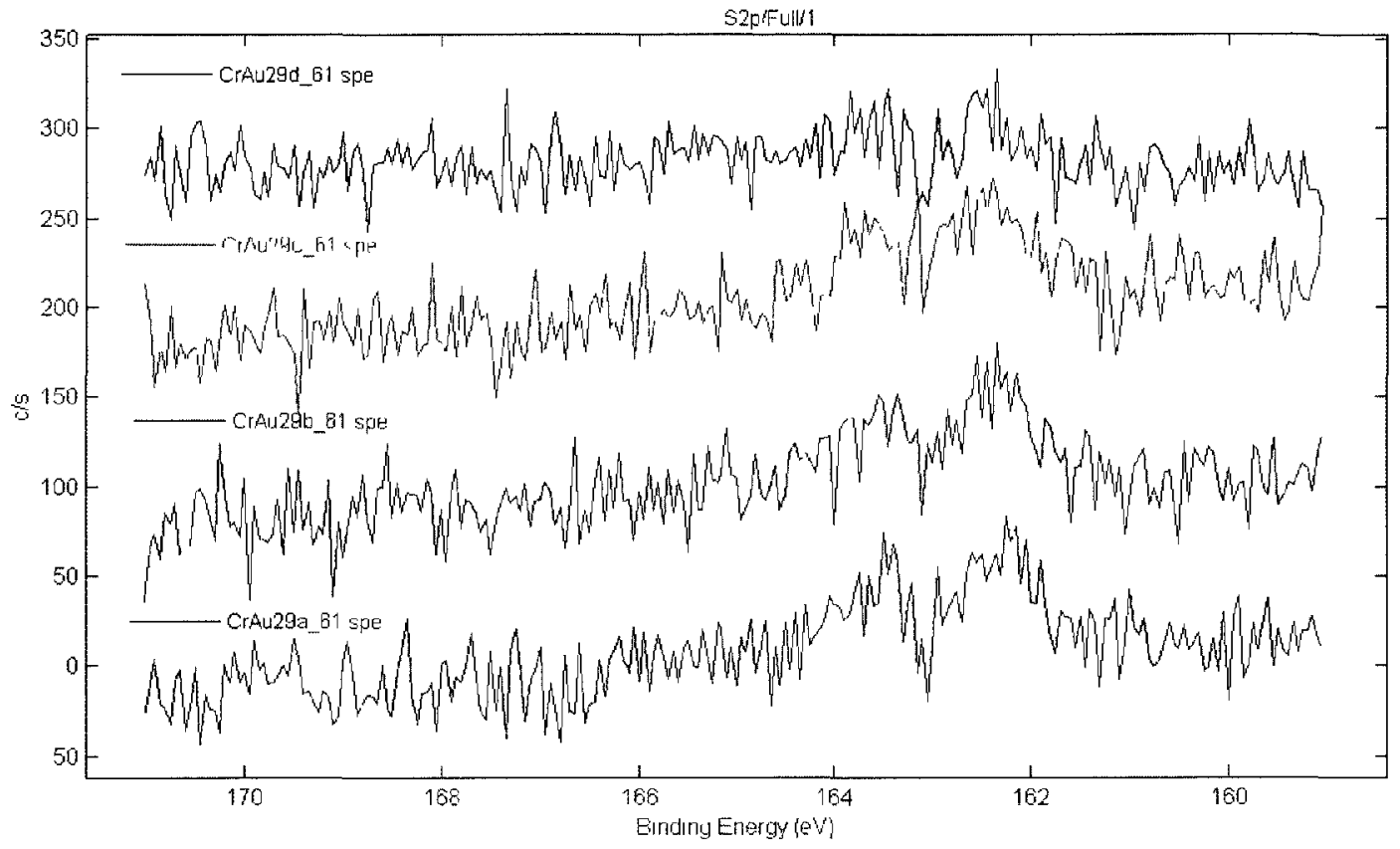

Figure II. 8 - Sulphur $-2 p$ orbital 